\title{
High-resolution multidimensional parametric estimation for nuclear magnetic resonance spectroscopy
}

\author{
by \\ Muhammad Ali Raza Anjum
}

\author{
A thesis \\ submitted to the Victoria University of Wellington \\ in fulfilment of the \\ requirements for the degree of \\ Doctor of Philosophy \\ in Electrical Engineering. \\ Victoria University of Wellington
} 2019 



\begin{abstract}
Nuclear Magnetic Resonance spectroscopy (NMR) is a powerful technique for rapid and efficient quantitation of compounds in chemical samples. NMR causes the nuclei in the molecules to resonate and various chemical arrangements appear as peaks in the Fourier spectrum of a free induction decay (FID). The spectral parameters elicited from the peaks serve as a fingerprint of the chemical components contained in the molecule. These fingerprints can be employed to understand the chemical structure.

Signal acquired from a NMR spectrometer is ideally modelled as a superposition of multiple damped complex exponentials (cisoids) in Additive White Gaussian Noise (AWGN). The number as well as the spectral parameters of the cisoids need to be estimated for characterisation of the underlying chemicals. The estimation, however, suffers from numerous difficulties in practice. These include: unknown number of cisoids, large signal length, large dynamic range, large peak density, and numerous distortions caused by experimental artefacts.

This thesis aims at the development of estimators that, in view of the above-mentioned practical features, are capable of rapid, high-resolution and apriori-information-free quantitation of NMR signals. Moreover, for the analytic evaluation of the performance of such estimators, the thesis aims to derive interpretable analytic results for the fundamental estimation theory tool for assessing the performance of an unbiased estimator: the Cramer Rao Lower Bound (CRLB). By such results, we mean those that analytically allow the determination, in terms of the CRLB, of the impact of the free model parameters on the estimator performance.

For the CRLB, we report analytic expressions on the variance of unbiased parameter estimates of damping factors, frequencies and complex
\end{abstract}


amplitudes of an arbitrary number of damped cisoids embedded in AWGN. In addition to the CRLB, analytic expressions for the determinant and the condition number of the associated Fisher Information Matrix (FIM) are also reported. Further results, in similar order, are reported for two special cases of the damped cisosid model: the Magnetic Resonance Relaxometry model and the amplitude-only model (employed in quantitative NMR qNMR). Some auxiliary results for the above-mentioned models are also presented, i.e., on the multiplicity of the eigenvalues and the factorisation of the characteristic polynomial associated with their respective FIMs.

These results have not been previously reported. The reported theoretical results successfully account for various physical and chemical phenomena observed in experimental NMR data, and quantify their impact on the accuracy of an unbiased estimator as a function of both model and experimental parameters, e.g., influence of prior knowledge, peak multiplicity, multiplet symmetry, solvent peak, carbon satellites, etc.

For rapid, high-resolution and apriori-information-free quantitation of NMR signals, a sub-band Steiglitz-McBride algorithm is reported. The developed algorithm directly converts the time-domain FID data into a table of estimated amplitudes, phases, frequencies and damping factors, without requiring any previous knowledge or pre-processing. A 2D subband Steiglitz-McBride algorithm, for the quantitation of 2D NMR data in a similar manner, is also reported. The performance of the developed algorithms is validated by their application to experimental data, which manifests that they outperform the state-of-the-art in terms of speed, resolution and apriori-information-free operation. 


\section{Acknowledgements}

Special thanks to my advisers and my family for their patience, encouragement and support. 


\section{Contents}

1 Introduction $\quad 5$

1.1 Research motivation ... . . . . . . . . . . 5

1.2 Problem statement . . . . . . . . . . . . 8

1.3 Organisation of the thesis . . . . . . . . . . 9

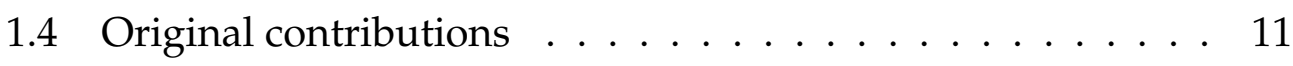

1.5 List of publications . . . . . . . . . . . . . 13

1.6 Notations . . . . . . . . . . . . . . . . . . . . . . 14

2 Introduction to NMR $\quad 15$

2.1 Background and significance . . . . . . . . . . . 15

2.1 .1 Advantages of NMR . . . . . . . . . . . . 17

2.2 NMR physics . . . . . . . . . . . . . . . . . 18

2.2.1 Spin and the spin angular momentum . . . . . . . 18

2.2.2 Spin relaxation . . . . . . . . . . . . . 21

2.2.3 Spin-Lattice relaxation time $\left(T_{1}\right) \ldots \ldots . \ldots 21$

2.2.4 Spin-Spin relaxation time $\left(T_{2}\right) \ldots \ldots . \ldots 22$

2.2.5 The rotating frame of reference . . . . . . . . . 22

2.2.6 Bloch equations of magnetisation motion . . . . . . 22

2.3 The 1D-NMR experiment . . . . . . . . . . . 26

2.3.1 The FID signal model . . . . . . . . . . . . 27

2.3.2 Practical features . . . . . . . . . . . . . 28

2.3.3 Motivation for work carried in Chapter 5: Quantitation algorithm for 1D-NMR signals . . . . . . . . . 29 
2.3.4 Motivation for work carried in Chapter 3: The CRLB 29

2.4 The 2D-NMR experiment . . . . . . . . . . . . 30

2.4.1 Motivation for work carried in Chapter 7: Quantitation algorithm for 2D-NMR signals . . . . . . . 30

2.5 Further related NMR concepts . . . . . . . . . . . . . . . 31

2.5.1 Chemical shift . . . . . . . . . . . . 31

2.5 .2 The ppm scale . . . . . . . . . . . . 32

2.5 .3 Spin-Spin coupling . . . . . . . . . . . 33

2.5 .4 The $n+1$ rule . . . . . . . . . . . . . 33

2.5.5 Carbon satellites .............. 33

3 Parametric estimation in NMR 35

3.1 Background . . . . . . . . . . . . . . 35

3.2 Significance of the models considered . . . . . . . . . . . 40

3.2.1 The MRS model . . . . . . . . . . . . . . . . . . . . 40

3.2.2 The MRR model . . . . . . . . . . . . . . . 41

3.2.3 The MRS model with previous knowledge . . . . . . 41

3.3 Problem motivation . . . . . . . . . . . . . . . . 42

3.3 .1 The CRLB . . . . . . . . . . . . . . . 42

3.3.2 The determinant, trace, and minimum and maximum eigenvalues ................. 43

3.3.3 The condition number . . . . . . . . . . . . 44

3.4 Problem statement . . . . . . . . . . . . . . . . . 44

3.5 The Leverrier-Faddeev method . . . . . . . . . . . . . . 45

3.5.1 Leverrier's method . . . . . . . . . . . . . . . 45

3.5.2 Modifications proposed by Faddeev . . . . . . . . 46

3.5.3 Computation of matrix inverse . . . . . . . . . . 47

3.5.4 Computation of determinant . . . . . . . . . . 47

3.5.5 Computation of eigenvalues . . . . . . . . . . 48

3.6 The Lobachevsky-Graeffe method . . . . . . . . . . . . . . 49

3.6.1 Basic principle. . . . . . . . . . . . . . . . 49 
3.6.2 The root separation process . . . . . . . . . 50

3.6.3 Hua's theorem . . . . . . . . . . . . . 52

3.6.4 Computation of largest eigenvalue . . . . . . . . . 53

3.6.5 Computation of smallest eigenvalue . . . . . . . . 54

3.6.6 Computation of condition number . . . . . . . . . 54

3.7 Results ....................... 55

3.7.1 The MRS model . . . . . . . . . . . . . . . 55

3.7.2 The MRR model . . . . . . . . . . . . . . 66

3.7.3 The MRS model with previous knowledge . . . . . 72

3.8 Application . . . . . . . . . . . . . . . . 78

3.8.1 Impact of model type . . . . . . . . . . . . . . . 82

3.8.2 Impact of multiplicity . . . . . . . . . . . 82

3.8 .3 Impact of symmetry . . . . . . . . . . . . 83

3.8.4 Impact of solvent peak . . . . . . . . . . . . . . . . 84

3.8.5 Impact of carbon satellites . . . . . . . . . . . 85

3.8.6 Impact of second-order effects . . . . . . . . . . 87

3.8.7 Impact of baseline distortion . . . . . . . . . . 88

3.8.8 Impact of FID truncation . . . . . . . . . . . . . 88

3.9 Conclusion . . . . . . . . . . . . . . . . . . . . 89

4 Quantitation methods for 1D-NMR signals $\quad 91$

4.1 Time-domain methods . . . . . . . . . . . . . . . . . . 91

4.1.1 Interactive methods . . . . . . . . . . . . . . 91

4.1.2 Black-box methods . . . . . . . . . . . . . . 95

4.2 Frequency-domain methods . . . . . . . . . . . . . . 99

4.2.1 Peak-area integration methods . . . . . . . . . . . 99

4.2.2 Model-fitting methods . . . . . . . . . . . . . . . 101

4.3 Time-domain vs frequency-domain methods . . . . . . . . . 103

4.4 Combined methods . . . . . . . . . . . . . . . . . . . . . . . . 104

4.4.1 Time-domain frequency-domain (TDFD) fitting . . . 104

4.4.2 SVD-based frequency-selective methods . . . . . . . 104 
4.4.3 Method based on maximum likelihood and Bayesian analysis . . . . . . . . . . . 105

4.4.4 Filter diagonalization method . . . . . . . . . 105

5 Quantitation algorithm for 1D-NMR signals 107

5.1 Introduction . . . . . . . . . . . . . . . . . . 107

5.2 Problem formulation . . . . . . . . . . . . . . . . 110

5.2 .1 Signal model . . . . . . . . . . . . . . . 110

5.2.2 Least square formulation . . . . . . . . . . . 111

5.2 .3 ARMA representation . . . . . . . . . . . 111

5.3 Steiglitz-McBride algorithm . . . . . . . . . . . . . . . 111

5.4 Model order selection . . . . . . . . . . . . . . . . . . . . . 112

5.5 Adaptive subband decomposition . . . . . . . . . . . 113

5.6 Algorithm . . . . . . . . . . . . . . . . . . . 114

5.7 Experimental results . . . . . . . . . . . . . . . . . . 115

5.7.1 Acquisition/processing parameters . . . . . . . 115

5.7.2 Comparison with SMA . . . . . . . . . . . 115

5.7.3 Comparison with LCModel . . . . . . . . . . . . 116

5.7.4 Comparison with CRAFT . . . . . . . . . . . 120

5.8 Conclusion . . . . . . . . . . . . . . . . . 120

6 Quantitation methods for 2D-NMR signals 123

6.1 Basic 2D NMR experiment . . . . . . . . . . . . . . . . 123

6.2 Types of 2D-NMR experiments . . . . . . . . . . . . 124

6.2.1 Homonuclear 2D-NMR experiments . . . . . . . . . . 124

6.2.2 Heteronuclear 2D-NMR experiments . . . . . . . 126

6.2.3 Spatial 2D-NMR experiments . . . . . . . . . . 126

6.3 Applications of 2D NMR . . . . . . . . . . . . . . . . 128

6.4 Practical issues of 2D-NMR . . . . . . . . . . . . . 128

6.4.1 Limitations imposed by the acquisition time . . . . 128

6.4.2 Limitations imposed by FFT . . . . . . . . . . . . . . 129

6.5 Addressing the limitations . . . . . . . . . . . . . . . . . 130 
6.5.1 Pre-acquisition methods . . . . . . . . . . . . 130

6.5.2 Post-acquisition methods . . . . . . . . . . . 130

6.6 Non-uniform sampling methods . . . . . . . . . . . . . 131

6.6.1 Deterministic sampling methods . . . . . . . . . . . 131

6.6.2 Irregular sampling methods . . . . . . . . . . . . 132

6.7 Uniform sampling methods . . . . . . . . . . . . . . . 132

7 Quantitation algorithm for 2D-NMR signals 135

7.1 Introduction . . . . . . . . . . . . . 136

7.2 Problem formulation . . . . . . . . . . . . . 138

7.2.1 The 2D signal model . . . . . . . . . . . . . . . . 139

7.2.2 Least square formulation . . . . . . . . . . . . . . . . . . . . . . . . . . . .

7.2 .3 ARMA modelling . . . . . . . . . . . . . . 140

7.3 2D Steiglitz-McBride algorithm . . . . . . . . . . . . . . . . . . . . . . . . . . . . . . . . . .

7.4 Model order selection . . . . . . . . . . . . . . . . . . . 142

7.5 Adaptive subband decomposition . . . . . . . . . . . 143

7.6 Algorithm . . . . . . . . . . . . . . . . 144

7.7 Experimental results . . . . . . . . . . . . . 145

7.8 Conclusion . . . . . . . . . . . . . . . 147

8 Conclusions and future work $\quad 151$

8.1 Conclusions . . . . . . . . . . . . . . . 151

8.1.1 Analytical evaluation of the CRLB . . . . . . . . . 152

8.1.2 Quantitation algorithm for 1D-NMR data . . . . . . 152

8.1.3 Quantitation algorithm for 2D-NMR data . . . . . . 153

8.2 Future work . . . . . . . . . . . . . . . . 153 


\section{List of Figures}

1.1 NMR data processing cycle [240]. . . . . . . . . . . 6

2.1 A schematic representation of NMR as partially overlapping areas representing the three main applications [77]. . . 18

2.2 Physical basis of NMR [36]. . . . . . . . . . . . . . . . 20

2.3 Real and imaginary parts of FID of ethanol recorded on Spinsolve Benchtop NMR (Magritek; Wellington, New Zealand). 27

2.4 Illustration of shielding effect responsible for chemical shift [138]. . . . . . . . . . . . . . . . . . 32

3.1 Amplitude interaction term (3.61) as a function of frequency separation $\omega_{12}$ and $k\left(\alpha_{1}=k \alpha_{2}\right)$ for the MRS model. . . . . . 61

3.2 Damping factor and frequency interaction term (3.62) as a function of $\omega_{12}$ and $k\left(\alpha_{1}=k \alpha_{2}\right)$ for the MRS model. . . . . . 62

3.3 Logarithmic plot of amplitude and damping factor and frequency interaction terms (3.61) and (3.62) as a function of $k$ $\left(\alpha_{1}=k \alpha_{2}\right)$ for the MRS model. . . . . . . . . . . . . 62

3.4 Logarithmic magnitude plot of amplitude and damping factor and frequency interaction terms (3.61) and (3.62) as a function of $\omega_{12}$ for the MRS model. . . . . . . . . . . . . 63

3.5 Comparison of amplitude bound (3.59) and the damping factor and frequency bound (3.60) for two damped cisoids for the MRS model. . . . . . . . . . . . . . . . . . . . 63 
3.6 Amplitude interaction term (3.86) as a function of $\alpha_{1}$ and $\alpha_{2}$ for the MRR model. . . . . . . . . . . . . . . . . . . . 69

3.7 Damping factor and frequency interaction term (3.87) as a function of $\alpha_{1}$ and $\alpha_{2}$ for the MRR model. . . . . . . . . . 69

3.8 Logarithmic plot of amplitude and damping factor and frequency interaction terms (3.86) and (3.87) as a function of $k$ with $\alpha_{1}=k \alpha_{2}$ for the MRR model. . . . . . . . . . . 70

3.9 Comparison of amplitude bound (3.84) and the damping factor bound (3.85) for two exponentials for the MRR model. 70

3.10 Amplitude interaction term (3.103) as a function of $\omega_{12}$ and $k\left(\alpha_{1}=k \alpha_{2}\right)$ for the amplitude-only model. . . . . . . . 75

3.11 Logarithmic plot of the amplitude interaction term (3.103) of the amplitude-only model and the amplitude and damping factor and frequency interaction terms (3.61) and (3.62) of the MRS model as a function of $k$ with $\alpha_{1}=k \alpha_{2} . \ldots$. . . 75

3.12 Logarithmic magnitude plot of the amplitude interaction term (3.103) of the amplitude-only model and the amplitude and damping factor and frequency interaction terms (3.61) and (3.62) of the MRS model as a function of $\omega_{12}$. . . 76

3.13 Chemical structure of pentanoic acid [12] . . . . . . . . . 76

$3.14{ }^{1} \mathrm{H}$ NMR spectrum of pentanoic acid. . . . . . . . . . . . 79

3.15 Carbon satellites in the vicinity of the triplet (e) of the pentanoic acid molecule. . . . . . . . . . . . . . . 86

5.1 Illustration of the employed filterbank structure and the decomposition stages [58]. . . . . . . . . . . . . . 113

5.2 Comparison of regenerated FID spectra of ethanol to the original one. At this scale, no difference is visible between the true ethanol spectrum and that reconstructed using the proposed algorithm. . . . . . . . . . . . . 116 
5.3 Comparison of the original and estimated ${ }^{1} \mathrm{H}$ spectrum of the lactose signal. Inset displays the zoomed view of the smallest peak captured by the proposed approach. This peak is as almost 35,000 times smaller than the largest peak in the signal. The proposed algorithm took just 5.7 seconds to capture all 149 peaks in the signal. The mean squared error achieved was $2.5 \times 10^{-5}$.

5.4 (a) Quantitation results achieved by the proposed algorithm for a representative fermentation broth in $90 \% \mathrm{H}_{2} \mathrm{O}$. The proposed algorithm took 12.4 seconds compared to $3.5 \mathrm{~min}$ utes taken by CRAFT [118], and does not require Regions of Interest (ROIs) to be specified. (b-c) Expansions of (a), displaying overlaid component spectra (from bottom to top, experimental, estimated, residual and component spectra). . 118

5.5 (a) Quantitation results obtained by the proposed algorithm for valeric acid (experimental, estimated and residual spectra). (b) Expansion of quantitation results displaying overlaid component spectra (from bottom to top, experimental, estimated, residual and component spectra). . . . . . . . . 119

6.1 The general scheme of a 2D-NMR experiment. . . . . . . . . 124

7.1 Schematic diagram of the 2D subband decomposition process with ideal frequency responses $\left\{H_{i, j}\left(f_{2}, f_{2}\right)\right\}_{i, j=1}^{2}$ [57]. . 143

$7.2{ }^{1} \mathrm{H}-{ }^{13} \mathrm{C}$ HSQC spectrum (Bruker DMX - 500Mhz) of nicotinamide adenine dinucleotide: (a)- stacked magnitude plot computed using FT, and (b)- reconstructed using (7.4). Processing time is 98.1 seconds. MSE is $5.8 \times 10^{-6} \ldots \ldots$. . . . 146

$7.3{ }^{1} \mathrm{H}-{ }^{1} \mathrm{H}$ COSY spectrum (Bruker DMX - 500Mhz) of HEPES: (a)- stacked magnitude plot computed using FT, and (b)reconstructed using (7.4). Processing time is 81.3 seconds. MSE is $1.2 \times 10^{-6} \ldots \ldots \ldots \ldots \ldots$. . . . . . . . . . . 148 
7.4 zTOCSY spectrum (Bruker DMX - 500Mhz) of gibberellic acid: (a)- computed using FT, and (b)- reconstructed using (7.4). Processing time is 73.7 seconds. MSE is $3.1 \times 10^{-7} \ldots 150$ 


\section{List of Tables}

3.1 Relationships between elements of the Fisher Information Matrix. . . . . . . . . . . . . . . . . . . 57

3.2 Calculation results for the interaction terms of the MRS, MRR and the amplitude-only model. . . . . . . . . . . . . . 81

3.3 Impact of water peak (MRS). . . . . . . . . . . . . . . . . 89

3.4 Impact of carbon satellites (MRS) . . . . . . . . . . . . . . 89

5.1 A comparison of experimental results. . . . . . . . . . . . 121

6.1 Comparison of acquisition time for multidimensional NMR experiments [47]. . . . . . . . . . . . . . . . . . . . . 129 


\section{List of Abbreviations}

\begin{tabular}{|c|c|}
\hline AWGN & Additive White Gaussian Noise \\
\hline ARMA & Auto Regressive Moving Average \\
\hline COSY & Correlation Spectroscopy \\
\hline CRLB & Cramer-Rao Lower Bound \\
\hline ECOSY & Exclusive Correlation Spectroscopy \\
\hline EID & Effective Independence Distribution \\
\hline EFI & Equivalent Fisher Information \\
\hline EPR & Electron Paramagnetic Resonance \\
\hline ESPRIT & $\begin{array}{l}\text { Estimation of Signal Parameters via Rotational Invariance } \\
\text { Technique }\end{array}$ \\
\hline FDM & Filter Diagonalisation Method \\
\hline FFT & Fast Fourier Transform \\
\hline FID & Free Induction Decay \\
\hline FIM & Fisher Information Matrix \\
\hline HMBC & Heteronuclear Multiple Bond Correlation \\
\hline HSQC & Heteronuclear Single Quantum Coherence Spectroscopy \\
\hline ICR & Ion Cyclotron Resonance \\
\hline
\end{tabular}


IPF Iterative Prefiltering

LF Leverrier-Faddeev method

LG Lobachevsky-Graeffe method

LP Linear Prediction

MIMO Multiple Input Multiple Output

MRI Magnetic Resonance Imaging

MRR Magnetic Resonance Relaxometry

MRS Magnetic Resonance Spectroscopy

MUSIC Multiple Signal Classification

NMR Nuclear Magnetic Resonance

NOESY Nuclear Overhauser Effect Spectroscopy

NQR Nuclear Quadruple Resonance

NUS Non-uniform Sampling

PCR Pulsed Corona Reactor

qNMR Quantitative NMR

ROESY Rotating-frame Overhauser Spectroscopy

SMA Steiglitz-McBride Algorithm

SNR Signal-to-Noise Ratio

SPA Stationary Phase Approximated

SVD Singular value Decomposition

TLS Total Least Squares

TOCSY Total Correlation Spectroscopy

UAV Uninhabited Aerial Vehicle 
${ }^{13} \mathrm{C}$ NMR Carbon-13

${ }^{1} \mathrm{H}$ NMR Hydrogen-1 


\section{Chapter 1}

\section{Introduction}

This thesis focuses on the problem of estimation of parameters of onedimensional (1D) and two-dimensional (2D) Nuclear Magnetic Resonance (NMR) signals. The work carried in this thesis will contribute to efficient processing of these signals. It will also be applicable to applications employing similar signal models, e.g., Electron Paramagnetic Resonance (EPR) [52], Ion Cyclotron Resonance (ICR) [45], and Microwave Resonance spectroscopy [33].

This chapter serves as an introduction to the thesis and puts forth the context of the research problems addressed therein. An outline of the chapter is as follows. The motivation behind the work carried out in this thesis is presented in Section 1, followed by a problem statement in Section 2. The organisation of the thesis and the original contributions are outlined in Sections 3 and 4, respectively. Section 5 highlights the publications originating from this work, succeeded by delineation of notations employed in the thesis in Section 6.

\subsection{Research motivation}

Data acquired from Nuclear Magnetic Resonance (NMR) experiments can be employed for rapid and efficient quantitation of compounds in chem- 


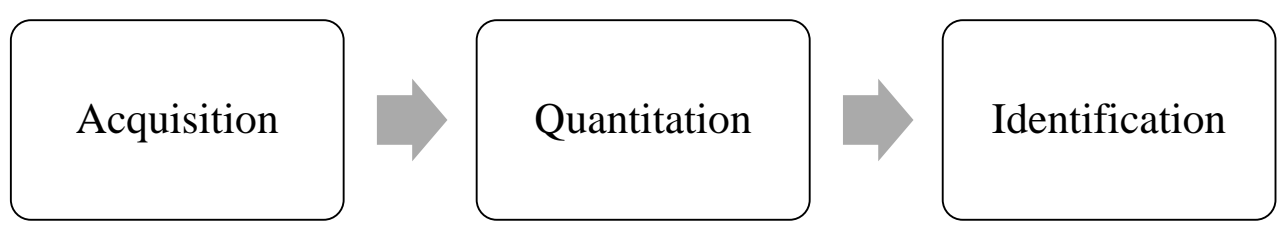

Figure 1.1: NMR data processing cycle [240].

ical samples. This makes NMR a powerful analytical tool for many research and industrial applications. For example, NMR has been shown to be gainfully employed in the food industry [19], in biological research [225], and in drug development [101].

NMR, under proper experimental conditions, causes the nuclei in a molecule to resonate. These resonances appear as peaks in the Fourier spectrum. The spectral parameters elicited from the peaks provide a fingerprint of the chemical components contained in the molecule. These fingerprints can be employed by the researchers to understand the chemical structure [88].

Signals originating from NMR experiments are processed in three stages [240], depicted in Figure 1.1:

1. Acquisition: the time-domain electromagnetic signal of a chemical sample is acquired from a NMR spectrometer,

2. Quantitation: the acquired signal is processed for feature extraction, and

3. Identification: based on the extracted features, compounds underlying the chemical sample are identified

Work carried in this thesis focuses on the second stage, i.e., quantitation. Signal acquired from NMR spectrometer is ideally modelled as a superposition of multiple damped complex exponentials (cisoids) in Additive White Gaussian Noise (AWGN) [216]. The number as well as the 
spectral parameters of the cisoids need to be estimated for the characterisation of the underlying chemicals. The estimator, or the algorithm, performing this task suffers from numerous difficulties in practice. These include [166]:

1. The number of cisoids in the signal is generally unknown,

2. Larger data lengths of recorded signal can be computationally prohibitive,

3. Dynamic range of signal can be large, i.e., very small and very large peaks coexist,

4. Cisoid density can be high, i.e., components overlap strongly,

5. Numerous distortions are also present, e.g., baseline of signal spectrum may be wavy (baseline distortion), the spectral shape of cisoids may deviate from the ideal cisoid shape (lineshape distortion), etc.

In view of these difficulties, an algorithm that can perform rapid and high-resolution quantitation of the NMR signals is a widely discussed research problem.

Various methods can be found in literature that endeavour to address these problems. They can be broadly subsumed under two categories [151,221]: model-based, which attempt to quantitate the NMR signal by minimising the sum of the squared errors between the recorded data and the signal model, and the so-called high resolution methods, in which quantitation is performed by separating the signal into signal and noise subspaces. Numerous other methods from different signal processing areas have also been employed. However, these methods suffer from performance issues - including limited resolution, high computational cost, and large estimate variance due to heavy apriori information reliance - when applied to high-complexity real-time NMR signals with the attributes mentioned in (1)-(5) above. 
Interpretable analytical results for the fundamental estimation theory tool, the Cramer Rao Lower Bound (CRLB), are unavailable for analytical evaluation of the performance of such estimation methods for the damped cisoid model with an arbitrary number of cisoids. Interpretable analytical CRLB results connote those that explicitly indicate the role of model parameters in determining the estimator performance. Jointly, these issues signify room for significant technical contributions to the area of NMR signal quantitation.

\subsection{Problem statement}

Motivated by this, the aim for this research can be summarised as follows: to explore a rigorous mathematical foundation for estimation of NMR signals and from the insights garnered therein, develop novel algorithms for their high-resolution, high-speed and apriori-information-independent processing. The mathematical foundation, based on the CRLB, should serve as the guiding hand in developing the algorithms that should be able to:

- Estimate, accurately and efficiently, the number and parameters of cisoids embedded in real-time NMR signals with:

- large data length,

- large number of cisoids,

- large dynamic range, and

- a large number of dense overlapping peaks

in the presence of:

- noise,

- distortions, i.e., baseline, lineshape, etc., and

- possibly any other uncertainty arising in the NMR signal from potentially undiscovered sources 
and in the absence of:

- aprior signal/chemical knowledge in any form.

These goals are accomplished by deriving novel analytical CRLB results for parameter estimation of 1D NMR signals, and developing novel algorithms for processing of data acquired from 1D and 2D NMR (mainly proton) experiments. These outcomes should serve to advance the mathematical knowledge in the theory of estimation of 1D NMR signals, and help to overcome the limitations of current methods available for the quantitation of 1D and 2D NMR signals, providing, at the same time, significant advances in their performance as well. Above outcomes should also bring innovative parameter estimation insights and novel quantitation applications to numerous related areas in spectroscopy and signal processing.

\subsection{Organisation of the thesis}

Remainder of the thesis is organised as follows:

Chapter 2 (Introduction to NMR) provides an introduction to the background knowledge of NMR. Historical development, NMR physics, the basics of 1D and 2D-NMR experiments, and the chemical concepts relevant to the thesis are also discussed.

Chapter 3 (Parameter Estimation in NMR: Some general results on the Cramer Rao Lower Bound) presents the results achieved for the first part of our work, i.e., the analytical evaluation of the CRLB for parametric estimation in NMR, in the following order. Firstly, background to the problem, models of importance in this regard and, for the models considered, the significance of the results reported are discussed. Secondly, a formal mathematical statement of the problem and the methods employed to solve it are elaborated. Finally, 
the derived results and their application to real-time NMR data, in conjunction with the key insights garnered in this regard, are presented.

Chapter 4 (Quantitation Methods for 1D-NMR Signals) presents a review of 1D-NMR signal quantitation methods available in the literature. They are grouped in three categories: time-domain, frequency-domain and combined time-frequency domain methods. Representative approaches in each category are discussed along with the advantages and limitations of each.

Chapter 5 (Quantitation Algorithm for 1D-NMR Signals) reports the development of a high-speed, high-resolution and aprioriinformation-free quantitation algorithm for 1D-NMR signals. The chapter comprises a formal introduction to the problem, the proposed solution, the developed algorithm and a performance comparison with state-of-the-art methods.

Chapter 6 (Quantitation Methods for 2D-NMR Signals) presents background to the 2D-NMR, a brief discussion of well-established 2D-NMR experiments, the limitations encountered in experimental data acquisition/processing, and a review of quantitation algorithms developed to process the 2D-NMR data strictly on a uniform sampling grid.

Chapter 7 (Quantitation Algorithm for 2D-NMR Signals) reports, in a manner similar to Chapter 5 , the development of an algorithm for rapid, high-resolution and apriori-information-free quantitation of 2D-NMR data.

Chapter 8 (Conclusions and Future Work) concludes the thesis by reviewing the contributions and discussing the potential research directions. 


\subsection{Original contributions}

The original contributions of this thesis include:

1. Analytical evaluation of the CRLB: Chapter 3 reports analytical expressions for the CRLB on the variance of unbiased parameter estimates of damping factors, frequencies and complex amplitudes of an arbitrary number of damped cisoids embedded in AWGN (model representing the FID signal in 1D-NMR data). The expressions display the explicit relationship of the CRLB for each distinct parameter with respect to all the free model parameters, i.e., the number of cisoids, their amplitudes, phases, frequencies, damping factors, Signal-to-Noise Ratio (SNR) and the sampling rate, without imposing restriction on any of them. The reported results do not involve auxiliary expressions for their explanation and are readily interpretable in terms of all the free model parameters. In addition to the CRLB, analytical expressions for the determinant and the condition number of the Fisher Information Matrix (FIM) associated with the damped cisoid model are also reported.

Two special cases of the damped cisoid model, the Magnetic Resonance Relaxometry (MRR) model and the amplitude-only model (employed in quantitative NMR - qNMR), are also considered, and analytical expressions for the CRLB on the unbaised parameter estimates of these models, as well as for the determinant and condition number of their respective FIMs, are reported. Some auxiliary results for the above-mentioned models are also presented, i.e., on the multiplicity of the eigenvalues, their separation, and the factorisation of the characteristic polynomial associated with their respective FIMs. These results, as such, have not been previously reported in literature.

The reported theoretical results, when applied to experimental NMR data, successfully accounted for various physical and chemical phe- 
nomena observed in 1D-NMR data and quantified their impact on the accuracy of an unbiased estimator as a function of both model and experimental parameters, i.e., influence of prior knowledge, peak multiplicity, multiplet symmetry, solvent peak, carbon satellites, baseline distortion, signal truncation, and the second-order effects observed in spin-coupled NMR systems.

2. High-resolution, high-speed, apriori-information-free quantitation algorithm for 1D NMR data: Chapter 5 reports the development of a parametric algorithm for high-resolution, high-speed and apriori-information-free quantitation of 1D-NMR data. The developed algorithm is able to convert the time-domain FID data into a table of estimated amplitudes, phases, frequencies and damping factors - without requiring any previous knowledge or preprocessing (i.e., base and phase correction, apodization, noise removal, etc.). Application of the developed algorithm on experimental proton FID data attested to its superior performance, in terms of speed, resolution and automation, when compared to the state-of-the-art in this area.

3. High-resolution, high-speed, apriori-information-free quantitation algorithm for 2D NMR data: Chapter 7 reports the development of a 2D parametric algorithm for high-resolution, high-speed and apriori-information-free quantitation of 2D-NMR data. The developed algorithm is able to convert the time-domain $2 \mathrm{D}-\mathrm{NMR}$ data into a table of estimated amplitudes, phases, 2D-frequencies and damping factors. The algorithm achieves this in a highly accurate, time-efficient and automated fashion, without requiring any previous knowledge or preprocessing (i.e., linear prediction, zero-filling, apodization, etc.). The ability of the algorithm to convert raw 2DNMR data into a table of estimates makes it directly applicable to the time-domain data obtained from a variety of homonuclear, het- 
eronuclear and spatial 2D-NMR experiments. When applied to the data acquired from various 2D-NMR experiments in this regard, the proposed algorithm yielded excellent quantitation results in terms of speed, resolution and automation - markedly surpassing, in these respects, the results achieved by the previously published techniques for the analysis of 2D-NMR data.

\subsection{List of publications}

1. M.A.R. Anjum, P.A. Dmochowski, and P.D. Teal. A subband Steiglitz-McBride algorithm for automatic analysis of FID data. Magnetic Resonance in Chemistry, February 2018.

2. M.A.R. Anjum, P.D. Teal, and P.A. Dmochowksi. An adaptive subband decomposition approach for high-resolution NMR data analysis. In 14th International Conference on Magnetic Resonance Microscopy (ICMRM), Halifax, Canada, August 2017.

3. M.A.R. Anjum, P.D. Teal, and P.A. Dmochowksi. Some signal processing issues in NMR. IEEE Postgraduate Presentation Event, Wellington, NEW ZEALAND, August 2016. BEST PAPER AWARD.

4. M.A.R. Anjum, P.A. Dmochowksi, and P.D. Teal. Parameter estimation in nuclear magnetic resonance: some general results on the Cramer-Rao lower bound. Journal of Mathematical Chemistry. In review.

5. M.A.R. Anjum, P.A. Dmochowksi, and P.D. Teal. 2D subband Steiglitz-McBride algorithm for automatic analysis of 2D-NMR data. Magnetic Resonance in Chemistry. In review.

6. M.A.R. Anjum, P.A. Dmochowksi, and P.D. Teal. Analytical CrameRao Lower Bound Expressions for Parameter Estimation of Damped 
Complex Exponential Signals in Noise. IEEE International Conference on Acoustics, Speech Signal Processing (ICASSP), Brighton, UK, May 2019. In review.

7. M.A.R. Anjum, P.A. Dmochowksi, and P.D. Teal. Analytical CramerRao lower bound expressions for parameter estimation of multiexponential signals in noise. To be submitted.

\subsection{Notations}

The rest of the thesis employs lower-case italic characters, such as $x$, to represent scalars; lower case bold characters, such as $\boldsymbol{x}$, for vectors; upper case bold characters, such as $\mathbf{X}$, for matrices; the superscript asterisk * to denote the conjugate of a complex number; $\{\bullet\}^{T}$ for the transposition and $\{\bullet\}^{H}$ for conjugate transposition of a matrix, and equally a vector; $\{\hat{\bullet}\}$ for the estimated value of a parameter; and $\Re\{\bullet\}$ and $\Im\{\bullet\}$ to denote the real and imaginary parts respectively. 


\section{Chapter 2}

\section{Introduction to NMR}

\subsection{Background and significance}

Nuclear magnetic resonance (NMR) is a well established technique with a wide range of applications in physics, chemistry, biology, geology and medicine [105]. Historically, it took NMR almost sixty years to achieve its currently popular status. NMR technology began with the independent discoveries of two scientific groups, one lead by Bloch and the other by Purcell [16]. For their contribution, both were jointly awarded the 1952 Nobel Prize in Physics.

NMR found direct application in chemistry with the discovery of chemical shift when it was observed that two nitrogen atoms in ammonium nitrate induced two different signals [167]. This observation was subsequently confirmed by the detection of three lines in the spectrum of ethanol. This, in turn, gave rise to significant commercial activity in NMR, the first commercial NMR spectrometer being produced in 1952. Further discoveries ensued in 1953, when it was observed that the saturation of electrons in metals caused a nuclear polarisation increase. This effect was named after its discoverer as the Overhauser effect [160]. This was the first evidence that spins can actually communicate; the method was later used to study other interactions between nuclei, e.g., spin-spin 
coupling in 1961 [76].

In the early ears of its development, the sensitivity of NMR was a significant issue. This promoted activity on the development of stronger electromagnets. These were not fully realised until the early 60 s when superconducting magnets emerged in the market. This lead to the generation of much higher 100MHz spectra [69]. However, technical challenges of magnetic stability and inhomogeneity remained to be tackled. Progress in this direction was made when the first $200 \mathrm{MHz}$ spectrum was generated in 1964 [157]. Further increase in sensitivity was achieved with the introduction of the Fourier transformed NMR (FT-NMR) in 1966 [68]. This allowed simultaneous excitation, and later, separation of various chemical signals. The discovery of FT-NMR opened the doors for various pulse sequence methods.

Towards the end of the 50s, medicinal applications of NMR spectroscopy were also being considered [156]. The NMR spectra of red blood cells in humans, known as erythrocytes, were studied to elicit their properties, along with human saliva [161]. A few years later, NMR was also applied to ascertain abnormal properties of tumors [165]. At the same time, biological molecules were also being studied for their structural properties using NMR [108]. The majority of the research in this direction was focused on peptides and paramagnetic proteins. The greatest challenge was posed by the peak of the water signal whose amplitude was many times larger than that of the signal of interest. This problem was overcome by another breakthrough when, in 1976, Ernst et. al introduced an additional axis in NMR spectra for the assignment of overlapping frequencies. This effectively marked the birth of high-resolution two-dimensional NMR (2D-NMR) [11].

2D-NMR was rapidly identified as a prospective structural tool by researchers working in the field of biomolecules [145]. The method was seen to be feasible in this area despite the limited computational resources available at that time. Its practical use however was hindered for many 
years by the various technical difficulties that arose due to the high concentration of proteins. It was not until 1985 when the first 2D-NMR structure of protein was published [233]. Further improvement in resolution lead to the introduction of 3D-NMR in 1990 which was subsequently used to probe the structures of biological macromolecules [184].

Today, NMR is ubiquitous. According to the areas in which it finds its applications, it can be broadly represented by three overlapping regions: spectroscopy, relaxometry and imaging as indicated in Figure 2.1 [77]. Spectroscopy allows characterisation based on chemical properties; relaxometry allows characterisation based on physical properties; and imaging allows characterisation based on spatial properties.

NMR spectroscopy has surpassed the X-ray crystallography for structural determination in biology and chemistry [16]. It has replaced Infrared (IR) spectroscopy as a principal investigation tool for forensic analysis [230]. It is also being considered as an alternative to Positron Emission Tomography (PET) spectroscopy for metabolic imaging in medicine [164]. Furthermore, it is finding its way into ever new applications as a substitute to the orthodox techniques, e.g., Near Infrared (NIR) in determination of food quality and authenticity [174]. All this can be attributed to the numerous advantages NMR has to offer over the competing techniques.

\subsubsection{Advantages of NMR}

The principal advantages of NMR are briefly summarised as follows:

1. Non-invasiveness,

2. Non-directional preference,

3. Multi-parametric measurement ability,

4. Immunity to optical opaqueness,

5. Inherently quantitative (signal received directly from the nuclei), 


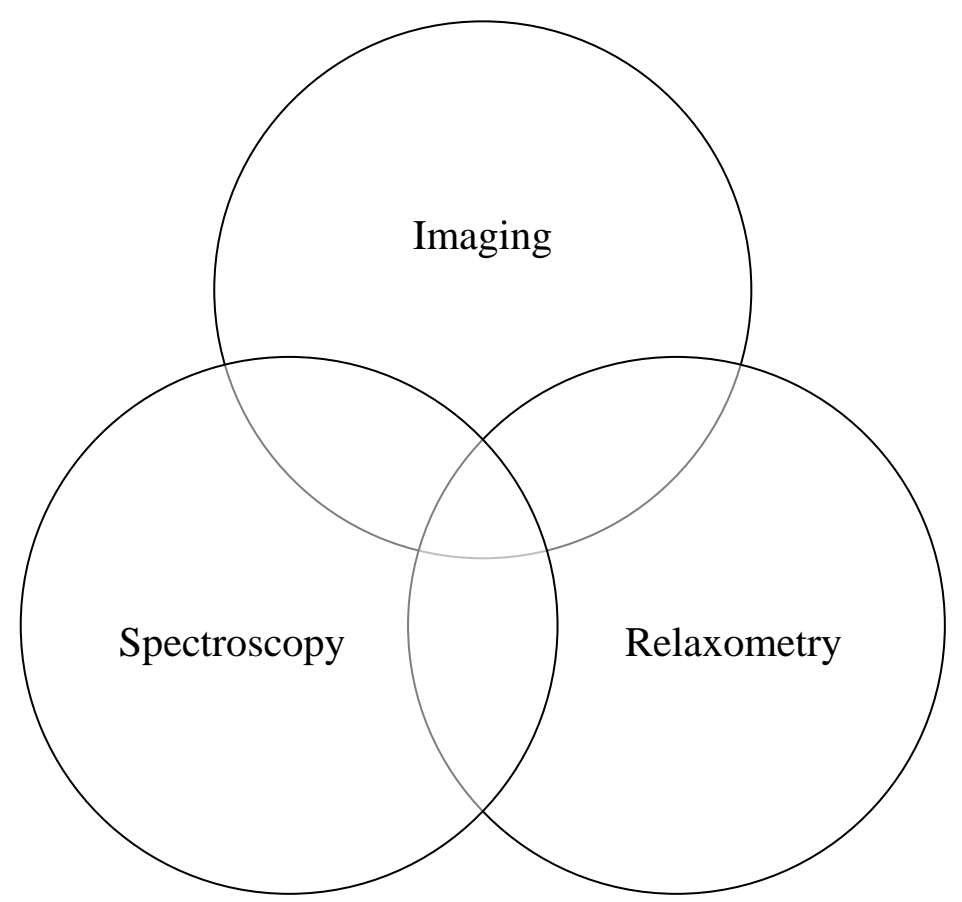

Figure 2.1: A schematic representation of NMR as partially overlapping areas representing the three main applications [77].

6. A direct method, and

7. Multi-nuclear method

\subsection{NMR physics}

In the following sub-sections, the basic theory behind NMR is discussed.

\subsubsection{Spin and the spin angular momentum}

NMR is based on the quantum mechanical phenomenon known as spin angular momentum. The atomic beam experiment, conducted by Stern and Gerlach in 1922, demonstrated the existence of spatial quantization 
[29]. They observed the splitting of a collated atomic beam of silver in the presence of an external magnetic field. This implied the existence of a non-classical spin. The theoretical explanation for the spin was provided by Dirac in 1928 [95]. Spin is now known as an intrinsic property of the atomic nuclei and the source of their spin angular momentum, denoted by $\boldsymbol{I}$. Spin angular momentum is quantized, i.e., it can only take $2 I+1$ discrete values in a particular observed direction, e.g., $I_{z}=\bar{h} m_{z}=\bar{h}\{-I,-I+1, \ldots, I-1, I\} . I_{z}$ is the spin-component, $\bar{h}$ is the reduced Planck's constant (i.e., $h / 2 \pi$ ), and $m_{z}$ is the spin projection quantum number along $z$-axis. $I$ is the principal spin quantum number. Nuclei in which protons are evenly paired have zero spin angular momentum. Others have a non-zero spin angular momentum, e.g., hydrogen has a half-integer spin $I=1 / 2$ due to a single proton. This non-zero spin angular momentum $\boldsymbol{I}$ generates a dipole moment $\boldsymbol{\mu}$ in the associated nuclei given by:

$$
\boldsymbol{\mu}=\gamma \boldsymbol{I}
$$

where $\gamma$ is the constant of proportionality, known as the gyromagnetic ratio, e.g., the $\gamma$ for proton $\left({ }^{1} \mathrm{H}\right)$, Flourine $\left({ }^{19} \mathrm{~F}\right)$ and Phosphorus $\left({ }^{31} \mathrm{P}\right)$ are $267.513,251.662$ and $108.291 \times 10^{6} \mathrm{rad} \mathrm{s}^{-1} \mathrm{~T}^{-1}$ receptively [91]. Half-integer spin nuclei have two eigenstates which, in the presence of a static external magnetic field $\boldsymbol{B}_{0}$, become non-degenerate. The difference in the energy levels $E$ is determined by:

$$
E=\boldsymbol{\mu} \cdot \boldsymbol{B}_{0}=-\mu_{x} B_{x 0}-\mu_{y} B_{y 0}-\mu_{z} B_{z 0}
$$

$\boldsymbol{B}_{\mathbf{0}}$ is normally taken along the $z$-axis as indicated in Figure 2.2. This reduces the above expression to:

$$
E=-\mu_{z} B_{0}=-\gamma \bar{h} m_{z} B_{0}
$$

Consequently, nuclear spins will possess different energies in the presence of a non-zero magnetic field. This process is known as Zeeman splitting 


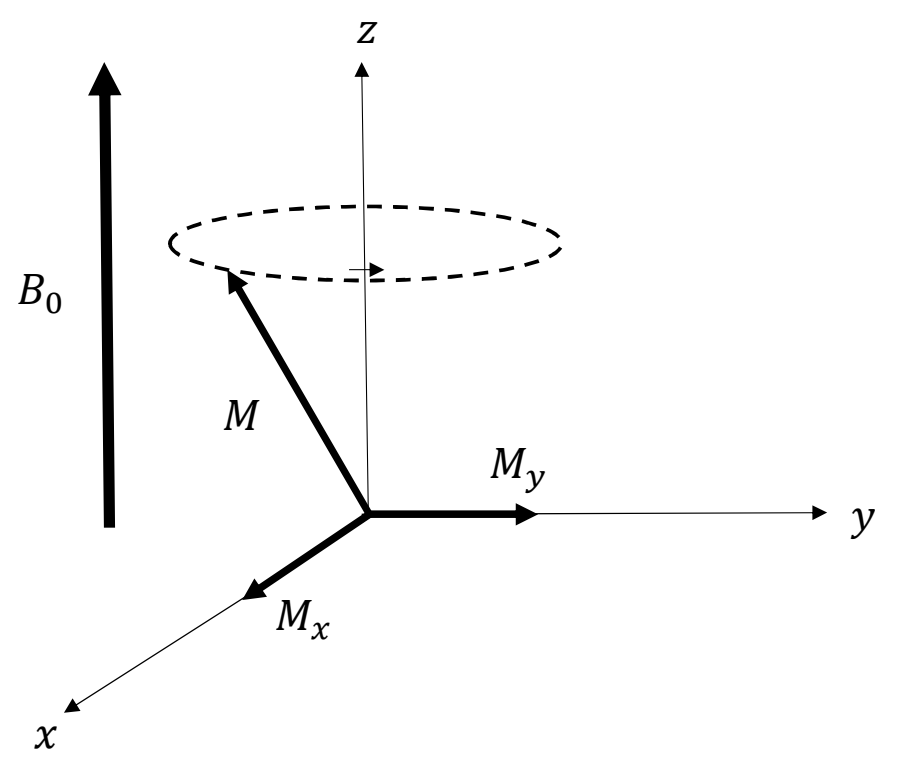

Figure 2.2: Physical basis of NMR [36].

[36]. The net nuclear magnetic moment that results due to the application of $B_{0}$ is called magnetisation $M$. It is defined by:

$$
M=\frac{N \gamma^{2} h^{2} I(I+1)}{3 k T} B_{0}=\chi_{0} B_{0}
$$

where $\chi_{0}$ represents the magnetic susceptibility of the spin system, $N$ the number of nuclei, $k$ Boltzmann's constant, and $T$ the absolute temperature. This relationship is also known as Curie's Law [35]. Due to the interaction with $B_{0}$, the dipoles precess around the $z$-axis. The angular frequency of this precession $\omega_{0}$ is given by:

$$
\omega_{0}=\gamma B_{0}
$$

where $\omega_{0}$ (or $v=\omega_{0} / 2 \pi$ ) is known as the Larmor frequency [36]. 


\subsubsection{Spin relaxation}

In the equilibrium state, the $M$ vector is in the longitudinal direction, i.e., along the $z$-axis as indicated in Figure 2.2. This results from an uneven population in quantum levels and random phases of the individual spins. If external energy is applied, typically an oscillating electromagnetic field $B_{1}, M$ will move from its equilibrium position to align with $B_{1}$. The recovery of the equilibrium state, i.e., the relaxation process, involves both restoration of population levels and randomisation of the individual spins. The relaxation occurs due to the interaction of oscillating magnetic fields within the molecules and is caused by molecular collisions. The relaxation process is unique to the molecular environment. Consequently, a unique fingerprint of frequencies will be produced for each chemical group which can be used for their identification. One point worth mentioning here is that the collision process in NMR leads to a lifetime in milliseconds for the excited molecular states which is longer than that of other spectroscopic techniques. This, according to Heisenbergs uncertainty principle, will lead to [36]:

- The NMR signal being comparatively narrower in bandwidth, and

- Being more manipulatable in the time time-domain after excitation.

These two benefits are crucial to the success of NMR.

\subsubsection{Spin-Lattice relaxation time $\left(T_{1}\right)$}

When a radio wave $B_{1}$, with a Larmor frequency $v$, is applied to the sample, its causes the transition of populations from the lower energy level to the higher one. Restoration of the equilibrium population levels occurs by electromagnetic radiation. Magnetisation $M$ also recovers its equilibrium position, i.e., along $B_{0}$. This recovery process is exponential and is described by a spin-lattice relaxation time $T_{1} . T_{1}$ is also known as the enthalpic 
component [36] of the equilibrium restoration, as it involves the restoration of equilibrium populations.

\subsubsection{Spin-Spin relaxation time $\left(T_{2}\right)$}

Contrary to the restoration of population levels, the spin-spin relaxation time is connected with the randomisation of individual spins. Represented by $T_{2}$, it is also exponential in decay and governs the return of the individual spins to random phases. $T_{2}$ is also known as the anisotropic component [36] of the equilibrium restoration, as it involves the randomisation of spin phases.

\subsubsection{The rotating frame of reference}

Under the influence of $B_{0}$, there is a net magnetisation $M$ along the direction of $B_{0}$, generally taken along the $z$-axis as indicated in Figure 2.2. When $B_{1}$ is applied along the $x$-axis, in the form of an RF pulse with a frequency $\omega_{1}$, then under the influence of these two magnetic fields, $M$ will begin to precess simultaneously around $B_{0}$, with a frequency $\omega_{0}=\gamma B_{0}$, and $B_{1}$, with a frequency $\omega_{1}=\gamma B_{1}$. Spins around $B_{0}$ can be considered from a laboratory frame of reference, i.e., the observation system from where $B_{0}$ has been applied. If this frame of reference is exchanged for the one that rotates at $\omega_{0}$, spins which have a frequency equal to $\omega_{0}$ will experience the effect of $B_{1}$ only and precess about its axis. Other spins will precess around a magnetic field that is effectively the vector sum of $B_{0}$ and $B_{1}$. The net effect of $B_{1}$ on $M$, since $B_{1}$ is applied for a short time, is the partial revolution of $M$ around the $B_{1}$ axis, known as tilt [36].

\subsubsection{Bloch equations of magnetisation motion}

With all the background in place, the evolution of spins in an external homogeneous magnetic field shall now be discussed. The equations which 
describe this process are known as Bloch equations after Felix Bloch who formulated them. The formulation commences by observing that the bulk magnetisation $\mathbf{M}$ of the sample is equal to the vector sum of individual dipole moments $\left(\mu_{i}\right)$ [129]:

$$
\mathbf{M}=\sum_{i} \mu_{i}
$$

In terms of the net angular momentum $\mathbf{L}, \mathbf{M}$ is given by:

$$
\mathbf{M}=\gamma \mathbf{L}
$$

In the presence of an external magnetic field $\mathbf{B}, \mathbf{M}$ experiences a torque and the net angular momentum of the system is changed by:

$$
\frac{d \mathbf{L}}{d t}=\mathbf{M} \times \mathbf{B}
$$

which can be re-written as:

$$
\frac{d \mathbf{M}}{d t}=\gamma \mathbf{M} \times \mathbf{B}
$$

The cross-product can be expanded as:

$\left|\begin{array}{ccc}\mathbf{i} & \mathbf{j} & \mathbf{k} \\ M_{x} & M_{y} & M_{z} \\ B_{x} & B_{y} & B_{z}\end{array}\right|=\left(M_{y} B_{z}-M_{z} B_{y}\right) \mathbf{i}+\left(M_{z} B_{x}-M_{x} B_{z}\right) \mathbf{j}+\left(M_{x} B_{y}-M_{y} B_{x}\right) \mathbf{k}$

which can be expanded in terms of the individual components as:

$$
\begin{aligned}
& \frac{d M_{x}}{d t}=\gamma\left(M_{y} B_{z}-M_{z} B_{y}\right) \\
& \frac{d M_{y}}{d t}=\gamma\left(M_{z} B_{x}-M_{x} B_{z}\right) \\
& \frac{d M_{z}}{d t}=\gamma\left(M_{x} B_{y}-M_{y} B_{x}\right)
\end{aligned}
$$


Since the magnetic field is applied along the $z$-axis (i.e., $B_{x}=B_{y}=0$ ), dipoles precess around $B_{z}$.

$$
\begin{aligned}
& \frac{d M_{x}(t)}{d t}=\gamma M_{y}(t) B_{z} \\
& \frac{d M_{y}(t)}{d t}=-\gamma M_{x}(t) B_{z} \\
& \frac{d M_{z}(t)}{d t}=0
\end{aligned}
$$

In the absence of relaxation, the solution of these equations is:

$$
\begin{aligned}
& M_{x}(t)=M_{x} \cos (\omega t)+M_{y} \sin (\omega t) \\
& M_{y}(t)=M_{y} \cos (\omega t)-M_{x} \sin (\omega t)
\end{aligned}
$$

Spin-lattice and spin-spin relaxation are first order processes with relaxation times $T_{1}$ and $T_{2}$ respectively. Including the relaxation terms and given that $B_{z}=B_{0}$ and $B_{x}=B_{y}=0$ :

$$
\begin{aligned}
& \frac{d M_{x}(t)}{d t}=\omega_{0} M_{y}(t)-\frac{M_{x}(t)}{T_{2}} \\
& \frac{d M_{y}(t)}{d t}=-\omega_{0} M_{x}(t)-\frac{M_{y}(t)}{T_{2}} \\
& \frac{d M_{z}(t)}{d t}=-\frac{\left(M_{z}(t)-M_{0}\right)}{T_{1}}
\end{aligned}
$$

with $\omega_{0}=\gamma B_{0}$. These equations represent Bloch's three approximations which he used to derive them. His first assumption was that the magnetisation will return to the $z$-axis over time in the act of forgetting its state. This is described by the equation containing the $T_{1}$ term. Secondly, upon the interaction with $B_{1}$, the magnetisation will acquire a transversal component. Thirdly, under magnetic inhomogeneities, the spins, though assumed free, will experience a change in their frequencies, and undergo a decoherence process. The last two approximations are described by the set 
of equations containing in the $T_{2}$ term. Solving (2.14) yields:

$$
\begin{aligned}
& M_{z}(t)=M_{z}(0) e^{-t / T_{1}}+M_{0}\left(1-e^{-t / T_{1}}\right) \\
& M_{x}(t)=\left\{M_{x}(0) \cos \left(\omega_{0} t\right)-M_{y}(0) \sin \left(\omega_{0} t\right)\right\} e^{-t / T_{2}} \\
& M_{y}(t)=\left\{-M_{x}(0) \sin \left(\omega_{0} t\right)+M_{y}(0) \cos \left(\omega_{0} t\right)\right\} e^{-t / T_{2}}
\end{aligned}
$$

When an RF pulse with frequency $\omega$ is applied in the transverse plane:

$$
\begin{aligned}
B_{x}(t) & =B_{1} \cos (\omega t) \\
B_{y}(t) & =B_{1} \sin (\omega t) \\
B_{z} & =B_{0}
\end{aligned}
$$

The Bloch equations become:

$$
\begin{aligned}
& \frac{d M_{x}(t)}{d t}=\omega_{0} M_{y}(t)-\omega_{1} M_{z}(t) \sin (\omega t)-\frac{M_{x}(t)}{T_{2}} \\
& \frac{d M_{y}(t)}{d t}=-\omega_{0} M_{x}(t)+\omega_{1} M_{z}(t) \cos (\omega t)-\frac{M_{y}(t)}{T_{2}} \\
& \frac{d M_{z}(t)}{d t}=-\omega_{1}\left[M_{x}(t) \sin (\omega t)+M_{y}(t) \cos (\omega t)\right]-\frac{\left(M_{z}(t)-M_{0}\right)}{T_{1}}
\end{aligned}
$$

In a rotating frame of reference, following transformation [173]:

$$
\begin{aligned}
& M_{x}^{\prime}(t)=M_{x}(t) \cos (\omega t)-M_{y}(t) \sin (\omega t) \\
& M_{y}^{\prime}(t)=M_{x}(t) \sin (\omega t)+M_{y}(t) \cos (\omega t)
\end{aligned}
$$

yields:

$$
\begin{aligned}
& \frac{d M_{x}^{\prime}(t)}{d t}=-\left(\omega-\omega_{0}\right) M_{y}^{\prime}(t)-\frac{M_{x}^{\prime}(t)}{T_{2}} \\
& \frac{d M_{y}^{\prime}(t)}{d t}=\left(\omega-\omega_{0}\right) M_{x}^{\prime}(t)-\frac{M_{y}^{\prime}(t)}{T_{2}}+\omega_{1} M_{z}(t) \\
& \frac{d M_{z}(t)}{d t}=-\omega_{1} M_{y}^{\prime}(t)-\frac{\left(M_{z}(t)-M_{0}\right)}{T_{1}}
\end{aligned}
$$

These equations characterise the motion of magnetisation under a given set of initial conditions. 


\subsection{The 1D-NMR experiment}

The basic experiment in NMR is the one-dimensional or (1D) NMR experiment [38]. It is performed by the application of a $90^{\circ}$ pulse along the $x$-axis with following initial conditions:

$$
\begin{aligned}
& M_{x}=0 \\
& M_{y}=M_{0} \\
& M_{z}=0
\end{aligned}
$$

In this case, the evolution of the resulting magnetisation processes according to (2.19) is given by:

$$
\begin{aligned}
& \frac{d M_{x}^{\prime}(t)}{d t}=-\left(\omega-\omega_{0}\right) M_{y}^{\prime}(t)-\frac{M_{x}^{\prime}(t)}{T_{2}} \\
& \frac{d M_{y}^{\prime}(t)}{d t}=\left(\omega-\omega_{0}\right) M_{x}^{\prime}(t)-\frac{M_{y}^{\prime}(t)}{T_{2}}
\end{aligned}
$$

yielding the solution:

$$
\begin{aligned}
& M_{x}^{\prime}(t)=\left\{M_{x}^{\prime}(0) \cos (\Delta \omega t)+M_{y}^{\prime}(0) \sin (\Delta \omega t)\right\} e^{-t / T_{2}} \\
& M_{y}^{\prime}(t)=\left\{M_{x}^{\prime}(0) \sin (\Delta \omega t)+M_{y}^{\prime}(0) \cos (\Delta \omega t)\right\} e^{-t / T_{2}}
\end{aligned}
$$

with $\Delta \omega=\omega-\omega_{0}$. The initial conditions in (2.20) lead to $M_{x}^{\prime}(0)=0$ and $M_{y}^{\prime}(0)=M_{0}$ in (2.18). As a result, (2.22) becomes:

$$
\begin{aligned}
& M_{x}^{\prime}(t)=M_{0} \sin (\Delta \omega t) e^{-t / T_{2}} \\
& M_{y}^{\prime}(t)=M_{0} \cos (\Delta \omega t) e^{-t / T_{2}}
\end{aligned}
$$

Treating the $x$ and $y$ as real and imaginary components of a complex variable, $M_{x}^{\prime}(t)$ and $M_{y}^{\prime}(t)$ can be jointly written as:

$$
M_{y}^{\prime}(t)+i M_{x}^{\prime}(t)=M_{0} e^{i \Delta \omega t} e^{-t / T_{2}}
$$

or

$$
M_{x y}^{\prime}(t)=M_{0} e^{\left(i \Delta \omega-1 / T_{2}\right) t}
$$

The signal $M_{x y}^{\prime}(t)$ is termed as the Free Induction Decay (FID) signal. The envelope of the FID is signal is described by the transverse relaxation time $T_{2}$. An example FID signal is shown in Figure 2.3. 


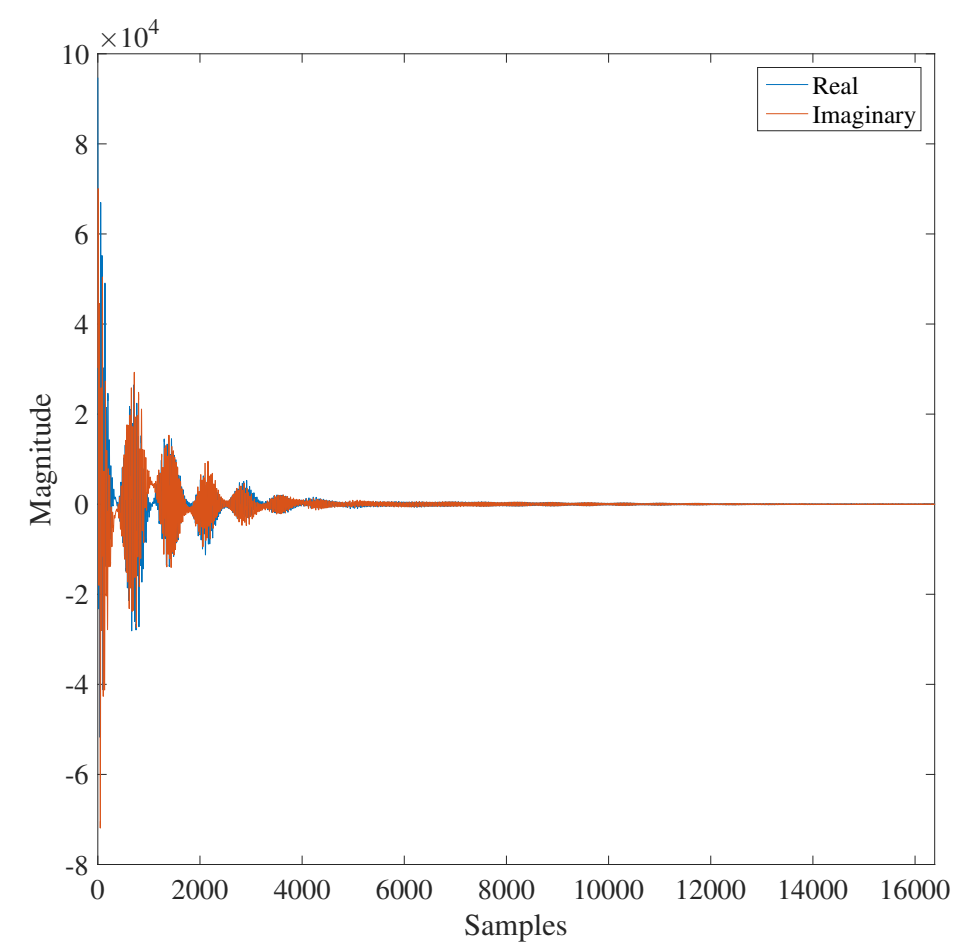

Figure 2.3: Real and imaginary parts of FID of ethanol recorded on Spinsolve Benchtop NMR (Magritek; Wellington, New Zealand).

\subsubsection{The FID signal model}

The FID signal in (2.25) comprises of a real part and an imaginary part corresponding to the orthogonal components of the projection of $M$ in the transversal plane respectively. As a result, application of the excitation pulse in 1D-NMR experiment is followed by the detection of potential induced in receiver coils placed in transversal plane, a technique known as quadrature detection. If the chemical sample consists of more than one component, the resulting FID signal equals the sum of the FIDs of individual 
components [216]. The FID is acquired in discrete-time.

$$
f(m \delta)=\sum_{n=1}^{N} \beta_{n} e^{\left(i \Delta \omega_{n}-1 / T_{2}\right) m \delta}+v(m) \quad m \in[0, M-1]
$$

where $M$ represents the number of data points acquired, $\delta$ is the acquisition delay between two adjacent samples, $n$ signifies the number of individual spins, and $\beta_{n}$ characterises the complex amplitude of each complex decaying exponential. $v(m)$ represents the Gaussian noise which cumulatively represents the effects of random molecular motion in the sample, thermal noise in the receiver coils, and imperfections in the processing equipment [38]. The FID data is usually collected until the signal has decayed completely [36].

\subsubsection{Practical features}

Due to inherent magnetic inhomogeneities, the FID signal decays faster than the rate dictated by $T_{2}$ [38]. This requires the FID signal to be sampled at a high rate in order to resolve the embedded complex exponentials - a consequence of the Nyquist sampling theorem. This in turn generates a large number of FID data samples for processing, generally $\geq 2^{14}$ for a typical 1D-NMR experiment. Also, the number of individual complex exponentials in an FID signal is generally unknown in advance. Further, the variations in the chemical environment, along with the magnetic inhomogeneities, increase the complexity of the generated FID signal by introducing numerous artefacts, such as distortion in the anticipated Lorentzian peak shapes and the base-line of the FID spectrum [241]. Moreover, weak and overlapping peaks may be observed in the FID spectrum, the former due to chemical impurities and/or carbon satellites [195] and the latter due to the occurrence of the same type of nuclei in different chemical environments [8]. These peaks overlap and merge in the resultant FID spectrum, making it even more complex. 


\subsubsection{Motivation for work carried in Chapter 5: Quantita- tion algorithm for 1D-NMR signals}

The general objective of industrial spectroscopy is to estimate the number of chemically different components in a sample, as well as their characteristics and concentrations. This is equivalent to the estimation of the number of complex damped exponentials (cisoids) constituting an FID signal as well as their parameters, i.e., complex amplitude, frequency and damping factor. However, the above mentioned practical features of an FID signal make the accurate quantitation of an FID signal much more complicated in practice. FID quantitation methods that endeavour to tackle this problem suffer from one or more of the following issues: speed, accuracy, resolution and requiring operator input. Consequently, there is a need for a method that strikes a balance among all of the four properties. A detailed survey outlining the state of the art in this area will be presented in Chapter 4.

\subsubsection{Motivation for work carried in Chapter 3: The CRLB}

The above-mentioned issues concerning the performance of an FID quantitation method are not exhaustive. The universal performance benchmark that characterizes such quantitation methods, i.e., the Cramer-Rao Lower Bound (CRLB), does not have closed form expressions, exact or approximate, that display the explicit dependence of the CRLB on the free model parameters for the number of cisoids greater than three [37]. This imposes a limit on the degrees of freedom in which the performance of an estimator can be evaluated in terms of its free parameters. Consequently, this avenue needs further exploration. The state-of-the-art in this area will be explored in Chapter 3. 


\subsection{The 2D-NMR experiment}

The NMR research that took place in the 1970s was focused on devising new methods for extracting greater information from the chemical samples, especially for the assignment of overlapping frequencies. The basic idea was to generalise the pulse sequences into three components: excitation, evolution and acquisition. The "evolution" component, which is lacking in 1D-NMR experiments, forms the key to $2 \mathrm{D}$ experiments. In the $2 \mathrm{D}$ case, once the excitation pulse is applied, the sample is allowed to evolve for a certain period of time, the evolution time, before the acquisition process begins. The acquired FID signal varies with changes in the evolution time. This adds another dimension to the signal which in turn enables the study of the evolution of coupling information between the resonances of a molecule. Consequently, 2D-NMR is employed to study macro-molecular structures. A wide variety of 2D-NMR techniques are available for this purpose, e.g., Diffusion Ordered Spectroscopy (DOSY) [190], Nuclear Overhauser Effect Spectroscopy (NOESY) [121], Correlation Spectroscopy (COSY) [73], etc.

\subsubsection{Motivation for work carried in Chapter 7: Quantita- tion algorithm for 2D-NMR signals}

The 2D-NMR signal contains more information than the 1D-NMR signal. As a result, several high-resolution methods have been proposed to quantitatively analyse a 2D-NMR signal. These methods suffer from numerous numerical issues. To begin with, the presence of an extra dimension in the signal increases the volume of sampled data. This creates matrices of very large size which, for the matrix decomposition procedures required by the quantitaion methods, can be computationally prohibitive. Secondly, large polynomial rooting might be required to extract the increased resonance coupling information encapsulated in the 2D-NMR signal. Collectively, these issues impart tremendous constraints on memory and com- 
putational complexity, let alone the issues of accuracy, resolution and automation which may arise due to artefacts in the 2D peak shape caused by magnetic inhomogeneities and uncertainties in the chemical environment underlying the 2D-NMR experiment. Therefore, a fast, accurate, high-resolution and automatic 2D-NMR quantitation method is currently unavailable. A detailed overview of the start of the art in this area would be carried out in Chapter 6 .

\subsection{Further related NMR concepts}

Further NMR concepts related to the research topic are described below.

\subsubsection{Chemical shift}

The magnetic field sensed by a nucleus in an NMR experiment, in addition to the applied magnetic field, is affected by the magnetic fields of the surrounding electrons. The field "sensed" by the nucleus is equal to the applied field less the induced field. The greater the electron density surrounding a nucleus, the greater will be the induced field and vice versa. A nearby electronegative group serves to reduce this electron density and hence, the surrounding magnetic field. Consequently, such a nucleus absorbs at a lower field value and is considered as de-shielded. A nearby electropositive group will serve to increase the electron density, thereby increasing the induced field and causing the nucleus to absorb at higher fields. The reference molecule in this regard, in proton NMR, is Trimethylsilane (TMS) [176]. Absorption by any other molecule, exhibited in terms of peaks, is measured in terms of the position of these peaks relative to TMS, and is known as the chemical shift. Shielded nuclei, absorbing at higher fields, generate peaks close to TMS. These peaks, therefore, are regarded as having low chemical shifts. De-shielded nuclei, absorbing at lower fields, result in peaks far from the TMS and are regarded to have 


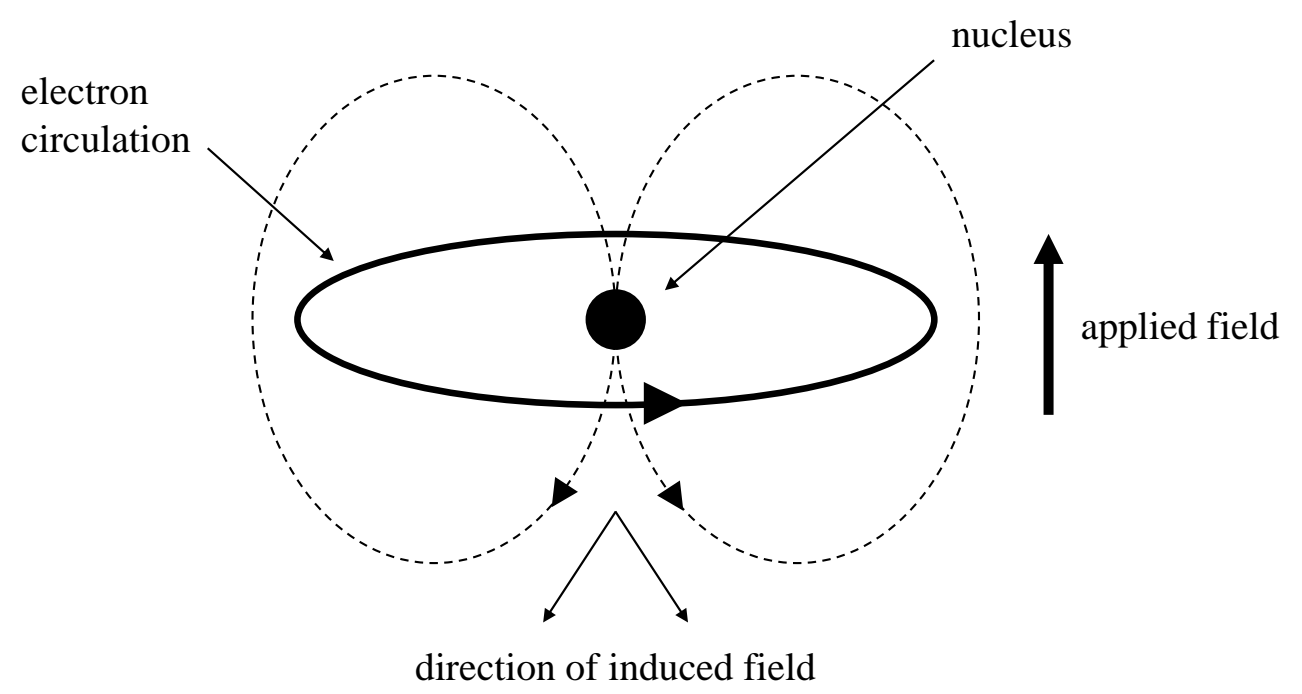

Figure 2.4: Illustration of shielding effect responsible for chemical shift [138].

higher chemical shifts.

\subsubsection{The ppm scale}

The chemical shift of a peak, measured down-field from TMS, has units of magnetic field. Using (2.5), the magnetic field and consequently, the chemical shift can be expressed in terms of the $B_{0}$ frequency. The chemical shift in $\mathrm{Hz}$ varies directly with the operating frequency. Using this ratio makes the chemical shift independent of the operating frequency of the NMR equipment.

$$
\delta=\frac{\text { chemical shift in } \mathrm{Hz}}{\text { operating frequency in } \mathrm{MHz}}
$$

$\delta$ has unit of parts-per-million (ppm). 


\subsubsection{Spin-Spin coupling}

In a given molecule, the nuclei neighbouring each other, acting as magnetic dipoles, also influence and interact with each other. Their interaction causes the peaks observed in the FID spectrum to be split in to a series of fine adjoining peaks, known in general as multiplets [176]. Presence of multiplets complicates the FID spectrum.

\subsubsection{The $n+1$ rule}

The $n+1$ rules predicts the multiplicity of peaks due to spin-spin coupling in proton NMR. The rule states that a peak in proton NMR spectrum is split into $n+1$ peaks if the number of equivalent protons in adjacent positions is $n$. The sizes of peaks in multiplets can be computed from Pascal's triangle [176].

\subsubsection{Carbon satellites}

Carbon satellites are peaks observed in the neighbourhood of peaks in ${ }^{1} \mathrm{H}$ NMR spectra [195], arising due to the spin-spin coupling of hydrogen atoms with neighbouring ${ }^{13} \mathrm{C}$ atoms. Distinguishing properties of carbon satellites include: (a) appearance of evenly spaced peaks on either side of the ${ }^{1} \mathrm{H}$ peak, and (b) splitting order being the same as that of the ${ }^{1} \mathrm{H}$ peak they surround. 


\section{Chapter 3}

\section{Parametric Estimation in NMR: New general CRLB results}

This chapter presents, in three steps, the results achieved for the first part of our work, i.e., general results on the analytical evaluation of the CramerRao Lower Bound (CRLB) for parametric estimation in NMR. Background to the problem, models of fundamental importance in this regard and the significance of the reported results are discussed first. Then a formal mathematical statement of the problem along with the methods employed to solve this problem are elaborated. Finally, the derived results are presented and their efficacy is demonstrated by application to real-time MRS data, in conjunction with the key insights gained.

\subsection{Background}

Model-based techniques are often employed to analyse the data acquired from Nuclear Magnetic Resonance (NMR) experiments for rapid quantitation and hypothesis testing [67]. For example, these techniques have been recently shown to detect the bulk constituents, i.e., water, fats and carbohydrates, in food from time-domain ${ }^{1} \mathrm{H}$ NMR (also termed as low-field NMR) data with virtually no bias [89]. Model-based techniques rely on ac- 
curate extraction of information from the recorded signal which, in turn, depends on the accuracy of an estimator employed for data processing.

The fundamental estimation theory tool for assessing the performance of an unbiased estimator is the Cramer-Rao Lower bound (CRLB). The CRLB allows quantification of the efficiency of an estimator by comparing the estimate variance achieved with the lowest possible value obtained from the bound. When the lower bound is reached, an estimator is said to be efficient [112].

For given model parameters, the CRLB is obtained from the diagonal elements of the inverse of the associated Fisher Information Matrix (FIM). Obtaining analytical CRLB expressions for model parameters can be challenging due to the requirement of symbolic inversion of the FIM.

The first attempts made to overcome this difficulty have been in two directions: (a)- the size of the FIM was kept in limits reasonable for symbolic inversion by limiting the size of the parameter vector for a given model, i.e., computing the CRLB with respect to one or two models parameters only [97,128], and (b)- asymptotic CRLB expansions were computed based on certain model-based assumptions that allowed the FIM to be diagonal which in turn made its inversion trivial [232].

A large amount of activity in these directions has been associated with the CRLB analysis of a mixture of exponentials. This model has been found useful in a wide variety of practical applications, e.g., Magnetic Resonance Spectroscopy (MRS) [216], speech processing [53], spectral estimation [159], etc. Consequently, this model and its numerous variants have been investigated for CRLB analysis, e.g., models with damped and undamped exponentials either real or complex $[122,177]$. Some general expressions for these models were obtained by [239]. The expressions involved dependence on auxiliary matrices which made their interpretation difficult.

Consequently, attempts were made to derive expressions that, though approximate, were more meaningful, demonstrating explicit dependence 
of the CRLB on the model parameters. Such expressions were reported for a few model parameters at first and later, for all free parameters, but were restrictive in their scope, e.g., they were either for frequency estimates of two closely-spaced complex damped exponential (cisoids) [207] or for the unbiased estimates of frequency, damping factor, amplitude and phase of a single cisoid only [208]. Cavassila et al. reported approximate analytical CRLB expressions for single, double, and triple cisoids [37].

The results reported by Cavassila et al. demonstrated explicit dependence of the CRLB on model parameters for singlet and doublets, but not for the triplets due to the numerous assumptions made to simplify the expressions for this case. The expressions obtained for the case of triplets were quite intricate and did not offer insight. This, once again, can be attributed to the difficulty associated with symbolic inversion of the FIM. The size of the FIM is proportional to the number of cisoids times the number of parameters to be estimated for each cisoid.

Lately, efforts have been made to avoid the inversion of entire FIM, and to obtain the Equivalent Fisher Information (EFI) instead [186]. The EFI is computed by inverting the FIM for the "parameters of interest" only. For example, the EFI for selected model parameters was reported for the source localisation problem in the near field of a sensor array [65]. The reported expressions were for one - and later two [66] - near-field sources only. Similar EFI was reported to analyze the performance of wavefield estimators in array processing of seismic waves [143]. The reported expressions were limited to the analysis of rotational or translational measurements of a single Love or Rayleigh wave.

Recently, CRLB expressions for a generalisation of the damped cisoid model, the complex quasi-polynomial model, have been reported [13]. These expressions depend on auxiliary expressions for their explanation and do not demonstrate the explicit dependence of the CRLB on model parameters; no further information is provided about the auxiliary expressions other than that they are continuous, finite and positive. This makes 
them difficult to interpret, which is problematic when intepretability was one of the key motivations of that work. For interpretation purposes, the authors provide asymptotic first-order expansions of the CRLB terms with respect to the number of signal samples - leaving out the sampling rate, frequency, and the damping factor.

The authors of [13] provide similar asymptotic first-order expansions for the damped cisoid model. This restricts their scope when compared with earlier results reported in this regard by Wigren et al. [232], which demonstrate the asymptotic dependence of the CRLB on frequency and damping factor as well. Moreover in [13], variation of the CRLB with respect to the frequency gap and the damping factor have been presented for a maximum of two cisoids only. This case, as we saw earlier, was more thoroughly addressed by Cavassila et al. [37] in their earlier work where they show the explicit dependence of the CRLB on the amplitude, phase, sampling rate, frequency, and damping factor for the damped cisoid model containing two components.

Motivated by this, we derived analytical expressions for the CRLB on the variance of unbiased estimates of frequency, damping factor and complex amplitude of an arbitrary number of damped cisoids in complex Additive White Gaussian Noise (AWGN), i.e., the Magnetic Resonance Spectroscopy (MRS) model. The expressions display the explicit relationship of the CRLB for each distinct parameter with respect to all the free model parameters. The reported expressions do not involve auxiliary expressions for their explanation and are readily interpretable; they allow the elicitation of the behaviour of the CRLB in terms of all of the free model parameters, i.e., the number of cisoids, their amplitudes, phases, frequencies, damping factors, Signal-to-Noise Ratio (SNR) and sampling rate, without imposing any restriction on any of them.

In addition to the CRLB, the results for the determinant and the condition number of the FIM for the MRS model for an arbitrary number of cisoids are also provided. These results - the CRLB from the inverse of 
the FIM, its determinant and the condition number - are also derived for the Magnetic Resonance Relaxometry (MRR) model which can be considered as a special case of the MRS model. Furthermore, these results are also presented for the MRS model with previous knowledge, which enables the incorporation of prior information about the damping factors and frequencies in the MRS model and hence, the possibility to analyse the influence of previous knowledge on the results obtained for the MRS model.

Moreover, some auxiliary results are provided for the abovementioned models, i.e., on the multiplicity and separation of eigenvalues, the magnitude of the largest and smallest eigenvalues and the factorisation of the characteristic polynomial of the associated FIMs of these models.

Finally, the efficacy of the derived results is demonstrated by their application to real-time MRS data. The derived expressions successfully explain various physical and chemical phenomena observed in realtime MRS data and their impact on estimation accuracy of a quantitation method when applied to MRS data, i.e., impact of model type, peak multiplicity, multiplet symmetry, solvent peak, carbon satellites, baseline distortion, signal truncation, and the second-order effects observed in spincoupled systems.

The chapter is organised as follows. Section 3.2 presents the models considered in this work. The motivation for the specific results derived for these model are discussed in Section 3.3. A problem statement along with the methods employed to solve the problem, i.e., the Leverrier-Faddeev method and the method of Lobachevsky-Graeffe, are outlined in Sections 3.4 and 3.5, 3.6 respectively. The results derived using these methods are presented in Section 3.7. The efficacy of these results, by their application to real-time MRS data, is demonstrated in Section 3.8. 


\subsection{Significance of the models considered}

\subsubsection{The MRS model}

The MRS model constitutes complex exponentially damped sinusoids (cisoids) embedded in complex Additive White Gaussian Noise (AWGN). The model has been considered a classic in signal processing ever since the time of Gaspard Riche de Prony who developed the well-known Prony's method for the analysis of such signals [209]. The model continues to attract significant attention as these signals keep arising in modern applications.

For example, in addition to Nuclear Magnetic Resonance (NMR), this model occurs in a myriad of other spectroscopic techniques such as Nuclear Quadrupole Resonance (NQR) [87], Electron Paramagnetic Resonance (EPR) [52], Ion Cyclotron Resonance (ICR) [45], and Microwave Resonance spectroscopy [33]. The Free Induction Decay (FID) signal measured in these techniques, as well as NMR, is modeled as a sum of exponentially damped sinusoids.

Applications of these spectroscopic techniques are far reaching: in analysis of complexes produced in a pulsed supersonic jet expansion [84], non-invasive biological and health sensing for characterisation of biomaterials [116], probing reaction mechanisms in organic matter that pyrolyzes to form petroleum [169], paramagnetic tagging of diamagnetic proteins [178], etc.

Exponentially damped sinusoidal signals also emerge in a variety of other practical applications. For example, the human heart sounds that arise from impairment of artificial heart valves have been shown to be composed of exponentially damped sinusoidal signals [183]. The oscillations that arise due to the interconnection of electrical power systems are shown to be best described by exponentially damped sinusoidal signals [215]. Voltage and current measurements at the terminals of a Pulsed Corona Reactor (PCR) - used for improving air quality - are best estimated 
by a sum of damped sinusoidal functions [188]. The dominant complex natural responses of radar targets also constitute exponentially damped oscillatory signals [43].

Furthermore, these signals are found in many other areas of interest, e.g., spectral-line estimation [159], source localisation [1], audio signal modelling and compression [53] and biomedical signal processing [223].

\subsubsection{The MRR model}

Multicomponent magnetic resonance $T_{2}$ relaxometry provides a quantitative method for the characterisation of tissues and consequently finds application in numerous branches of Magnetic Resonance Imaging (MRI) [144]. Several other practical applications that utilise multicomponent $T_{2}$ estimates are breast tissue characterisation [85], treatment of multiple sclerosis [130], and myelin quantification in the brain [23].

The MRR model constitutes real exponentials embedded in AWGN [231]. It is an important and well-studied model that, in addition to relaxometry, arises in numerous other science/engineering problems including semiconductor physics [102], transient spectroscopy [125], analysis of radioactive decays [117], reaction kinetics [22], and medical imaging [187]. No less than fifteen different areas have been identified in which MRR finds applications [105]. The problem is to determine the number of decaying exponentials as well as their amplitudes and damping factors from empirical data.

\subsubsection{The MRS model with previous knowledge}

As we saw earlier, the MRS model finds widespread use in spectroscopic techniques. Out of these techniques, NMR is emerging as one of the most recognised quantitative tools. Under the umbrella of quantitative NMR (qNMR), it is being widely exploited in a range of applications: metabolomics for complex chemical mixture analysis [229], pharmaceu- 
tical analysis for drug identification and testing [100], in food and drink industry for adulteration detection [5,120], and as well as reaction/process monitoring [24].

NMR can extract highly detailed structural information from the spectra of a sample by employing a wide variety of experimental techniques [34]. The resulting NMR spectra can contain densely overlapped peaks which makes them very complex. These peaks are often separated based on their physical properties. In the scenarios characterising a large number of spectra from a single source, prior knowledge, in the form of spectral databases or operator interaction, can be imposed for separation and elicitation of quantitative information from peaks of interest, e.g., quantitation of peak height in a mixture analysis [63].

\subsection{Problem motivation}

\subsubsection{The CRLB}

The CRLB provides a lower bound on the variance of the estimates achieved by an unbiased estimator. Analytical evaluation of the CRLB can provide explicit insight into the role of the model parameters in determining the behaviour of the estimator. This can be utilised to identify the trade-offs that characterise the sensitivity of the bound and the parameters associated with it and eventually, to optimise the performance of the estimator.

Furthermore, the insights gained from the CRLB can be employed for optimal experimental design. For example, they have been used to optimise the sampling time for the measurement of longitudinal relaxation time $T_{1}$ and the transverse relaxation time $T_{2}$ in magnetic resonance spinecho experiments [110]. The impact of model uncertainties on the CRLB has been utilised for detection of static eccentricity in synchronous machines [64]. CRLB has also been used to optimise the performance of di- 
rection of arrival estimation in antenna array configurations [227]. More recently, the CRLB has been used to optimise low SNR multi-coil acquisitions experiments in Magnetic Resonance Imaging (MRI) [27].

\subsubsection{The determinant, trace, and minimum and maximum eigenvalues}

The determinant of the inverse of the FIM (equal to the inverse of the determinant) has been used as an objective function in order to optimise the parameter estimates for a given model. This procedure, known as D-optimal design, seeks to minimise the volume of an ellipsoid representing the errors in estimates and, due to its appealing geometrical interpretation, has been largely employed for experimental design [211].

The Effective Independence Distribution (EID) method was one of the first methods employing the determinant of the FIM as an objective function. It was developed by Kammer for the selection of optimal sensor locations in a modal identification experiment on a space station [111]. Since then, the determinant of the FIM has found application in a wide variety of optimisation problems. Examples include: to minimise the parameter uncertainty of IL-6 signaling pathways for elucidation/modulation of biological responses to inflammation; to optimise receiver trajectory in scanbased emitter localisation problem for stationary pulsed radars [61]; and in path planning of a team of Uninhabited Aerial Vehicles (UAVs) for the geolocation problem [62], etc.

Analogous to the D-optimal design, the smallest eigenvalue of the FIM, the trace of the inverse of the FIM, and the trace of the FIM form the basis of E-optimal, A-optimal, and T-optimal design respectively [10,168]. These criteria have been extensively investigated for a variety of applications, e.g., for adaptive design of waveforms in MIMO radar systems [93], for infinite-dimensional regularised Bayesian inverse problems [7], and for the computation of optimal input trajectories in nonlinear dynamical sys- 
tems respectively [72].

\subsubsection{The condition number}

The FIM is frequently employed to characterise the performance of an estimator. In certain conditions, the FIM cannot accurately predict the amount of information obtained from the observations. This can happen in parameter estimation problems for high-dimensional models or for models that depend strongly on few parameters with priors for the weaker parameters not considered properly, e.g., the Stationary Phase Approximated (SPA) model for parameter estimation of gravitational waves [217].

For such models, the notion of condition number can be employed to analyse unusual conditions on the perturbation of the inverse of the FIM and hence, variations engendered in its diagonal elements, i.e., the predicted estimate variances. A large value of the condition number signifies the presence of degenerate linear combinations of parameters or the "unusual conditions" that lead to degeneracy of the FIM [200]. Analytical expressions for the condition number of the FIM can explicitly lead to the identification of such unusual conditions or parameters for a given model.

\subsection{Problem statement}

We consider minimum variance unbiased estimation of a real parameter vector $\boldsymbol{\theta}$ of size $L$ :

$$
\boldsymbol{\theta}=\left[\begin{array}{llll}
\theta_{1} & \theta_{2} & \ldots & \theta_{L}
\end{array}\right]^{T}
$$

based on the complex data vector $z$ :

$$
\boldsymbol{z}=[z(0), z(1), \ldots, z(M-1)]
$$

the $m$-th observed sample of which is $z(m)$, in the presence of complex white Gaussian noise, having zero mean and variance $\sigma^{2}$ independent of $\boldsymbol{\theta}$. The $i j$-th element of the Fisher Information Matrix (FIM) for complex 
data $\boldsymbol{z}$, parameterised by real vector $\boldsymbol{\theta}$ independent of the noise variance, is given by [112]:

$$
[\mathbf{J}]_{i j}=\frac{2}{\sigma^{2}} \Re\left[\sum_{m=0}^{M-1} \frac{\partial \tilde{z}(m)^{*}}{\partial \theta_{i}} \frac{\partial \tilde{z}(m)}{\partial \theta_{j}}\right]
$$

$\tilde{\boldsymbol{z}}$ is the noise-free version of $\boldsymbol{z}$. The CRLB is computed by inverting $\mathbf{J}$.

\subsection{The Leverrier-Faddeev method}

In this section, we explore the Leverrier-Faddeev (LF) method which enables the computation of a matrix inverse along with the coefficients of its characteristic polynomial and the eigenvectors. This method was suggested by Leverrier in 1840 [127] and modified by Faddeev in 1963 [70]. The method was rediscovered independently by Frame [75] and Souriau [194] in 1948 and 1949 respectively.

\subsubsection{Leverrier's method}

Leverrier's method enables the determination of the characteristic polynomial associated with a matrix. It is based on Newton's formulas for the sums of the powers of the roots of an algebraic equation, and aims at recursive computation of the coefficients associated with the characteristic polynomial of a matrix. Let:

$$
\phi(\lambda)=\lambda^{L}+q_{1} \lambda^{L-1}+\cdots+q_{L}
$$

be the characteristic polynomial associated with the matrix $\mathbf{J}$ such that $\phi(\lambda)=\operatorname{det}(\mathbf{J}-\lambda \mathbf{I})$, where $\mathbf{I}$ is the $L \times L$ identity matrix and $\lambda_{1}, \lambda_{2}, \ldots, \lambda_{L}$ are the of roots of polynomial $\phi(\lambda)$. Considering the sums:

$$
t_{k}=\sum_{l=1}^{L} \lambda_{l}^{k}
$$


with $k=1,2, \ldots, L$ such that:

$$
t_{k}=\lambda_{1}^{k}+\lambda_{2}^{k}+\cdots+\lambda_{L}^{k}=\operatorname{trace}\left(\mathbf{J}^{k}\right)
$$

then for $k \leq L$, the following relationship holds between $q_{k}$ and $t_{k}$ according to Newton's formula [148]:

$$
t_{k}+q_{1} t_{k-1}+\cdots+q_{k-1} t_{1}=k q_{k}
$$

$q_{k}$ can be recursively computed from $t_{k}$ using (3.7):

$$
q_{k}=-\frac{1}{k}\left(t_{k}+q_{1} t_{k-1}+\cdots+q_{k-1} t_{1}\right)
$$

with $k=1,2, \ldots, L$. It follows from (3.8) that the coefficients of the characteristic polynomial of the matrix $\mathbf{J}$ can be computed if the sums $t_{1}, t_{2}, \ldots, t_{L}$ are known. Hence, Leverrier's method for determining the coefficients $q_{k}$ of the characteristic polynomial $\phi(\lambda)$ associated with the matrix $\mathbf{J}$ consists of computing the matrix powers $\mathbf{J}^{k}$, finding the sums $t_{k}$ which are their respective matrix traces, and then employing (3.8) to calculate $q_{k}$.

\subsubsection{Modifications proposed by Faddeev}

The modification proposed by D. K. Faddeev to Leverrier's original method not only simplifies the determination of the coefficients of the characteristic polynomial of $\mathbf{J}$, but also enables the computation of its inverse and the eigenvectors. The modified Leverrier's method suggests the computation of the following sequence of matrices in place of $\mathbf{J}, \mathbf{J}^{2}, \ldots, \mathbf{J}^{L}$.

$$
\mathbf{A}_{k}=\mathbf{J B}_{k-1}
$$

with

$$
\mathbf{B}_{k}=\mathbf{A}_{k}-r_{k} \mathbf{I}
$$

and $\mathbf{I}$ being the Identity matrix, $\mathbf{A}_{1}=\mathbf{J}$ and:

$$
r_{k}=\frac{\operatorname{trace}\left(\mathbf{A}_{k}\right)}{k}
$$


The coefficients $r_{k}$ obtained in (3.11) satisfy Newton's formula in (3.7) and are related to the coefficients of $\phi(\lambda)$ in (3.8) by:

$$
r_{k}=-q_{k}
$$

In addition to the determination of the coefficients associated with the characteristic polynomial of $\mathbf{J}$, Faddeev's modification enables the computation of $\mathbf{J}^{-1}$ and the determinant of $\mathbf{J}$, which shall be demonstrated subsequently.

\subsubsection{Computation of matrix inverse}

Faddeev's modification makes it possible to compute the inverse of $\mathbf{J}$ from (3.9) and (3.10) as follows. The sequence of matrices $\mathbf{B}_{k}$ in (3.10) terminates at the $L$-th step with $\mathbf{B}_{L}=\mathbf{0}$ :

$$
\mathbf{A}_{L}-r_{L} \mathbf{I}=\mathbf{0}
$$

for which (3.9) becomes:

$$
\mathbf{A}_{L}=\mathbf{J B}_{L-1}
$$

which when substituted in (3.13) leads to:

$$
\mathbf{J}^{-1}=\frac{\mathbf{B}_{L-1}}{r_{L}}
$$

\subsubsection{Computation of determinant}

The determinant of the FIM can be found from (3.4). For this, we consider Vieta's expansion [71] of polynomials which relates the coefficients of a polynomial to the product and sums of its roots. Allowing $\lambda_{1}, \lambda_{2}, \ldots, \lambda_{L}$ to be the roots of characteristic polynomial $\phi(\lambda)$, (3.4) can be written as:

$$
\phi(\lambda)=\left(\lambda-\lambda_{1}\right)\left(\lambda-\lambda_{2}\right) \ldots\left(\lambda-\lambda_{L}\right)
$$


Expanding binomials in (3.16) and equating them with the coefficients of identical powers in (3.4) leads to the following relationships between the roots and coefficients of $\phi(\lambda)$ :

$$
\begin{aligned}
\lambda_{1}+\lambda_{2}+\cdots+\lambda_{L} & =-q_{1} \\
\lambda_{1} \lambda_{2}+\lambda_{1} \lambda_{2}+\cdots+\lambda_{L-1} \lambda_{L} & =q_{2} \\
\vdots & \\
\lambda_{1} \lambda_{2} \ldots \lambda_{L} & =q_{L}
\end{aligned}
$$

The left-hand side of (3.17) represents the sums of products of roots of $\phi(\lambda)$ taken in $k$-tuples. From the fundamental theorem of linear algebra, the product of the roots of the characteristic polynomial - alternatively the eigenvalues of the associated matrix - is equal to the determinant of the matrix [205]. This product we note is $q_{L}$ in (3.17). From (3.11), the result follows:

$$
r_{L}=-\lambda_{1} \lambda_{2} \ldots \lambda_{L}
$$

which equals the negative of the determinant of J. Hence:

$$
\operatorname{det}(\mathbf{J})=-r_{L}
$$

\subsubsection{Computation of eigenvalues}

Though the LF method allows the determination of the characteristic polynomial, the inverse and the determinant of $\mathbf{J}$ simultaneously, no mention was made regarding the computation of the eigenvalues of $\mathbf{J}$, i.e., the roots of the characteristic polynomial $\phi(\lambda)$. This, if performed symbolically by the traditional rooting process, can be a tedious task for polynomials of high degree. The Abel-Ruffini theorem [2] states that for a polynomial of degree greater than or equal to five, no formula can be found which would express the roots of such a polynomial by its coefficients in terms of radicals. Therefore, we resort to an approximate method for the calculation of the roots of the characteristic polynomial $\phi(\lambda)$, i.e., the method of Lobachevsky and Graeffe [54]. 


\subsection{The Lobachevsky-Graeffe method}

Once the characteristic polynomial of the matrix $\mathbf{J}$ is determined using the LF method, the roots of (3.4) or equivalently, the eigenvalues of $\mathbf{J}$, can be found by the help of the Lobachevsky-Graeffe (LG) Method.

\subsubsection{Basic principle}

In order to illustrate the basic principle behind the method, we reconsider (3.4) and assume that its roots $\lambda_{k}$ are such that:

$$
\left|\lambda_{1}\right| \gg\left|\lambda_{2}\right| \gg \cdots \gg\left|\lambda_{L}\right|
$$

with $\lambda_{1}$ and $\lambda_{L}$ being the largest and the smallest roots of $\phi(\lambda)$ respectively, and each root in (3.20) may have multiplicity greater than one. The roots are distinct in modulus, and the ratio of the moduli of any two successive roots is a small quantity:

$$
\left|\lambda_{k}\right|=\epsilon_{k-1}\left|\lambda_{k-1}\right|
$$

with $\left|\epsilon_{k}\right|<\epsilon \forall k, \epsilon$ being a small number, i.e., the roots are well-separated. From Vieta's expansions in (3.17), and by virtue of (3.21), we get:

$$
\begin{aligned}
\lambda_{1}\left(1+\xi_{1}\right) & =-q_{1} \\
\lambda_{1} \lambda_{2}\left(1+\xi_{2}\right) & =q_{2} \\
\vdots & \\
\lambda_{1} \lambda_{2} \ldots \lambda_{L}\left(1+\xi_{L}\right) & =q_{L}
\end{aligned}
$$

such that the quantities $\left|\xi_{k}\right| \ll 1$. Neglecting $\xi_{k}$ in (3.22) results in the following approximate relations:

$$
\begin{aligned}
\lambda_{1} & \approx-q_{1} \\
\lambda_{1} \lambda_{2} & \approx q_{2} \\
\vdots & \\
\lambda_{1} \lambda_{2} \ldots \lambda_{L} & \approx q_{L}
\end{aligned}
$$


whence we find the desired roots:

$$
\lambda_{k} \approx-\frac{q_{k}}{q_{k-1}}
$$

with $k=1,2, \ldots, L$ and $q_{0}=1$.

\subsubsection{The root separation process}

The underlying principle behind the LG method can be garnered from the examination of (3.24). The LG method states that if the roots of the characteristic polynomial $\phi(\lambda)$ are well-separated, they can be approximately determined by the following system of linear equations:

$$
\begin{aligned}
\lambda_{1}+q_{1} & =0 \\
q_{1} \lambda_{1}+q_{2} & =0 \\
\vdots & \\
q_{L-1} \lambda_{1}+q_{L} & =0
\end{aligned}
$$

In order to meet the requirement of well-separation of roots, the following transformation of (3.4) was proposed by Lobachevsky [20]:

$$
\mu^{L}+q_{1}^{\kappa} \mu^{L-1}+\cdots+q_{L}^{\kappa}=0
$$

such that the roots $\mu_{1}, \mu_{2}, \ldots, \mu_{L}$ of (3.26) are the $\kappa$-th powers of the roots $\lambda_{1}, \lambda_{2}, \ldots, \lambda_{L}$ of $\phi(\lambda)$ :

$$
\mu_{k}=\lambda_{k}^{\kappa}
$$

If the value of $\kappa$ is sufficiently large, the roots of (3.26) will be wellseparated:

$$
\lim _{\kappa \rightarrow \infty} \frac{\mu_{k}}{\mu_{k-1}}=\left(\frac{\lambda_{k}}{\lambda_{k-1}}\right)^{\kappa} \rightarrow 0
$$

This transformation can be achieved by repeatedly performing the wellknow Lobachevsky-Graeffe root-squaring process [20]. In order to illustrate the idea behind this process, we reconsider the characteristic polynomial $\phi(\lambda)$ in (3.16) and substitute $-\lambda$ in place of $\lambda$ :

$$
\phi(-\lambda)=\left(\lambda+\lambda_{1}\right)\left(\lambda+\lambda_{2}\right) \ldots\left(\lambda+\lambda_{L}\right)
$$


Now multiplying (3.29) by (3.16):

$$
\phi(\lambda) \phi(-\lambda)=\left(\lambda^{2}-\lambda_{1}^{2}\right)\left(\lambda^{2}-\lambda_{2}^{2}\right) \ldots\left(\lambda^{2}-\lambda_{L}^{2}\right)
$$

and setting $\mu=-\lambda^{2}$ we obtain:

$$
\varphi(\mu)=\left(\mu+\lambda_{1}^{2}\right)\left(\mu+\lambda_{2}^{2}\right) \ldots\left(\mu+\lambda_{L}^{2}\right)
$$

We observe that the roots of the resulting polynomial $\varphi(\mu)$ are the square of the roots of the original polynomial $\phi(\lambda)$ :

$$
\mu_{k}=-\lambda_{k}^{2}
$$

Now we expand (3.31):

$$
\varphi(\mu)=\mu^{L}+s_{1} \mu^{L-1}+\cdots+s_{L}
$$

and employ the LG method to determine its coefficients $s_{1}, s_{2}, \ldots, s_{L}$ from the following system of linear equations:

$$
\begin{aligned}
\mu_{1}+s_{1} & =0 \\
s_{1} \mu_{1}+s_{2} & =0 \\
\vdots & \\
s_{L-1} \mu_{1}+s_{L} & =0
\end{aligned}
$$

such that:

$$
\mu_{k}=-\frac{s_{k}}{s_{k-1}}
$$

Considering the relation in (3.32), we obtain:

$$
\lambda_{k}= \pm \sqrt{-\mu_{k}}=\sqrt{\frac{s_{k}}{s_{k-1}}}
$$

for $k=1,2, \ldots, L$. With these results we observe that the principal requirement of the LG method, i.e., the root isolation, can be met by applying the root squaring process. However, the pursuit of symbolic results can complicate an already unwieldy polynomial by requiring its coefficients to be squared. This difficulty can be circumvented by considering the work of Hua [115] which concerns polynomials having real coefficients and real roots - both of which are valid in this case. 


\subsubsection{Hua's theorem}

Hua's theorem [115] states that given the coefficients of a polynomial are real (which immediately follows from (3.3)) and all its roots are real (this again follows from the fact that $\mathbf{J}=\mathbf{J}^{T}$ ) then the square of each nonextreme coefficient of the polynomial $\phi(\lambda)$ is greater than the product of two adjacent coefficients, i.e., for (3.4):

$$
q_{k}^{2} \gg q_{k-1} q_{k+1}
$$

for $k=1,2, \ldots, L-1$. To view the implication of Hua's theorem, we determine the relationship between the transformed coefficients and the original coefficients after the root squaring process, i.e., between $s_{k}$ of $\varphi(\mu)$ in (3.33) and $q_{k}$ of $\phi(\lambda)$ in (3.4). For this, we expand the left-hand side of (3.33) in terms of (3.4) and, keeping in view the relation obtained between $\mu$ and $\lambda$ in (3.32), equate the coefficients of both sides with identical powers to obtain:

$$
\begin{aligned}
& s_{1}=q_{1}^{2}-2 q_{2} \\
& s_{2}=q_{2}^{2}-2 q_{1} q_{3}+2 q_{4} \\
& \vdots \\
& s_{L}=q_{L}^{2}
\end{aligned}
$$

The above relationship between $s_{k}$ and $q_{k}$ can be compactly expressed as:

$$
s_{k}=q_{k}^{2}+2 \sum_{l=1}^{k}(-1)^{l} q_{k-l} q_{k+l}
$$

with $q_{0}=1, q_{l}=0$ for $l<0$ and $l>L$, and $k=0,1, \ldots, L$. Hua's theorem expresses this relationship as inequality in (3.37) which, given that $q_{0}=1$, leads to:

$$
s_{k} \approx q_{k}^{2}
$$

which in conjunction with (3.36), results in:

$$
\lambda_{k} \approx \sqrt{\frac{s_{k}}{s_{k-1}}} \approx \frac{q_{k}}{q_{k-1}}
$$


If the root-squaring processing is performed an arbitrary number of times, say $\kappa$-times, a similar argument from Hua's theorem would follow at each step, leading to the following relationship between the roots of the transformed equation and the original one:

$$
\mu_{k} \approx q_{k}^{2^{\kappa}}
$$

eventually becoming:

$$
\lambda_{k} \approx \sqrt[2^{\kappa}]{\frac{\mu_{k}}{\mu_{k-1}}} \approx \frac{q_{k}}{q_{k-1}}
$$

with $k=1,2, \ldots, L$ and $q_{0}=1$. Hence, though the LG method for the approximate calculation of roots requires them to be well-separated, this requirement does not effect the computation of roots for the given problem as a consequence of Hua's theorem which, in turn, is invoked due to the symmetry of $\mathbf{J}$ and the nature of its definition in (3.3).

\subsubsection{Computation of largest eigenvalue}

The LG method begins by arranging the roots $\lambda_{k}$ of $\phi(\lambda)$ in descending order in (3.20), with $\lambda_{1}$ being the largest and $\lambda_{L}$ being the smallest root, given that each root is counted according to its multiplicity. From (3.24), we obtain the largest polynomial root of $\phi(\lambda)$ by setting $k=1$ :

$$
\lambda_{1} \approx-q_{1}
$$

with $q_{0}=1$. (3.44), in conjunction with Vieta's expansions for $k=1$ in (3.17), results in:

$$
\lambda_{1} \approx \operatorname{trace}\left(\mathbf{A}_{1}\right)
$$

Given that $\mathbf{A}_{1}=\mathbf{J}$ in (3.9), this leads to the following relationship for the largest eigenvalue $\lambda_{\max }$ of $\mathbf{J}$ :

$$
\lambda_{\max }(\mathbf{J}) \approx \operatorname{trace}(\mathbf{J})
$$




\subsubsection{Computation of smallest eigenvalue}

From elementary linear algebra, we know that the eigenvalues of the inverse of a matrix equal the inverse of the eigenvalues of the original matrix [205]. This leads to the smallest eigenvalue of $\mathbf{J}$ being the largest eigenvalue of $\mathbf{J}^{-1}$. This eigenvalue can be found by following a similar argument for $\mathbf{J}^{-1}$ as the one developed in the preceding section for $\mathbf{J}$ :

$$
\lambda_{1}^{\prime} \approx \operatorname{trace}\left(\mathbf{J}^{-1}\right)
$$

where $\lambda_{1}^{\prime}$ is the largest root of the characteristic polynomial associate with $\mathbf{J}^{-1}$. Hence, the smallest eigenvalue $\lambda_{\min }$ of $\mathbf{J}$ will be the reciprocal of $\lambda_{1}^{\prime}$ :

$$
\lambda_{\min }(\mathbf{J}) \approx \frac{1}{\operatorname{trace}\left(\mathbf{J}^{-1}\right)}
$$

$\mathbf{J}^{-1}$ can be found from our earlier discussion in Section 3.5.3 on the computation of the matrix inverse using the LF method.

\subsubsection{Computation of condition number}

From the smallest and largest eigenvalues, the condition number of $\mathbf{J}$ can be computed as [206]:

$$
\operatorname{cond}(\mathbf{J})=\frac{\left|\lambda_{\max }(\mathbf{J})\right|}{\left|\lambda_{\min }(\mathbf{J})\right|}
$$

Employing (3.46) and (3.48), cond(J) can be re-written as:

$$
\operatorname{cond}(\mathbf{J}) \approx \operatorname{trace}(\mathbf{J}) \operatorname{trace}\left(\mathbf{J}^{-1}\right)
$$

In (3.50), the modulus sign is not placed on $\mathbf{J}$, because by its definition in (3.3), $\mathrm{J}$ is a positive-definite matrix. Consequently, all of its eigenvalues are positive and hence, the trace is also positive. 


\subsection{Results}

We now present, for the models discussed in Section 3.2, the results obtained for the Cramer-Rao Lower Bound (CRLB) from the diagonal elements of $\mathbf{J}^{-1}$, for the determinant, and for the condition number.

\subsubsection{The MRS model}

In MRS, the observed signal $z$ is modelled as a sum of $N$ damped cisoids, being observed as $M$ samples, regularly spaced in time by $\delta$ :

$$
z(m)=\sum_{n=1}^{N} \beta_{n} e^{\left(-\alpha_{n}+j \omega_{n}\right) m \delta}+v(m)
$$

where $j=\sqrt{-1}$ and $\beta_{n}=x_{n}+j y_{n}$ is the complex amplitude of the $n$-th component. $\alpha_{n}>0$ and $\omega_{n}$ are its decay rate and frequency, respectively. The noise process $\boldsymbol{v}$, i.e., $\boldsymbol{v}=[v(0), v(1), \ldots, v(M-1)]$, is assumed white and complex normal, having zero mean and variance $\sigma^{2}$. The noise-free equivalent of (3.51) is $\tilde{z}(m)=\sum_{n=1}^{N} \beta_{n} e^{\left(-\alpha_{n}+j \omega_{n}\right) m \delta}$.

\section{The FIM}

The parameter vector $\boldsymbol{\theta}$ for the signal model (3.51) is defined as:

$$
\boldsymbol{\theta}=\left[\begin{array}{lllllll}
x_{1} & y_{1} & \alpha_{1} & \omega_{1} & x_{2} & \ldots & \omega_{N}
\end{array}\right]^{T}
$$

The derivatives of $\tilde{z}(m)$ with respect to the elements $\theta_{i}$ of $\boldsymbol{\theta}$ are given by:

$$
\begin{aligned}
& \frac{\partial \tilde{z}(m)}{\partial x_{n}}=p_{n}^{m} \\
& \frac{\partial \tilde{z}(m)}{\partial y_{n}}=j p_{n}^{m} \\
& \frac{\partial \tilde{z}(m)}{\partial \alpha_{n}}=-\beta_{n} m \delta p_{n}^{m} \\
& \frac{\partial \tilde{z}(m)}{\partial \omega_{n}}=j \beta_{n} m \delta p_{n}^{m}
\end{aligned}
$$


where $p_{n}=e^{\left(-\alpha_{n}+j \omega_{n}\right) \delta}$. The $i j$-th element of the FIM for complex data $\boldsymbol{z}$, parameterised by real vector $\boldsymbol{\theta}$ independent of noise variance $\sigma^{2}$, is expressed by (3.3) which allows the FIM to be expressed as [232]:

$$
\mathbf{J}=\frac{2}{\sigma^{2}}\left[\begin{array}{cccc}
\mathbf{T}^{11} & \mathbf{T}^{12} & \ldots & \mathbf{T}^{1 N} \\
\mathbf{T}^{21} & \mathbf{T}^{22} & \ldots & \mathbf{T}^{2 N} \\
\vdots & \vdots & \ddots & \vdots \\
\mathbf{T}^{N 1} & \mathbf{T}^{N 2} & \ldots & \mathbf{T}^{N N}
\end{array}\right]
$$

With four unknowns for $N$ damped cisoids each, the dimensions of $\mathbf{J}$ are $4 N \times 4 N . \mathbf{T}^{k l}$ are $4 \times 4$ block matrices resulting from (3.53) the elements of which correspond to the $k$-th and $l$-th cisoids, e.g., $T_{11}^{k l}=$ $\operatorname{Re}\left[\sum_{m=0}^{M-1} \frac{\partial \tilde{z}(m)^{*}}{\partial x_{k}} \frac{\partial \tilde{z}(m)}{\partial x_{l}}\right]$.

\section{Properties of the FIM}

From (3.54), it follows that $\mathbf{J}$ is symmetric, i.e., $\mathbf{J}=\mathbf{J}^{T}$. This symmetry also holds for its diagonal and off-diagonal blocks: $\mathbf{T}^{k k}=\left(\mathbf{T}^{k k}\right)^{T}$ and $\mathbf{T}^{l k}=$ $\left(\mathbf{T}^{k l}\right)^{T}$. The relationships among individual elements of $\mathbf{T}^{k k}$ and $\mathbf{T}^{k l}$ are depicted in Table 3.1. For each diagonal block matrix $\mathbf{T}^{k k}$, there are only four distinct elements and for each off-diagonal block $\mathbf{T}^{k l}$, there are only eight. Hence, for a FIM of size $4 N \times 4 N$, only $4 N^{2}$ elements are distinct out of its $16 N^{2}$ elements.

\section{Properties of signal model}

The sampling rate $1 / \delta$ in (3.51) is assumed sufficient (i.e., $\delta$ is small) for all frequency components to be adequately represented in the sampled signal. $M$ is also assumed large, i.e., $e^{-M \delta \alpha_{n}} \approx 0 \forall n$. This condition ensures that the signal is sampled until it has faded away, and is equivalent to ensuring that nearly all of the signal energy has been captured. This allows one to take $M \rightarrow \infty$ in the upper limit of summation in (3.3) and, in 
Table 3.1: Relationships between elements of the Fisher Information Matrix.

\begin{tabular}{ll}
\hline${\text { Elements of } \mathbf{T}^{k k}}^{k k}$ & Elements of $\mathbf{T}^{k l}$ \\
\hline$T_{12}^{k k}=0$ & $T_{11}^{k l}=T_{22}^{k l}$ \\
$T_{21}^{k k}=0$ & $T_{12}^{k l}=-T_{k l}^{21}$ \\
$T_{34}^{k k}=0$ & $T_{13}^{k l}=-T_{k l}^{24}$ \\
$T_{43}^{k k}=0$ & $T_{14}^{k l}=T_{k l}^{23}$ \\
$T_{11}^{k k}=T_{22}^{k k}$ & $T_{33}^{k l}=T_{k l}^{44}$ \\
$T_{33}^{k k}=T_{44}^{k k}$ & $T_{31}^{k l}=-T_{k l}^{42}$ \\
$T_{14}^{k k}=T_{23}^{k k}$ & $T_{32}^{k l}=T_{k l}^{41}$ \\
$T_{24}^{k k}=-T_{13}^{k k}$ & $T_{34}^{k l}=-T_{k l}^{43}$ \\
\hline
\end{tabular}

conjunction with (3.53), leads to expressions of the form [4]:

$$
\begin{gathered}
\sum_{m=0}^{\infty} p_{n}^{m}=\frac{1}{\left(1-p_{n}\right)} \\
\sum_{m=0}^{\infty} m p_{n}^{m}=\frac{p_{n}}{\left(1-p_{n}\right)^{2}} \\
\sum_{m=0}^{\infty} m^{2} p_{n}^{m}=\frac{p_{n}\left(1+p_{n}\right)}{\left(1-p_{n}\right)^{3}}
\end{gathered}
$$

with $q=0,1$, or 2 . Letting $\delta \rightarrow 0$ and $M \rightarrow \infty$, the following integral formulation was proposed for (3.55) by [37].

$$
\int_{0}^{\infty} t^{q} e^{(-\alpha+j \omega) t} d t=\frac{q !}{(\alpha-j \omega)^{q+1}}
$$

\section{The CRLB}

The following bounds are obtained from the diagonal elements of $\mathbf{J}^{-1}$ by application of (3.9), (3.10) and (3.11) of Section 3.5.2 and then (3.15) of Section 3.5.3. For $N=1$ :

$$
\sigma_{x}^{2}=\sigma_{y}^{2} \geq \delta \sigma^{2} 2 \alpha
$$


and

$$
\sigma_{\alpha}^{2}=\sigma_{\omega}^{2} \geq \frac{\delta \sigma^{2}}{a^{2}} 4 \alpha^{3}
$$

For $N=2$ :

$$
\sigma_{x_{k}}^{2}=\sigma_{y_{k}}^{2} \geq \delta \sigma^{2} 2 \alpha_{k} \varrho_{2}
$$

and

$$
\sigma_{\alpha_{k}}^{2}=\sigma_{\omega_{k}}^{2} \geq \frac{\delta \sigma^{2}}{a_{k}^{2}} 4 \alpha_{k}^{3} \rho_{2}^{2}
$$

with

$$
\varrho_{2}=\frac{\left\{\left(\alpha_{1}+\alpha_{2}\right)^{2}+\omega_{12}^{2}\right\}\left\{\alpha_{1}^{4}-8 \alpha_{1}^{3} \alpha_{2}+2 \alpha_{1}^{2}\left(15 \alpha_{2}^{2}+\omega_{12}^{2}\right)+8 \alpha_{1} \alpha_{2} \eta_{12}+\eta_{12}^{2}\right\}}{\left\{\left(\alpha_{1}-\alpha_{2}\right)^{2}+\omega_{12}^{2}\right\}^{3}}
$$

and

$$
\rho_{2}=\frac{\left(\alpha_{1}+\alpha_{2}\right)^{2}+\omega_{12}^{2}}{\left(\alpha_{1}-\alpha_{2}\right)^{2}+\omega_{12}^{2}}
$$

where $\omega_{12}=\omega_{1}-\omega_{2}$ and $\eta_{12}=\alpha_{2}^{2}+\omega_{12}^{2}$.

For arbitrary $N$ :

$$
\sigma_{x_{k}}^{2}=\sigma_{y_{k}}^{2} \geq \delta \sigma^{2} 2 \alpha_{k} \varrho_{N}^{(k)}
$$

and

$$
\sigma_{\alpha_{k}}^{2}=\sigma_{\omega_{k}}^{2} \geq \frac{\delta \sigma^{2}}{a_{k}^{2}} 4 \alpha_{k}^{3}\left\{\rho_{N}^{(k)}\right\}^{2}
$$

with

$$
\varrho_{N}^{(k)}=\prod_{\substack{l=1 \\ l \neq k}}^{N} \frac{\left\{\left(\alpha_{k}+\alpha_{l}\right)^{2}+\omega_{k l}^{2}\right\}\left\{\alpha_{k}^{4}-8 \alpha_{k}^{3} \alpha_{l}+2 \alpha_{k}^{2}\left(15 \alpha_{l}^{2}+\omega_{k l}^{2}\right)+8 \alpha_{k} \alpha_{l} \eta_{k l}+\eta_{k l}^{2}\right\}}{\left\{\left(\alpha_{k}-\alpha_{l}\right)^{2}+\omega_{k l}^{2}\right\}^{3}}
$$

and

$$
\rho_{N}^{(k)}=\prod_{\substack{l=1 \\ l \neq k}}^{N} \frac{\left(\alpha_{k}+\alpha_{l}\right)^{2}+\omega_{k l}^{2}}{\left(\alpha_{k}-\alpha_{l}\right)^{2}+\omega_{k l}^{2}}
$$

where $\omega_{k l}=\omega_{k}-\omega_{l}$ and $\eta_{k l}=\alpha_{l}^{2}+\omega_{k l}^{2}$. CRLB expressions in (3.63) and (3.64) immediately render multiple observations. To begin with, the bounds on the real and imaginary amplitudes of a cisoid are identical, and the 
bounds on the damping factor and frequency are also identical. Secondly, the bound on the amplitude of a cisoid is independent of its amplitude whereas the bound on its damping factor and frequency is inversely proportional to the cisoid amplitude, i.e., an increase in the Signal-to-Noise Ratio (SNR) will serve to improve the estimation of its damping factor and frequency only (although clearly the estimation of the amplitude expressed as a proportion of that amplitude improves). Thirdly, both bound terms (3.63) and (3.64) are directly proportional to the damping factor of a given cisoid; this demonstrates an increased difficulty associated with the estimation of rapidly decaying cisoids. The results are as expected from what is known in the literature

Fourthly, if, for the moment, we set aside bound parameters common to all terms in (3.63) and (3.64) $\left(\delta, \sigma^{2}\right.$ and the scalars), then the CRLB expressions (3.63) and (3.64) can be factored into two distinct terms: a local term $\left(\alpha_{k}\right.$ and $\alpha_{k}^{3}$ in (3.63) and (3.64) respectively), which depends on the local properties of the cisoid of interest, i.e., $\alpha_{k}$, and an interaction term $\left(\varrho_{N}^{(k)}\right.$ and $\rho_{N}^{(k)}$ in (3.65) and (3.66) respectively), which depends on the damping factor and frequency spacing of the ciosid with respect to all other cisoids, and encapsulates the impact of every other cisoid in the signal on the cisoid of interest; note here that the interaction terms depend only on the damping factor and frequency of all other cisoids, and not on their amplitudes. The significance of this observation will be discussed in detail in Section 3.8 where the interaction terms (3.65) and (3.66) will be applied to real-time MRS data.

Fifthly, we note that the interaction terms $\varrho_{N}^{(k)}$ and $\rho_{N}^{(k)}$ are not identical. Particularly, the interaction term for the amplitude bound $\varrho_{N}^{(k)}$ displays a much more complex interaction of a given cisoid with other cisoids when compared with the interaction term for the damping factor and frequency $\rho_{N}^{(k)}$.

To gain an understanding of the impact of this, the interaction terms for the case of two cisoids, i.e., $\varrho_{2}$ and $\rho_{2}$ in (3.61) and (3.62) respectively, 
are plotted individually in Figures 3.1 and 3.2 respectively, as a function of frequency spacing $\omega_{12}$ between the two cisoids and the ratio of their damping factors $k$, with $\alpha_{1}=k \alpha_{2}$. To ensure a meaningful comparison, the results in Figure 3.2 are displayed for $\rho_{2}^{2}$, this being its magnitude appearing in (3.60). We observe from Figures 3.1 and 3.2 that both $\varrho_{2}$ and $\rho_{2}^{2}$ exhibit peaks when either $\alpha_{1} \rightarrow \alpha_{2}$ or $\omega_{1} \rightarrow \omega_{2}$. This signifies an increase in the bound, and hence an increase in uncertainty associated with parameter estimation, when the damping factors or the frequencies of cisoids overlap. A singularity in the bounds is reached only when both $\omega_{1}=\omega_{2}$ and $\alpha_{1}=\alpha_{2}$. This provides yet another interesting insight that even if two cisoids overlap exactly in frequency domain, i.e., $\omega_{1}=\omega_{2}$, they can still be separately estimated if their damping factors are unique, and vice versa.

To gain further understating of the behaviour of the interaction terms, independent overlaid plots of $\varrho_{2}$ and $\rho_{2}^{2}$, as a function of $k\left(\alpha_{1}=k \alpha_{2}\right)$ and $\omega_{12}$, are displayed in Figures 3.3 and 3.4 respectively. From Figure 3.3, we observe that both $\varrho_{2}$ and $\rho_{2}^{2}$ are asymmetric with respect to $k$, i.e., the tails being heavier for $k>5$. This implies that given two cisoids have constant damping factor separation, a higher uncertainty will be associated with the estimation of their parameters if the values of their individual damping factors are large. This however is not observed for estimation of frequencies in Figure 3.4 as both $\varrho_{2}$ and $\rho_{2}^{2}$ are symmetric with respect to $\omega_{12}$. Also, we note in Figure 3.4 that the magnitude of $\rho_{2}^{2}$ is lower than that of $\varrho_{2}$ for all values of $\omega_{12}$. This, at first, might imply that the uncertainty associated with the estimation of the damping factor and frequency of a cisoid is always lower than that of its amplitude. However, from Figure 3.3, which depicts $\rho_{2}^{2}$ and $\varrho_{2}$ as a function of $k$, we observe that this is not always the case, i.e., the magnitude of $\rho_{2}^{2}$ exceeds that of $\varrho_{2}$ for $k>6$. Further, the uncertainty associated with the estimation of parameters of a cisoid, in addition to $\rho_{2}^{2}$ and $\varrho_{2}$, also depends on local terms $\alpha_{k}$ in $\alpha_{k}^{3}$ in (3.63) and (3.64) respectively.

To gain a clear understanding of the overall uncertainty associated 
with estimation of the damping factor and frequency of a cisoid and amplitude, we perform a point-by-point comparison of (3.60) and (3.59), and highlight the regions where the former exceeds the latter. The results displayed in Figure 3.5 indicate a significant region where the damping factor and frequency bound (3.60) is higher than the the amplitude bound (3.59). This is contrary to the earlier results reported in [232] which state that uncertainty associated with the estimation of damping factor and frequency is always less than that associated with the amplitude.

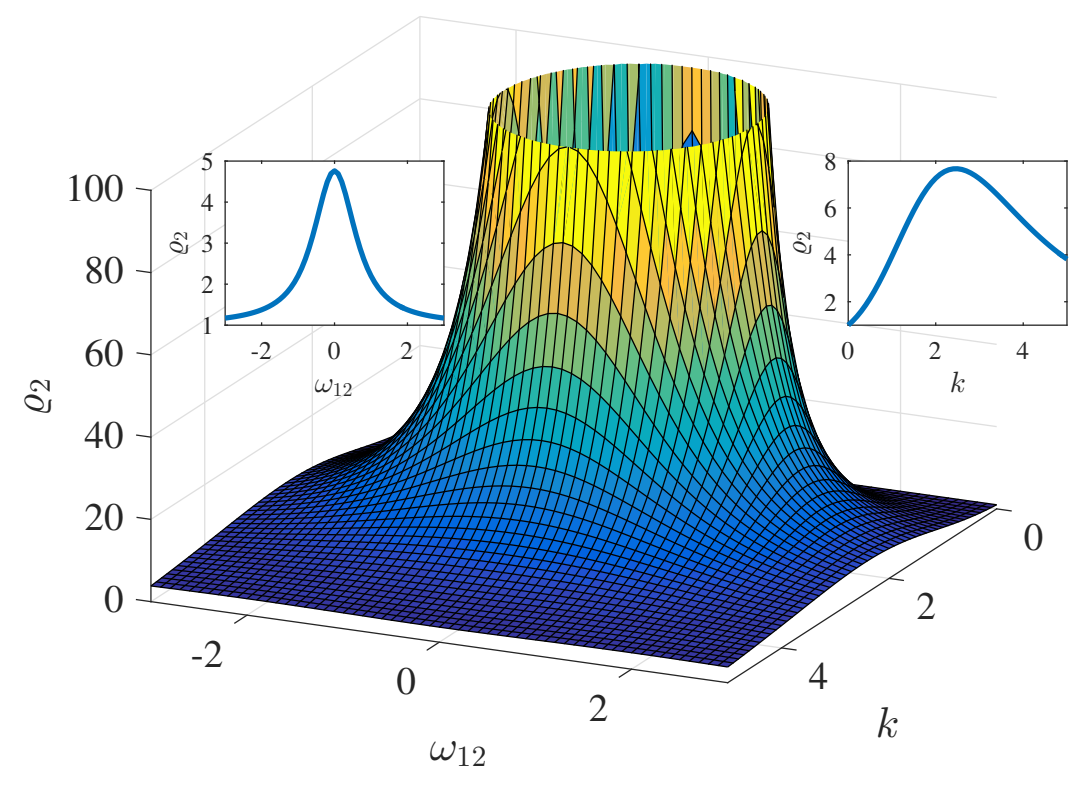

Figure 3.1: Amplitude interaction term (3.61) as a function of frequency separation $\omega_{12}$ and $k\left(\alpha_{1}=k \alpha_{2}\right)$ for the MRS model.

\section{The determinant}

The sequence of matrices obtained in (3.9) during the computation of $\mathbf{J}^{-1}$ in Section 3.5.2 enables the computation of (3.11) and subsequently, the determinant of the FIM associated with the MRS model using (3.19) of 


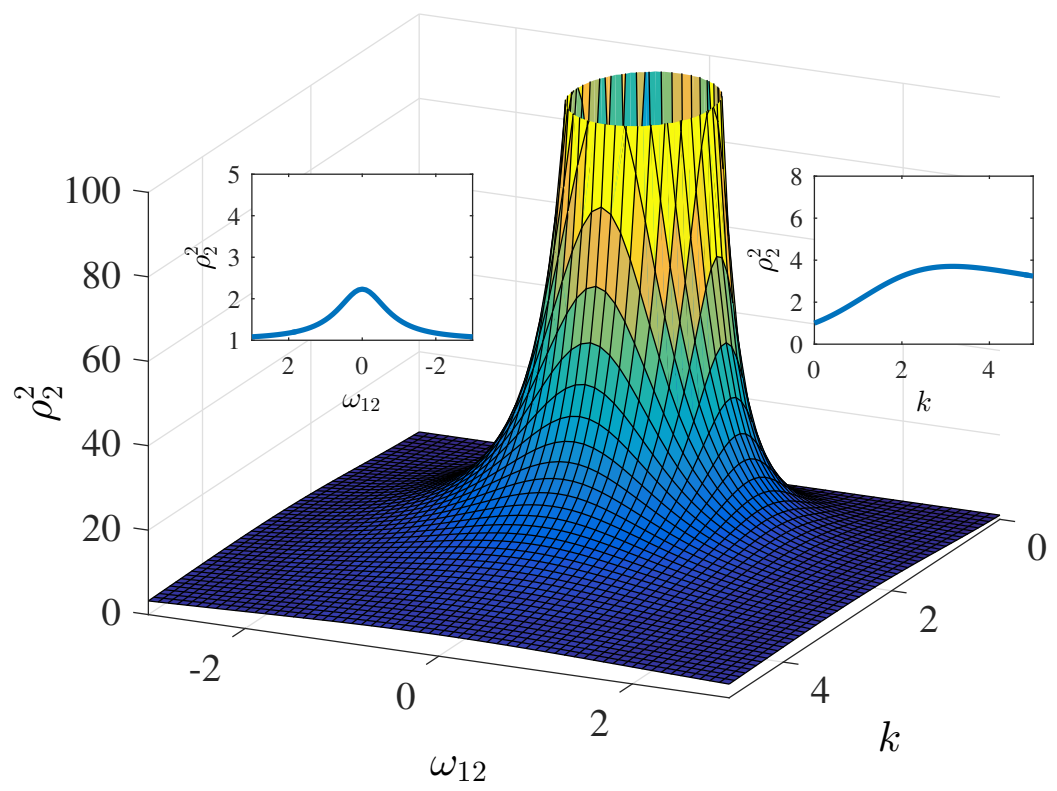

Figure 3.2: Damping factor and frequency interaction term (3.62) as a function of $\omega_{12}$ and $k\left(\alpha_{1}=k \alpha_{2}\right)$ for the MRS model.

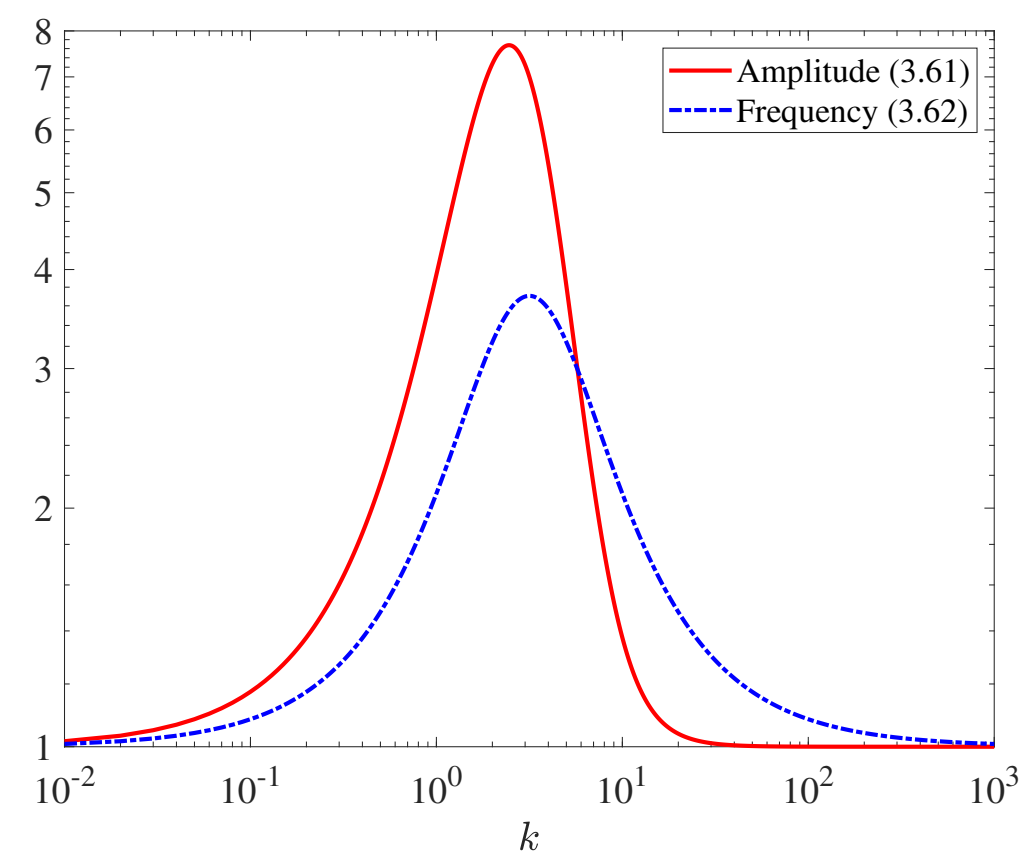

Figure 3.3: Logarithmic plot of amplitude and damping factor and frequency interaction terms (3.61) and (3.62) as a function of $k\left(\alpha_{1}=k \alpha_{2}\right)$ for the MRS model. 


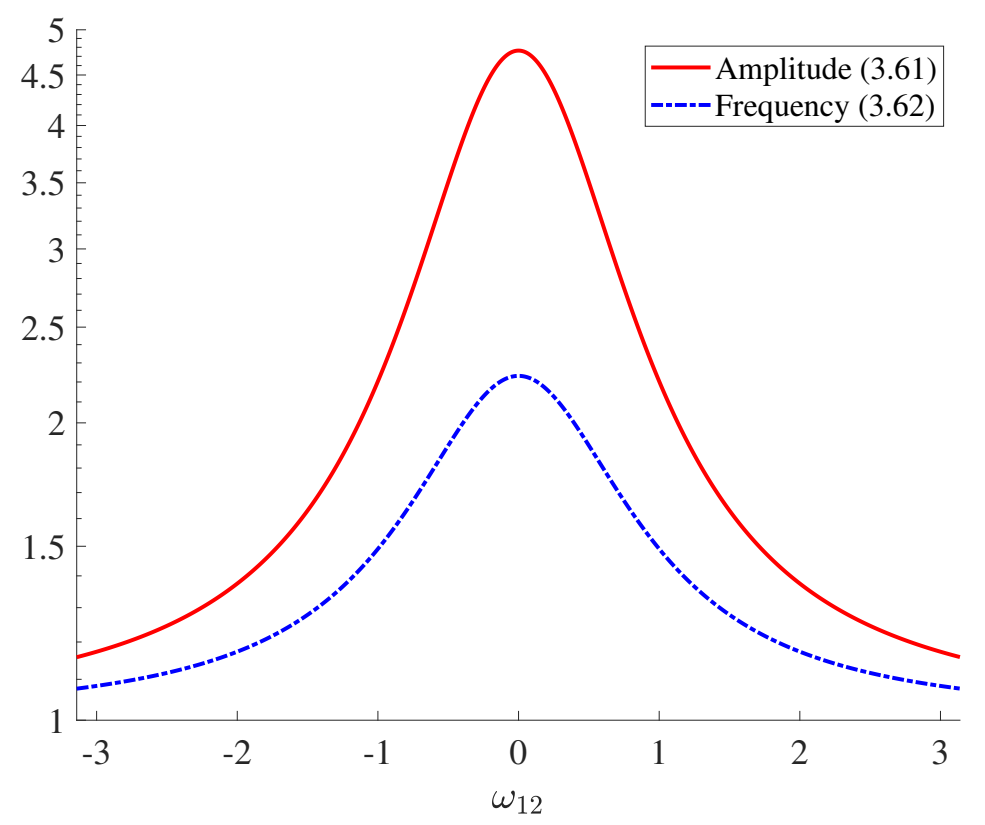

Figure 3.4: Logarithmic magnitude plot of amplitude and damping factor and frequency interaction terms (3.61) and (3.62) as a function of $\omega_{12}$ for the MRS model.

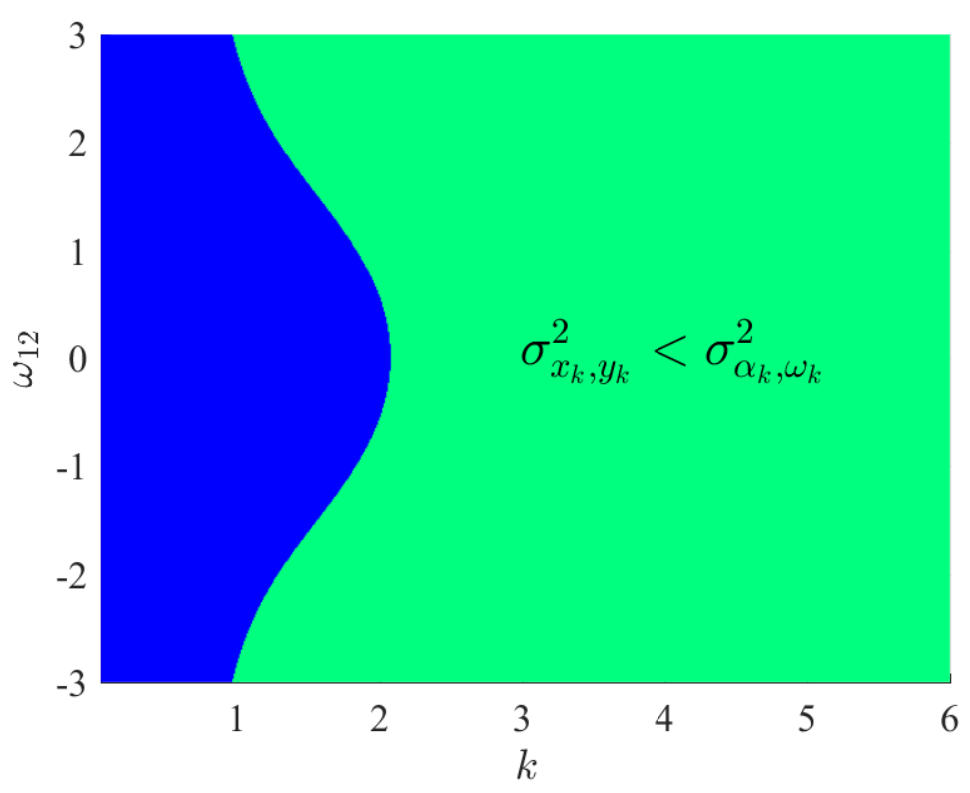

Figure 3.5: Comparison of amplitude bound (3.59) and the damping factor and frequency bound (3.60) for two damped cisoids for the MRS model. 
Section 3.5.4. For $N=1$ :

$$
d_{1}=\frac{1}{16\left(\sigma^{2} \delta\right)^{4}} \frac{a^{4}}{\alpha^{8}}
$$

For $N=2$ :

$$
\begin{aligned}
& d_{2}=\frac{1}{256\left(\sigma^{2} \delta\right)^{8}} \frac{a_{1}^{4} a_{2}^{4}}{\alpha_{1}^{8} \alpha_{2}^{8}} \zeta_{2}^{8} \\
& \zeta_{2}=\frac{\left(\alpha_{1}-\alpha_{2}\right)^{2}+\omega_{12}^{2}}{\left(\alpha_{1}+\alpha_{2}\right)^{2}+\omega_{12}{ }^{2}}
\end{aligned}
$$

For arbitrary $N$ :

$$
\begin{gathered}
d_{N}=\frac{1}{(16)^{N}\left(\sigma^{2} \delta\right)^{4 N}} \frac{a_{1}^{4} a_{2}^{4} \ldots a_{N}^{4}}{\alpha_{1}^{8} \alpha_{2}^{8} \ldots \alpha_{N}^{8}} \zeta_{N}^{8} \\
\zeta_{N}=\prod_{\substack{l, k=1 \\
l \neq k}}^{N} \frac{\left(\alpha_{k}-\alpha_{l}\right)^{2}+\omega_{k l}^{2}}{\left(\alpha_{k}+\alpha_{l}\right)^{2}+\omega_{k l}^{2}}
\end{gathered}
$$

Though $\zeta_{N}$ in (3.70) may appear similar to $\rho_{N}^{(k)}$ in (3.66), it is significantly different from $\rho_{N}^{(k)}$, as well as from $\varrho_{N}^{(k)}$ in (3.65), in the sense that it encapsulates the damping factor and frequency spacings between all cisoids rather than the damping factor and frequency spacing of a given cisoid $k$ relative to all other cisoids. The determinant of $\mathbf{J}^{-1}$, which is the inverse of (3.70), is termed the generalised variance [46]. It can be employed as an objective function for the minimisation of the uncertainty associated with parameter estimates. An optimisation procedure employing this minimisation criterion is the D-optimal design, as pointed out in Section 3.3.2.

\section{The condition number}

Using (3.46), (3.48) and (3.50) of Sections 3.6.4, 3.6.5 and 3.6.6 respectively, the following results were obtained for the condition number of the FIM associated with the MRS model. For $N=1$ :

$$
c_{1} \approx 8\left(\frac{1}{\alpha}+\frac{a^{2}}{2 \alpha^{3}}\right)\left(\alpha+\frac{2}{a^{2}} \alpha^{3}\right)
$$


For $N=2$ :

$$
c_{2} \approx 8\left\{\sum_{k=1}^{2}\left(\frac{1}{\alpha_{k}}+\frac{a_{k}^{2}}{2 \alpha_{k}^{3}}\right)\right\}\left\{\sum_{k=1}^{2}\left(\alpha_{k} \rho_{2}^{3}+\frac{2}{a_{k}^{2}} \alpha_{k}^{3} \rho_{2}^{2}\right)\right\}
$$

For arbitrary $N$ :

$$
c_{N} \approx 8\left\{\sum_{k=1}^{N}\left(\frac{1}{\alpha_{k}}+\frac{a_{k}^{2}}{2 \alpha_{k}^{3}}\right)\right\}\left\{\sum_{k=1}^{N}\left(\alpha_{k}\left\{\rho_{N}^{(k)}\right\}^{3}+\frac{2}{a_{k}^{2}} \alpha_{k}^{3}\left\{\rho_{N}^{(k)}\right\}^{2}\right)\right\}
$$

It is interesting to note that the condition number does not depend on noise variance $\sigma^{2}$ nor on the sampling rate $\delta$. Condition number can be used for the identification of unusual conditions that may lead to degeneracy of the FIM and the subsequent variations engendered in the predicted estimates, as highlighted in Section 3.3.3.

\section{Factorisation of characteristic polynomial and eigenvalue pairing}

The characteristic polynomial associated with $\mathbf{J}$ for the MRS model is of order $4 N$ due to the $4 N \times 4 N$ size of FIM, $N$ being the number of cisoids and 4 the number of parameters to be estimated for each cisoid, i.e., real and imaginary amplitude, damping factor and frequency. This $4 N$ degree polynomial can be factored into two identical $2 N$ degree polynomials.

$$
\phi(\lambda)=\Upsilon(\lambda) \Upsilon(\lambda)
$$

such that:

$$
\Upsilon(\lambda)=\left(\lambda-\lambda_{1}\right)\left(\lambda-\lambda_{2}\right) \ldots\left(\lambda-\lambda_{2 N}\right)
$$

Consequently, all the eigenvalues come in pairs and only $2 N$ of them are unique. This follows from the following pairing observed in the derivatives of parameter vector for this problem:

$$
\frac{\partial \tilde{z}(m)}{\partial y_{n}}=j \frac{\partial \tilde{z}(m)}{\partial x_{n}}
$$

And

$$
\frac{\partial \tilde{z}(m)}{\partial \omega_{n}}=-j \frac{\partial \tilde{z}(m)}{\partial \alpha_{n}}
$$


Hence, the eigenvalues of $\mathbf{J}$ for the MRS model are repeated with every eigenvalues having a multiplicity of two.

\subsubsection{The MRR model}

In MRR, the signal is modelled as a sum of $N$ real exponentials, being observed as $M$ samples, regularly spaced by $\delta$ in time:

$$
z(m)=\sum_{n=1}^{N} x_{n} e^{-\alpha_{n} m \delta}+v(m)
$$

$z(m)$ is the $m$-th observed sample of the signal vector $z$ such that $\boldsymbol{z}=$ $[z(0), z(1), \ldots, z(M-1)] . x_{n} \in \mathbb{R}$ is the amplitude and $\alpha_{n} \in \mathbb{R}$ is the decay rate of the $n$-th component with $\alpha_{n}>0$. The noise process $\boldsymbol{v}$, i.e., $\boldsymbol{v}=$ $[v(0), v(1), \ldots, v(M-1)]$, is assumed white with zero mean and variance $\sigma^{2}$. The noise-free equivalent of $z(m)$ is denoted by $\tilde{z}(m)=\sum_{n=1}^{N} x_{n} e^{-\alpha_{n} m \delta}$.

\section{The FIM}

The MRR model (3.79) can be considered a special case of the MRS model (3.51), with the complex part of the amplitude and frequency of each cisoid in (3.51) set to zero. The parameter vector $\boldsymbol{\theta}$ for the MRR model is reduced from that in (3.52) to:

$$
\boldsymbol{\theta}=\left[\begin{array}{lllll}
x_{1} & \alpha_{1} & x_{2} & \ldots & \alpha_{N}
\end{array}\right]^{T}
$$

The $i j$-th element of the FIM for real data $z$, parameterised by real vector $\boldsymbol{\theta}$ independent of noise variance $\sigma^{2}$, is expressed as:

$$
\mathbf{J}=\frac{1}{\sigma^{2}}\left[\begin{array}{cccc}
\mathbf{T}^{11} & \mathbf{T}^{12} & \ldots & \mathbf{T}^{1 N} \\
\mathbf{T}^{21} & \mathbf{T}^{22} & \ldots & \mathbf{T}^{2 N} \\
\vdots & \vdots & \ddots & \vdots \\
\mathbf{T}^{N 1} & \mathbf{T}^{N 2} & \ldots & \mathbf{T}^{N N}
\end{array}\right]
$$


The dimensions of $\mathbf{J}$ are $2 N \times 2 N$. $\mathbf{T}^{k l}$ are $2 \times 2$ block matrices the elements of which correspond to the $k$-th and $l$-th exponentials [232], i.e., $T_{11}^{k l}=$ $\sum_{m=0}^{M-1} \frac{\partial \tilde{z}(m)^{T}}{\partial x_{k}} \frac{\partial \tilde{z}(m)}{\partial x_{l}}$.

\section{Properties of the FIM and the signal model}

The FIM and signal properties for the MRS model discussed in Sections 3.7.1 and 3.7.1 respectively also hold for the MRR model. Specifically, the substitutions proposed in (3.55) and (3.56) and the subsequent relationships expressed in Table 3.1 (between the elements of the FIM) can also employed in computation of (3.81) after setting $p_{n}=e^{-\alpha_{n} \delta}$. The CRLB is computed by inverting $\mathrm{J}$.

\section{The CRLB}

The following bounds are obtained from the diagonal elements of $\mathbf{J}^{-1}$ using (3.9), (3.10) and (3.11) of Section 3.5.2 and (3.15) of Section 3.5.3. For $N=1$ :

$$
\sigma_{x}^{2} \geq \delta \sigma^{2} 4 \alpha
$$

and

$$
\sigma_{\alpha}^{2} \geq \frac{\delta \sigma^{2}}{a^{2}} 8 \alpha^{3}
$$

For $N=2$ :

$$
\sigma_{x_{k}}^{2} \geq \delta \sigma^{2} 4 \alpha_{k} \varrho_{2}
$$

and

$$
\sigma_{\alpha_{k}}^{2} \geq \frac{\delta \sigma^{2}}{x_{k}^{2}} 8 \alpha_{k}^{3} \rho_{2}^{2}
$$

with

$$
\varrho_{2}=\frac{\left(\alpha_{1}+\alpha_{2}\right)^{2}\left\{\alpha_{1}^{4}-8 \alpha_{1}^{3} \alpha_{2}+30 \alpha_{1}^{2} \alpha_{2}^{2}+8 \alpha_{1} \alpha_{2}^{3}+\alpha_{2}^{4}\right\}}{\left(\alpha_{1}-\alpha_{2}\right)^{6}}
$$

and

$$
\rho_{2}=\frac{\left(\alpha_{1}+\alpha_{2}\right)^{2}}{\left(\alpha_{1}-\alpha_{2}\right)^{2}}
$$


For arbitrary $N$ :

$$
\sigma_{x_{k}}^{2} \geq \delta \sigma^{2} 4 \alpha_{k} \varrho_{N}^{(k)}
$$

and

$$
\sigma_{\alpha_{k}}^{2} \geq \frac{\delta \sigma^{2}}{x_{k}^{2}} 8 \alpha_{k}^{N}\left\{\rho_{3}^{(k)}\right\}^{2}
$$

with

$$
\varrho_{N}^{(k)}=\prod_{\substack{l=1 \\ l \neq k}}^{N} \frac{\left(\alpha_{k}+\alpha_{l}\right)^{2}\left\{\alpha_{k}^{4}-8 \alpha_{k}^{3} \alpha_{l}+30 \alpha_{k}^{2} \alpha_{l}^{2}+8 \alpha_{k} \alpha_{l}^{3}+\alpha_{l}^{4}\right\}}{\left(\alpha_{k}-\alpha_{l}\right)^{6}}
$$

and

$$
\rho_{N}^{(k)}=\prod_{\substack{l=1 \\ l \neq k}}^{N} \frac{\left(\alpha_{k}+\alpha_{l}\right)^{2}}{\left(\alpha_{k}-\alpha_{l}\right)^{2}}
$$

The interaction terms (3.86) and (3.87) for bound results (3.84) and (3.85) are displayed in Figures 3.6 and 3.7 respectively. It can be observed that the uncertainty associated with the parameter estimation of the MRR model is relatively large when compared with that of the MRS model. This is further highlighted in the overlaid plot of (3.86) and (3.87) as a function of $k\left(\alpha_{1}=k \alpha_{2}\right)$ in Figure 3.8. In order to ensure a meaningful comparison between the two, the results displayed for (3.87) in Figure 3.8 are raised to a power of two, i.e., $\rho_{2}^{2}$, this being its magnitude appearing in (3.85). Figure 3.8 also shows that the magnitude of (3.87) is greater than that of (3.86) for $k>5$. This implies that the uncertainty associated with the estimation of damping factor of a real exponential can be higher than that associated with its amplitude. To further elucidate this point, a point-by-point comparison of (3.84) and (3.85) as a function of $\alpha_{1}$ and $\alpha_{2}$ is displayed in Figure 3.9. The comparison reveals a similar observation as was made for the MRS model in Section 3.7.1, i.e., in certain cases the uncertainty associated with estimation of damping factor can exceed the uncertainty associated with the estimation of the amplitude. Jointly, these observations lead to the following general remark. 


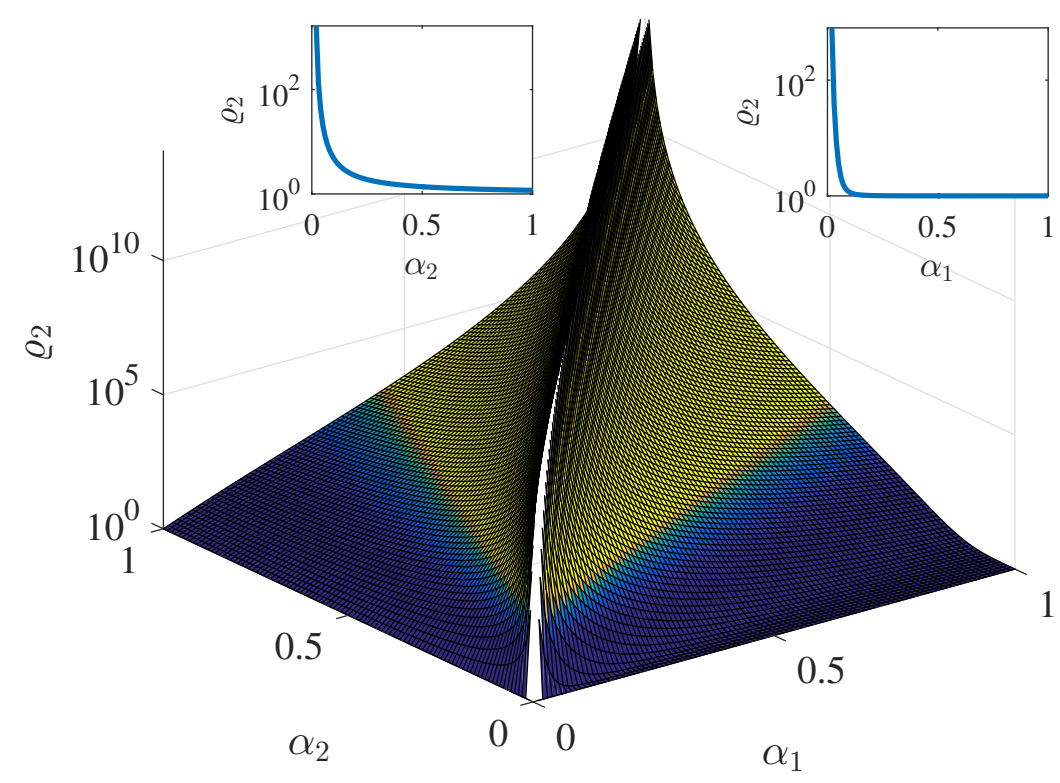

Figure 3.6: Amplitude interaction term (3.86) as a function of $\alpha_{1}$ and $\alpha_{2}$ for the MRR model.

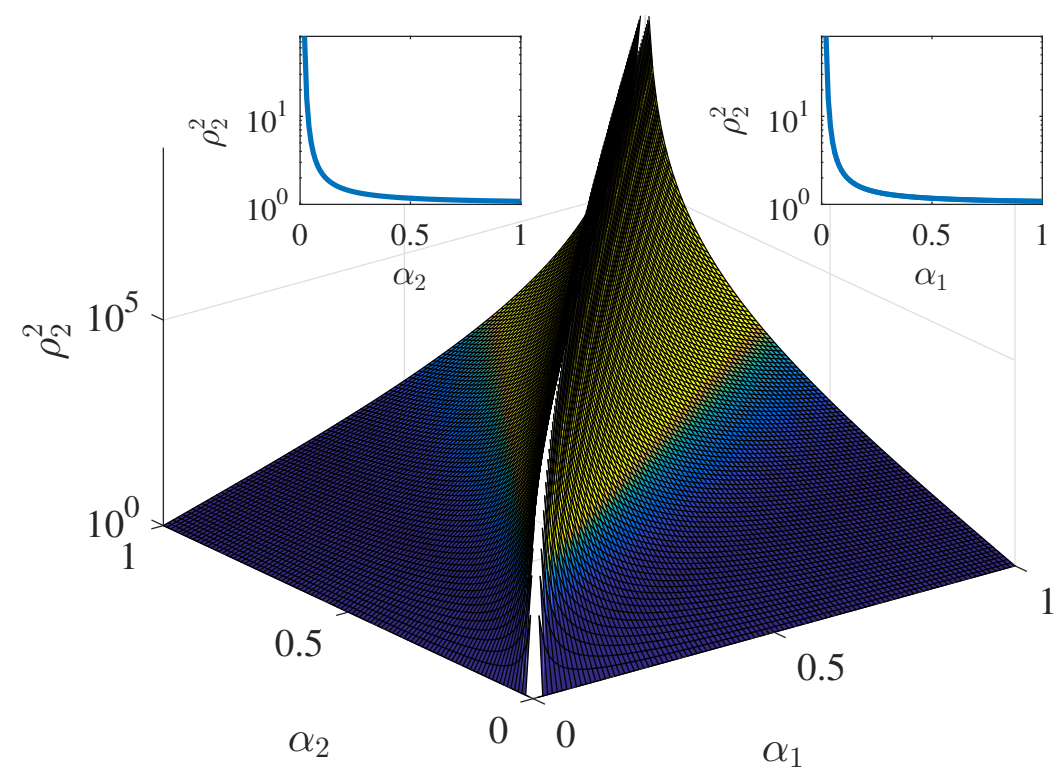

Figure 3.7: Damping factor and frequency interaction term (3.87) as a function of $\alpha_{1}$ and $\alpha_{2}$ for the MRR model. 


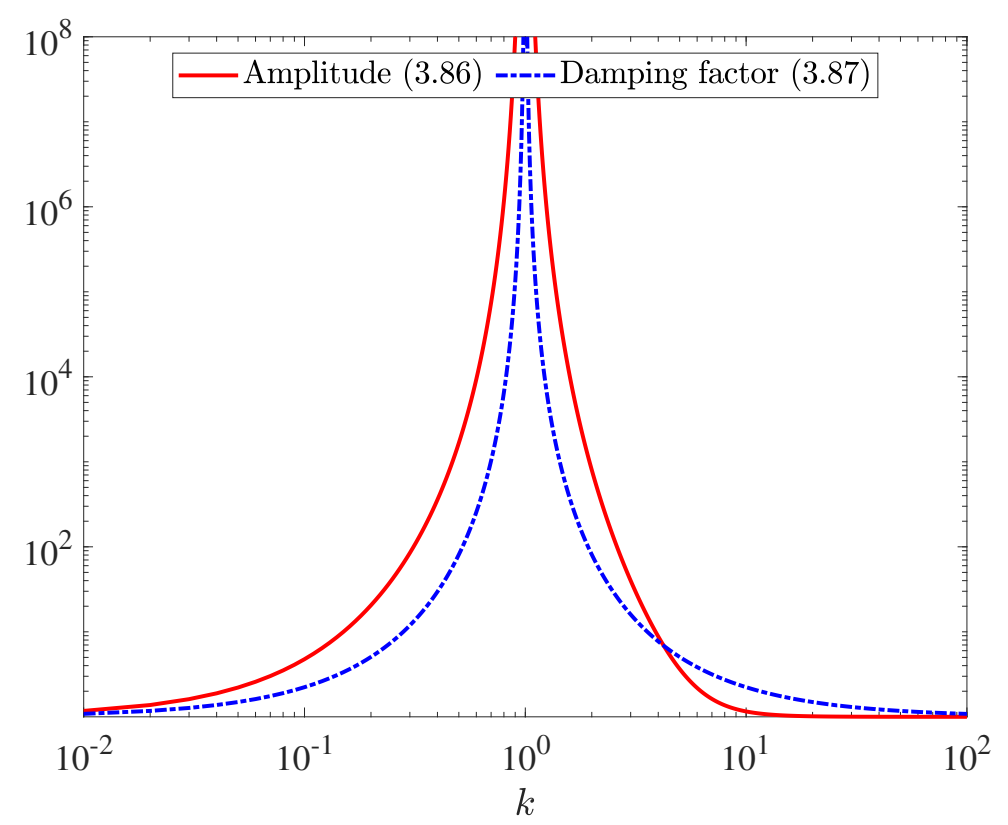

Figure 3.8: Logarithmic plot of amplitude and damping factor and frequency interaction terms (3.86) and (3.87) as a function of $k$ with $\alpha_{1}=k \alpha_{2}$ for the MRR model.

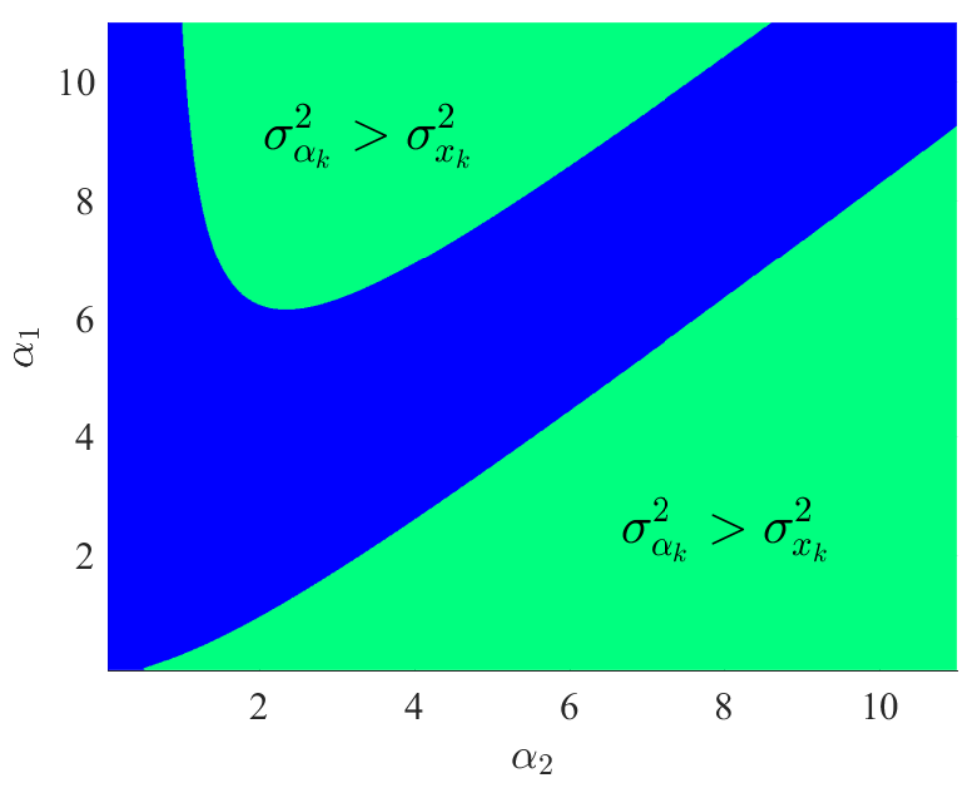

Figure 3.9: Comparison of amplitude bound (3.84) and the damping factor bound (3.85) for two exponentials for the MRR model. 
A general remark on the comparative relationship between parameter bounds The bound on the damping factor and/or frequency of a cisoid is not always lower than the bound on its real and/or imaginary parts of the amplitude, and in certain cases, may exceed the latter. This also holds for the case of real exponentials.

\section{The determinant}

Equations (3.46), (3.48) and (3.50) of Sections 3.6.4, 3.6.5 and 3.6.6 respectively were employed to compute the determinant of FIM associated with the MRR model. For $N=1$ :

$$
d_{1}=\frac{1}{16\left(\sigma^{2} \delta\right)^{2}} \frac{x^{2}}{\alpha^{4}}
$$

For $N=2$ :

$$
\begin{gathered}
d_{2}=\frac{1}{256\left(\sigma^{2} \delta\right)^{4}} \frac{x_{1}^{2} x_{2}^{2}}{\alpha_{1}^{4} \alpha_{2}^{4}} \zeta_{2}^{4} \\
\zeta_{2}=\frac{\left(\alpha_{1}-\alpha_{2}\right)}{\left(\alpha_{1}+\alpha_{2}\right)}
\end{gathered}
$$

For arbitrary $N$ :

$$
\begin{gathered}
d_{N}=\frac{1}{(16)^{N}\left(\sigma^{2} \delta\right)^{2 N}} \frac{x_{1}^{2} x_{2}^{2} \ldots x_{N}^{2}}{\alpha_{1}^{4} \alpha_{2}^{4} \ldots \alpha_{N}^{4}} \zeta_{N}^{4} \\
\zeta_{N}=\prod_{\substack{l, k=1 \\
l \neq k}}^{N} \frac{\left(\alpha_{k}-\alpha_{l}\right)}{\left(\alpha_{k}+\alpha_{l}\right)}
\end{gathered}
$$

\section{No pairing of eigenvalues}

We saw earlier in Section 3.7.1 that the $4 N$ eigenvalues for the MRS model are observed in pairs, and only $2 N$ of them are unique. This pairing arose from the relationship between real and imaginary components of the amplitude and the damping factor and frequency derivatives of parameter vector for this problem, and was depicted in (3.77) and (3.78) respectively. As the MRR model constitutes only real exponentials with real 
amplitudes, the parameter vector for this model does not contain the terms for imaginary amplitude and frequency. The derivatives of the parameter vector with respect to these parameters are not taken and the relation observed no longer exists. Consequently, the eigenvalue pairing is dropped and $2 N$ unique eigenvalues are observed for the MRR model.

\section{The condition number}

Equations (3.46), (3.48) and (3.50) of Sections 3.6.4, 3.6.5 and 3.6.6 respectively were employed to compute the condition number of the FIM for the MRR model. For $N=1$ :

$$
c_{1} \approx 2\left(\frac{1}{\alpha}+\frac{x^{2}}{2 \alpha^{3}}\right)\left(\alpha+\frac{2}{x^{2}} \alpha^{3}\right)
$$

For $N=2$ :

$$
c_{2} \approx 2\left\{\sum_{k=1}^{2}\left(\frac{1}{\alpha_{k}}+\frac{x_{k}^{2}}{2 \alpha_{k}^{3}}\right)\right\}\left\{\sum_{k=1}^{2}\left(\alpha_{k} \rho_{2}^{3}+\frac{2}{x_{k}^{2}} \alpha_{k}^{3} \rho_{2}^{2}\right)\right\}
$$

For arbitrary $N$ :

$$
c_{N} \approx 2\left\{\sum_{k=1}^{N}\left(\frac{1}{\alpha_{k}}+\frac{x_{k}^{2}}{2 \alpha_{k}^{3}}\right)\right\}\left\{\sum_{k=1}^{N}\left(\alpha_{k}\left\{\rho_{N}^{(k)}\right\}^{3}+\frac{2}{x_{k}^{2}} \alpha_{k}^{3}\left\{\rho_{N}^{(k)}\right\}^{2}\right)\right\}
$$

\subsubsection{The MRS model with previous knowledge}

We now investigate a special case of the MRS model which enables the incorporation of prior knowledge into the model for estimation purposes, specifically the case in which the frequencies and damping factors are known and only the real and imaginary part of the amplitude need to be determined. This case, as we saw in Section 3.2.3, frequently arises in complex mixture analysis in which only the concentrations of chemicals need to be ascertained [229]. Henceforth, this model shall be referred to as 
the amplitude-only model. For this problem, the parameter vector of the MRS model in (3.52) reduces to:

$$
\boldsymbol{\theta}=\left[\begin{array}{lllllll}
x_{1} & y_{1} & x_{2} & y_{2} & \ldots & x_{N} & y_{N}
\end{array}\right]^{T}
$$

Consequently, the block matrices $\mathrm{T}^{k l}$ inside the FIM are now $2 \times 2$ sized blocks, and the size of FIM is $2 N \times 2 N$. All other relationships for $\mathbf{J}$, mentioned in Sections 3.7.1 and 3.7.1, remain intact.

\section{The CRLB}

Using (3.9), (3.10), and (3.11) of Section 3.5.2 and (3.15) of Section 3.5.3, the following CRLB expressions were obtained for the amplitude-only model from the diagonal elements of $\mathbf{J}^{-1}$. For $N=1$ :

$$
\sigma_{x}^{2}=\sigma_{y}^{2} \geq \delta \sigma^{2} \alpha
$$

For $N=2$ :

$$
\sigma_{x_{k}}^{2}=\sigma_{y_{k}}^{2} \geq \delta \sigma^{2} \alpha_{k} \rho_{2}
$$

with

$$
\rho_{2}=\frac{\left(\alpha_{1}+\alpha_{2}\right)^{2}+\omega_{12}^{2}}{\left(\alpha_{1}-\alpha_{2}\right)^{2}+\omega_{12}^{2}}
$$

For arbitrary $N$ :

$$
\sigma_{x_{k}}^{2}=\sigma_{y_{k}}^{2} \geq \delta \sigma^{2} \alpha_{k} \rho_{N}^{(k)}
$$

with

$$
\rho_{N}^{(k)}=\prod_{\substack{l=1 \\ l \neq k}}^{N} \frac{\left(\alpha_{k}+\alpha_{l}\right)^{2}+\omega_{k l}^{2}}{\left(\alpha_{k}-\alpha_{l}\right)^{2}+\omega_{k l}^{2}}
$$

An interesting observation that immediately follows from (3.102) is that the interaction term for the amplitude bound of the amplitude-only model, though a special case of the MRS model, does not follow the corresponding interaction term of the latter, but is identical to the interaction term for the damping factor and frequency bounds in (3.60). Furthermore, unlike 
that in (3.60), the interaction term in (3.102) is not raised to the power of two. This signifies a marked reduction in the uncertainty in the estimation of the amplitudes for the amplitude-only model, signifying the advantage of prior knowledge. This advantage is displayed in Figure 3.10 where the interaction term (3.103) for $N=2$ is plotted as a function of $\omega_{12}$ and $k$ with $\alpha_{1}=\alpha_{2}$. However, to view this more closely, a further comparison showing an overlaid comparison of this interaction term with the amplitude and damping factor and frequency interaction terms for the MRS model as a function of $\omega_{12}$ and $k$ are displayed in Figures 3.11 and 3.12 respectively. The comparison presented in Figures 3.11 and 3.12 confirms the marked advantage of the inclusion of previous knowledge in the MRS model.

\section{The determinant}

The following results were obtained for the determinant of the FIM associated with the amplitude-only model using (3.46), (3.48) and (3.50) of Sections 3.6.4, 3.6.5 and 3.6.6 respectively. For $N=1$ :

$$
d_{1}=\left(\delta \sigma^{2}\right)^{2} \alpha^{2}
$$

For $N=2$ :

$$
d_{2}=\frac{\left(\delta \sigma^{2}\right)^{4}}{\alpha_{1}^{2} \alpha_{2}^{2}} \zeta_{2}^{2}
$$

with

$$
\zeta_{2}=\frac{\left(\alpha_{1}-\alpha_{2}\right)^{2}+\omega_{12}^{2}}{\left(\alpha_{1}+\alpha_{2}\right)^{2}+\omega_{12}^{2}}
$$

For arbitrary $N$ :

$$
d_{N}=\frac{\left(\delta \sigma^{2}\right)^{2 N}}{\alpha_{1}^{2} \alpha_{2}^{2} \ldots \alpha_{N}^{2}} \zeta_{N}^{2}
$$

with

$$
\zeta_{N}=\prod_{\substack{l, k=1 \\ l \neq k}}^{N} \frac{\left(\alpha_{k}-\alpha_{l}\right)^{2}+\omega_{k l}^{2}}{\left(\alpha_{k}+\alpha_{l}\right)^{2}+\omega_{k l}^{2}}
$$




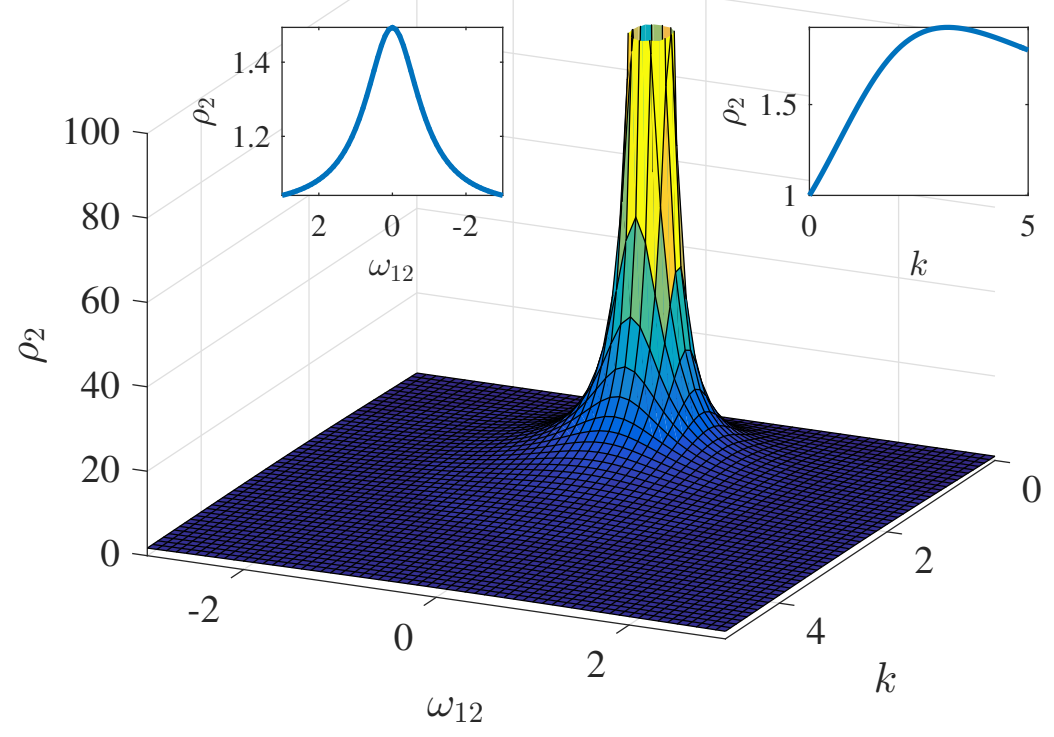

Figure 3.10: Amplitude interaction term (3.103) as a function of $\omega_{12}$ and $k$ $\left(\alpha_{1}=k \alpha_{2}\right)$ for the amplitude-only model.

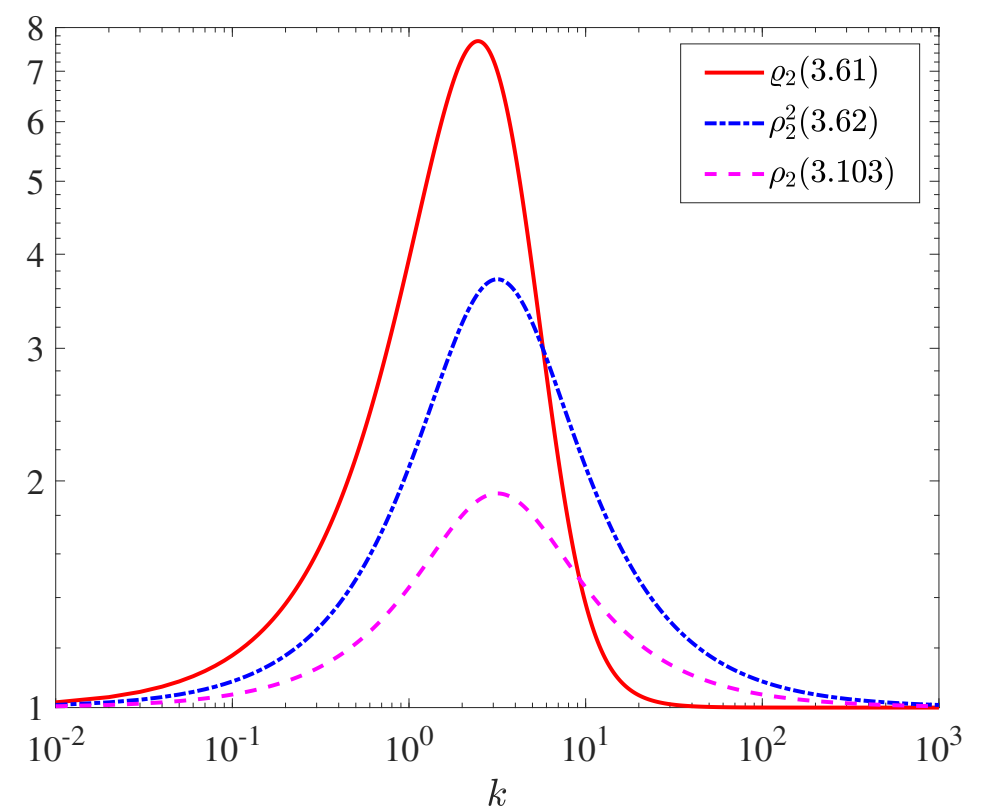

Figure 3.11: Logarithmic plot of the amplitude interaction term (3.103) of the amplitude-only model and the amplitude and damping factor and frequency interaction terms (3.61) and (3.62) of the MRS model as a function of $k$ with $\alpha_{1}=k \alpha_{2}$. 


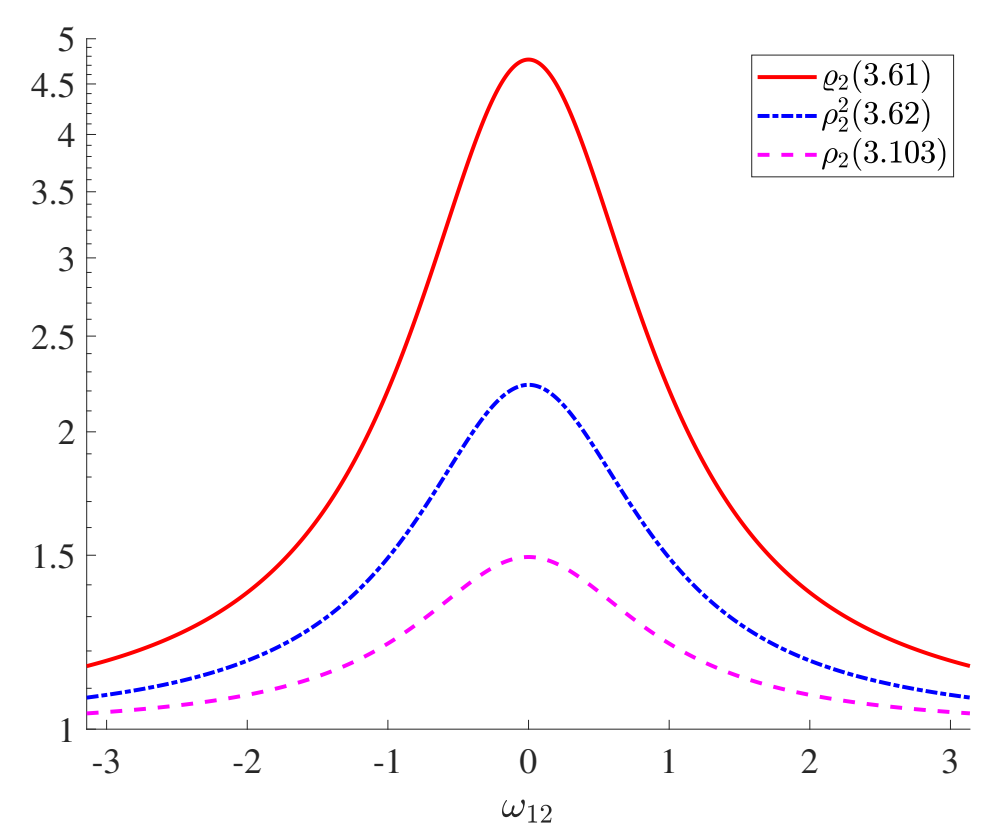

Figure 3.12: Logarithmic magnitude plot of the amplitude interaction term (3.103) of the amplitude-only model and the amplitude and damping factor and frequency interaction terms (3.61) and (3.62) of the MRS model as a function of $\omega_{12}$.

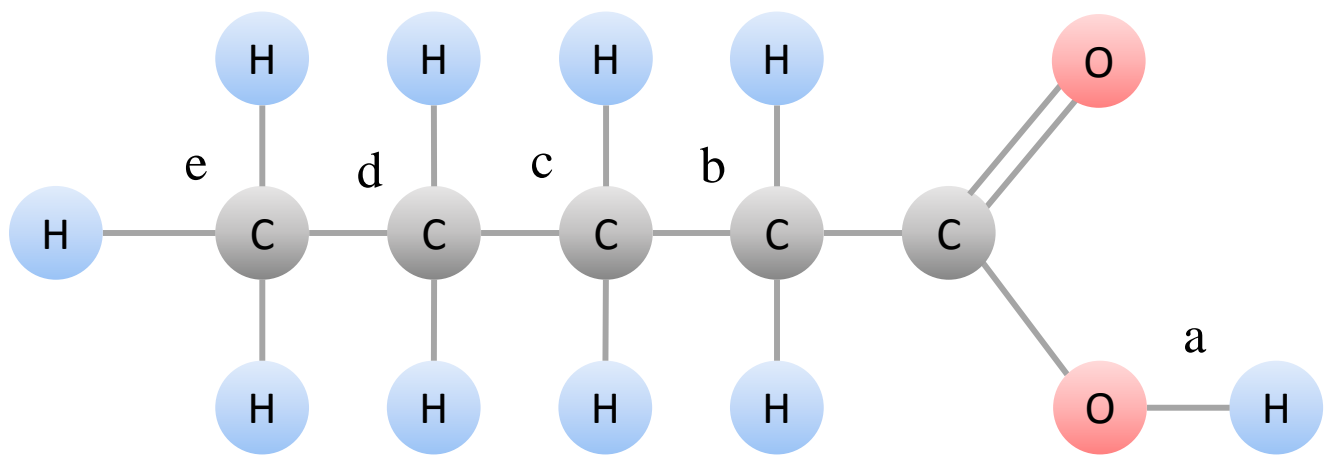

Figure 3.13: Chemical structure of pentanoic acid [12]. 


\section{Factorisation of determinant eigenvalue pairing}

Just as we observed for the MRS model in Section 3.7.1, the eigenvalues for the amplitude-only model are also paired, i.e., there are $N$ unique eigenvalues with every eigenvalues having a multiplicity of two. This - as discussed in Section 3.7.1 - can be attributed to the following relationship between the derivatives of $\hat{z}(m)$ in (3.51) when taken with respect to the elements of the parameter vector $\boldsymbol{\theta}$ for the amplitude-only model expressed in (3.100):

$$
\frac{\partial \tilde{z}(m)}{\partial y_{n}}=j \frac{\partial \tilde{z}(m)}{\partial x_{n}}
$$

Consequently, the determinant can also be factored into two identical parts:

$$
d_{N}=\left\{\frac{\left(\delta \sigma^{2}\right)^{N}}{\alpha_{1} \alpha_{2} \ldots \alpha_{N}} \zeta_{N}\right\}\left\{\frac{\left(\delta \sigma^{2}\right)^{N}}{\alpha_{1} \alpha_{2} \ldots \alpha_{N}} \zeta_{N}\right\}
$$

with

$$
\zeta_{N}=\prod_{\substack{l, k=1 \\ l \neq k}}^{N} \frac{\left(\alpha_{k}-\alpha_{l}\right)^{2}+\omega_{k l}^{2}}{\left(\alpha_{k}+\alpha_{l}\right)^{2}+\omega_{k l}^{2}}
$$

This observation about the eigenvalue paring and its relationship to the derivatives of the parameter vector, and a similar observation made in Section 3.7.1 for the MRS model, lead to the following general remark.

A general remark on the pairing of eigenvalues of the Fisher Information Matrix A pairing is observed in the eigenvalues of the Fisher Information Matrix associated with a given signal model if its derivatives with respect to the parameter vector to be estimated are also paired (by a multiplicative factor which may be complex).

\section{The condition number}

The expressions obtained for the condition number of the FIM associated with the amplitude-only model, computed using (3.46), (3.48) and (3.50) 
of Sections 3.6.4, 3.6.5 and 3.6.6 respectively, are presented as follows. For $N=1$ :

$$
c_{1}=1
$$

For $N=2$ :

$$
c_{2} \approx 4\left\{\sum_{k=1}^{2} \frac{1}{\alpha_{k}}\right\}\left\{\sum_{k=1}^{2} \alpha_{k} \rho_{2}\right\}
$$

For arbitrary $N$ :

$$
c_{N} \approx 4\left\{\sum_{k=1}^{N} \frac{1}{\alpha_{k}}\right\}\left\{\sum_{k=1}^{N} \alpha_{k} \rho_{N}^{(k)}\right\}
$$

\subsection{Application}

The theoretical results presented in Section 3.7 are now illustrated by their application to ${ }^{1} \mathrm{H}$ MRS data of pentanoic acid (also known as valeric acid). Pentanoic acid is an alkyl carboxylic acid with the chemical formula $\mathrm{C}_{5} \mathrm{H}_{10} \mathrm{O}_{2}$. Its ${ }^{1} \mathrm{H}$ NMR spectrum can be approximately characterised by the " $n+1$ rule" [12]. This rule predicts the multiplicity of spectral peaks in the pentanoic acid spectrum that arise due to the spin-spin coupling between its hydrogen atoms. The chemical structure of pentanoic acid is displayed in Figure 3.13. The pentanoic acid molecule contains: one carboxyl $\mathrm{CO}_{2} \mathrm{H}$ group (a); three methylene $\mathrm{CH}_{2}$ groups (b), (c) and (d); and one methyl $\mathrm{CH}_{3}$ group (e). They will now be analysed in the same order according to the " $\mathrm{n}+1$ rule".

The hydrogen atom in the carboxyl group (a) generates the highest spectral peak which, due to the lack of immediately neighbouring hydrogen atoms, is unsplit. This peak is highest in the spectrum due to an electronegative oxygen atom present in the group. The $\mathrm{CH}_{2}$ group (b) lies closest to this oxygen atom. It is split into a triplet due to the two hydrogen atoms present in the neighbouring $\mathrm{CH}_{2}$ group (c), i.e., $(2+1)=3$; the triplet (b) is observed alongside the large water peak. Next in line is the $\mathrm{CH}_{2}$ group (c) which is split into a pentuplet (five peaks) due to four 


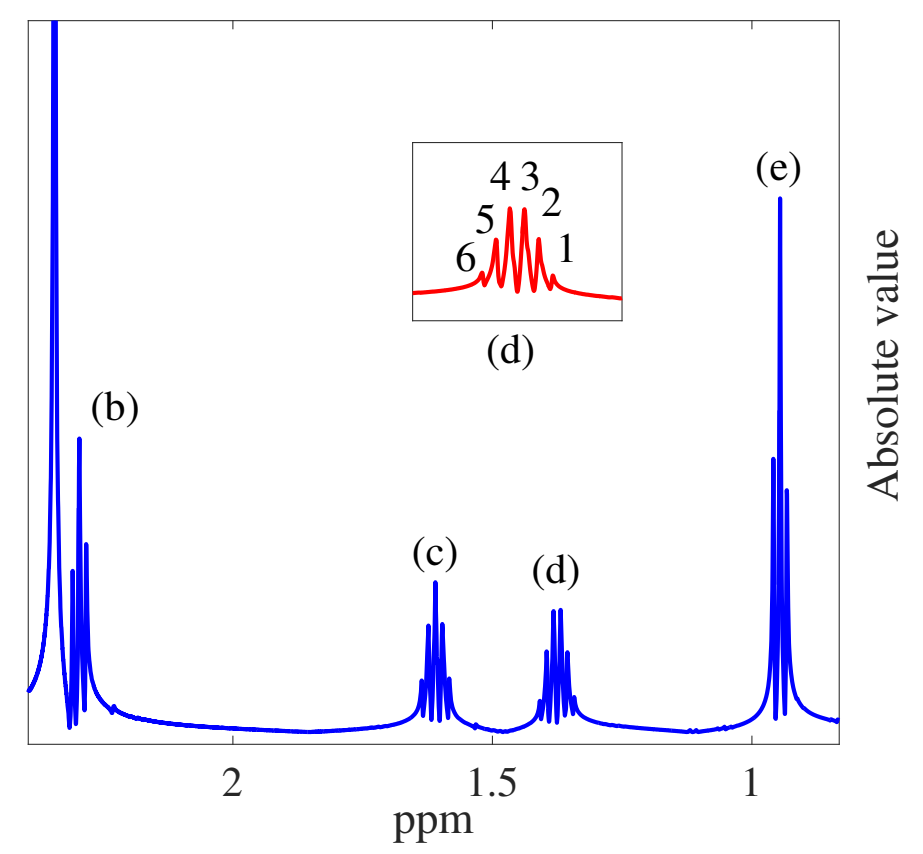

Figure 3.14: ${ }^{1} \mathrm{H}$ NMR spectrum of pentanoic acid.

neighbouring hydrogen atoms, i.e., two in (b) and two in (d). The $\mathrm{CH}_{2}$ group (d) is split into a sextuplet(six peaks) due to two hydrogen atoms in (c) and three in (e). Finally, the $\mathrm{CH}_{3}$ group (e) is split into a triplet by two hydrogen atoms in (d). These groups as well as their locations are depicted in Figure 3.14. Individual labelling scheme for peaks inside a multiplet is displayed in the inset of Figure 3.14, and shall likewise be followed for all multiplets in Table 3.2 and onwards.

It is apparent from the above that a relatively simple compound of pentanoic acid with first-order spectra can generate multiplets with up to six peaks. The resolution of these multiplets is important for the determination of the fine chemical structure of the compound. The ability of a quantitation method to resolve such multiples, i.e., how accurately such peaks can be estimated under given experimental conditions, can be analytically examined by the CRLB. But this requires analytical CRLB expressions ap- 
plicable to multiplets of higher order than has previously been available.

To demonstrate the efficacy of the derived results in this regard, we apply them to the ${ }^{1} \mathrm{H}$ NMR spectrum of the pentanoic acid depicted in Figure 3.14. The Free Induction Decay (FID) signal of the pentanoic acid was recorded on a $60 \mathrm{MHz}$ spectrometer (Spinsolve Benchtop NMR; Magritek, Wellington, New Zealand). The FID quantitation was performed using the subband Steiglitz-McBride algorithm proposed in Chapter 5 for the quantitation of 1D-NMR signals. The algorithm converts the FID signal into a table of amplitudes, phases, frequencies and damping factors. The quantitation results thus obtained are employed to compute the interaction terms for the MRS, MRR and the amplitude-only model using (3.65), (3.66), (3.90), (3.91) and (3.105) respectively. The interaction terms are specifically considered because they essentially encapsulate the behaviour of the CRLB with respect to the model under consideration and the multiplicity of the peaks involved.

Calculations for the interaction terms are carried out in two steps. In the first step, the calculations are made for individual multiplet groups, independent of each other, and are displayed in Table $3.2^{1}$. This is performed explicitly to observe the influence of model type, prior knowledge, asymmetry or any other imperfection that may be present in the individual multiplet groups. In the second step, incremental calculations - when an extraneous peak is observed in the neighbourhood of a multiplet - are made for the interaction term for a given multiplet in order to analyse the impact of extraneous peaks on the accuracy of estimation. Specifically, these calculations were performed to analyse the impact of solvent peaks and carbon satellites, discussed in Sections 3.8.4 and 3.8.5 and displayed in Tables 3.3 and 3.4 respectively.

\footnotetext{
${ }^{1}$ The results depicted for the MRR model are synthetic and do not correspond to any real experiment.
} 


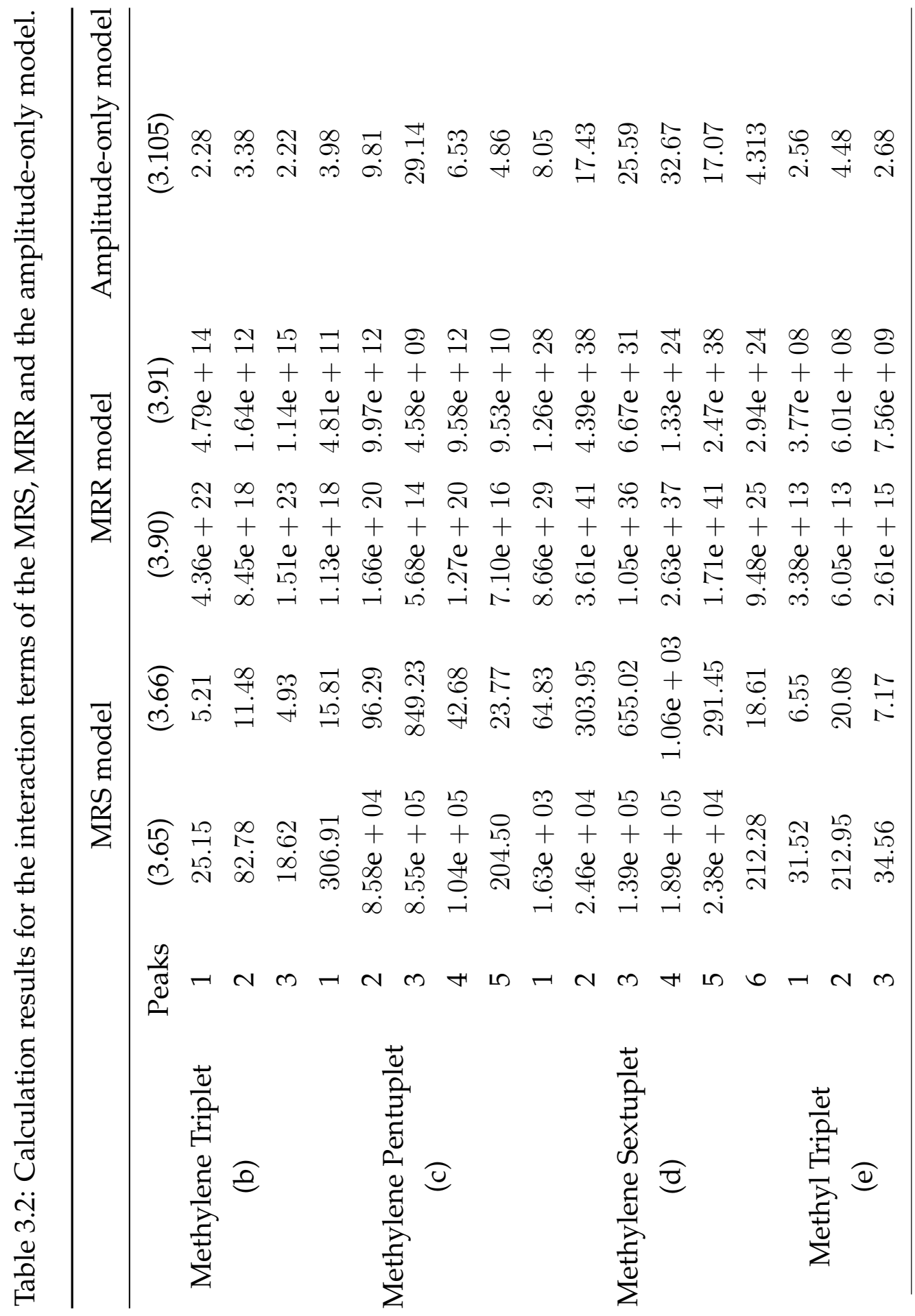




\subsubsection{Impact of model type}

It is evident from Table 3.2 that, for all the results displayed, the interaction terms with highest magnitude are associated with the MRR model and those with the lowest amplitude are associated with the amplitudeonly model. Even for a triplet, the magnitude of the interaction terms for the MRR model is almost $10^{11}$ times higher than those of the MRS model, which is due to the lack of frequency information in the former. The MRR model has been shown to be highly ill-conditioned [86]. Several methods have been proposed in the literature for analysis of MRR data. However, due to the inherent complexity associated with this problem as shown above, these methods suffer from limited resolution, low detection power, and large statistical variance [198].

On the other hand, the relatively small magnitude of the interaction terms in the amplitude-only model displays the advantage of having prior knowledge. This can be explained by considering the relationship between the interaction terms derived for the MRS model and the amplitudeonly model. The interaction term for the real and imaginary part of the amplitude of the latter (3.105) is equal to the square-root of the interaction term for the damping factor and frequency of the former (3.66). Due to the square-root - and due to the fact that the magnitude of (3.66) is much smaller than that of its amplitude counterpart (3.65) in the vicinity of damping factor and frequency singularities (demonstrated in Figures 3.3 and 3.4) - a much smaller magnitude of (3.105) is observed for the amplitude-only model in all cases. This advantage has been widely exploited for parameter estimation in a broad range of MRS problems $[48,196,222]$.

\subsubsection{Impact of multiplicity}

As observed from Table 3.2, the magnitude of the interaction terms for all model types increases with increase in the multiplicity. This increase in 
the magnitude of the interaction terms increases the uncertainty associated with each cisoid in turn. This is because the interaction terms encapsulate the relative impact of the peaks present in the spectrum on the peak under consideration. On the other hand, the local terms, discussed explicitly in Section 3.7.1, depend only on the local properties of the peak whose parameter are being estimated, and not on its interaction with the rest of the peaks in the spectrum.

\subsubsection{Impact of symmetry}

Considering the damping factor and frequency interaction term (3.66) for the MRS model first, we observe that in a multiplet with an odd number of peaks, say in triplet (e), the largest value of (3.62), and hence the largest damping factor and frequency estimation uncertainty, is associated with the centre peak. This can be explained by observing that the magnitude of (3.62) for a given peak is inversely proportional to the frequency spacing of that peak relative to all other peaks. In this regard, the centre peak, being equidistant from the neighbouring peak, is more closely influenced and has the the highest value of (3.66). This also suggests that the neighbouring peaks, both being at same frequency distance from the centre peak and each other, should have identical values of (3.66) and hence, identical uncertainty associated with the estimation of their damping factors and frequencies. The value of (3.66) for these peaks, though close, is not identical.

This asymmetry becomes evident for the amplitude interaction term (3.65) for the MRS model, and is pronounced with increase in the order of multiplets, i.e., in pentuplet and sextuplet (d). This can be attributed to the nature of (3.65) which, when compared with (3.66), depicts a much more complicated interaction of the given peak with other peaks in the spectrum. This was pointed out in Section 3.7.1: that even for two cisoids, dependence of (3.65) on the individual cisoid damping factors is not straight- 
forward, i.e., see (3.61). This makes (3.65) highly asymmetric with respect to $k\left(\alpha_{1}=k \alpha_{2}\right)$ and consequently it exhibits much heavier tails for $k>5$ than displayed by (3.66). This was shown in Figure 3.3 for the theoretical results obtained from (3.62) and (3.61).

Yet another interesting observation concerns the interaction term of the amplitude-only model expressed in (3.105). This term, rather than following the amplitude interaction term of the MRS model (from which the model follows after the incorporation of prior knowledge about damping factors and frequencies) follows the behaviour of its damping factor and frequency interaction term (3.66).

Furthermore, no symmetry is observed in interaction terms for the MRR model, even for first-order splitting, i.e., the triplets. This is because the MRR model consists of real exponentials only. The interaction terms for this model, (3.90) and (3.91), exclusively depend on the damping factor spacing of a given exponential relative to all other exponentials. The frequency spacing information, encapsulating the symmetry arising due to the multiplicity of spectral peaks from the spin-spin splitting, is not employed by (3.90) and (3.91). Splitting does, however, serve to increase the number of components in (3.79) which populates (3.90) and (3.91) and drastically increases their magnitude in Table 3.2. This is in line with our earlier theoretical analysis of (3.90) and (3.91) carried in Section 3.7.2, as well as results displayed for (3.86) and (3.87) in Figure 3.8.

\subsubsection{Impact of solvent peak}

Solvents are required for sample preparation in NMR experiments. This invariably results in a large solvent (e.g., water) peak in the neighbourhood of the peaks of interest. By peaks of interest, we mean the peaks that arise due to the molecular structure of the chemical under investigation. The solvent peak is usually considered a nuisance as it serves to obscure the peaks of interest and makes estimation of their parameters dif- 
ficult [176]. Many methods, hardware and software-based, have been proposed to eliminate these peaks from the generated spectrum [9, 104, 135]. In Figure 3.14, we see the presence of a large water peak in the vicinity of the triplet (b). The calculations displayed for triplet (b) in Table 3.2 were made without considering this peak. In Table 3.3, calculations made for the MRS model inclusive of the water peak, alongside those without, are displayed for comparison. Inclusion of the water peak exhibits an increase in the magnitude of interaction terms. This, in turn, implies an increase in the estimation uncertainty associated with parameters of triplet (b) peaks. However, we observe that, despite the relatively large magnitude of the water peak compared with those of triplet peaks, increase in the magnitude of triplet (b) interaction terms is not proportionally large. In order to probe this observation further, we perform similar calculations for triplet (e) for another type of extraneous peaks, the carbon satellites, which can also occur in the neighbourhood of the peaks of interest, but are relatively small in magnitude.

\subsubsection{Impact of carbon satellites}

Carbon satellites are small peaks appearing in the neighbourhood of large peaks in ${ }^{1} \mathrm{H}$ NMR spectra [195]. They arise due to the spin-spin coupling of hydrogen atoms to neighbouring carbon 13 atoms, i.e., as a result of ${ }^{13} \mathrm{C}-{ }^{1} \mathrm{H}$ coupling. Two important properties of carbon satellites which distinguish them are: (a) they appear as evenly spaced peaks on either side of the ${ }^{1} \mathrm{H}$ peak, and (b) their order of splitting is the same as that of the ${ }^{1} \mathrm{H}$ peak they surround [195]. These properties we immediately observe in the small peaks surrounding the triplet (e) of the pentanoic acid molecule in Figure 3.15 (further pointed out by arrows): the satellites appear on either side of the triplet, and their order of multiplicity (on both sides) is the same as that of the triplet, i.e., 3. Calculations of the interaction terms for triplet (e), including the carbon satellites, are presented in 


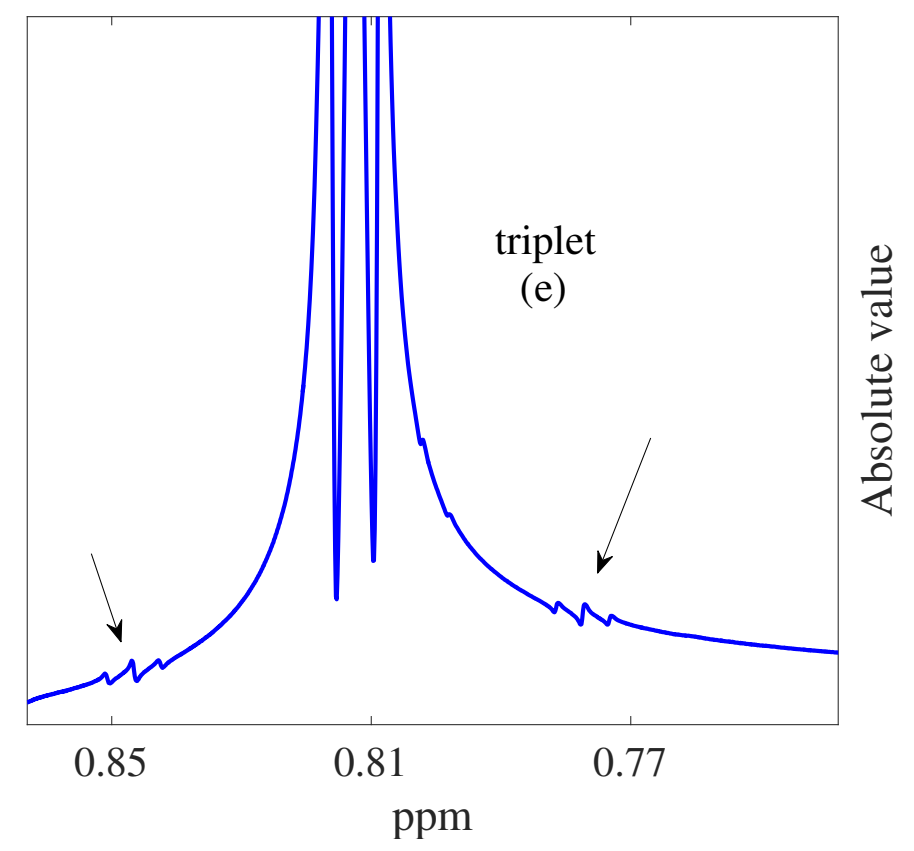

Figure 3.15: Carbon satellites in the vicinity of the triplet (e) of the pentanoic acid molecule.

Table 3.4 alongside those made without the inclusion of satellites. From Table 3.4, we observe that the satellites serve to increase the magnitude of the interaction terms, but this increase in their magnitude is comparable to the increase observed for the water peak. This can be explained by once again considering the bound expressions (3.63) and (3.64) for an individual peak. The magnitude term, i.e., the local term, in both (3.63) and (3.64) depends on the local properties of a peak, e.g., its damping factor. The interaction term, on the other hand, depends on the remote properties of the peak, i.e., the number of peaks in the spectrum, and how these peaks interact with the given peak. On close inspection of (3.65) and (3.66), it is revealed that the magnitude of these terms, which represents the magnitude of interaction, depends only on the damping factor and frequency of every other peak in the spectrum, but not on their amplitudes. This 
implies that the uncertainty introduced in the estimates of the peaks of interest by a large water peak or a small satellite peak will essentially be the same, given the frequency proximity and damping factor spread of both is same with respect to the given peak. This observation from Tables 3.3 and 3.4, leads us to make the following remark.

\section{A general remark on the impact of an extraneous peak on the estimation accuracy of the peaks of interest}

The uncertainty introduced in the estimation of parameters of a peak of interest due to extraneous peaks - whether solvent, carbon satellite or else - is independent of the extraneous peak amplitudes, and is proportional to their frequency proximity and the damping factor spread relative to the peaks of interest.

\subsubsection{Impact of second-order effects}

The spacing between adjacent peaks in a multiplet is known as the coupling constant. The coupled protons have the same coupling constant and their relative intensities follow a well-defined pattern predicted by Pascal's triangle [138]. Consequently, the multiplets should be symmetric, both in their amplitudes and the frequency spread. However, asymmetry is observed in the multiplets with respect to both amplitude and peak positions. The former, known as "leaning", causes peak amplitudes in a multiplet to depart from the ratios in Pascal's triangle. Due to the latter, peak positions no longer relate symmetrically. These effects are observed when chemical shift between the protons is smaller than the coupling constant between them, and are known, collectively, as the "second-order effects". This asymmetry in peak position leads to asymmetric magnitudes for the interaction terms computed for a given multiplet. This can be readily observed in Table 3.2. For example, for the MRS model, the unequal values of (3.66) are observed for peaks 1 and 3 of the triplet (b), i.e., 4.929 and 5.214 respectively. Similar asymmetry is observed in the values of (3.66) 
and (3.65) for pentuplet (c) and sextuplet (d). Consequently, any asymmetry in peak frequency positions in the multiplet will be readily mirrored in their interaction terms. On the other hand, asymmetry in the amplitude of multiplets would not be reflected in the interaction terms, as (3.66) and (3.65) do not depend on the amplitudes of the relative peaks. This, for an individual peak in a multiplet, will be reflected in the magnitude part of its bound for the frequency and damping factor, i.e., in (3.64) and (3.89), as an inverse relationship.

\subsubsection{Impact of baseline distortion}

A distortion in baseline of ${ }^{1} \mathrm{H}$ NMR spectra is manifested in the form of low frequency modulations [176]. A signal quantitation method observes these low frequency modulations as auxiliary peaks with very large damping factors. We know from our earlier discussion in Section 3.8.5 that addition of such auxiliary peaks to the spectrum of an FID signal increases the magnitude of the interaction terms for every peak, i.e., magnitude of (3.65) and (3.66). This, in turn, increases the bounds (3.63) and (3.64) and consequently the uncertainty associated with estimation of parameters of individual peaks. This increase in uncertainty, as noted in our earlier discussion in Section 3.7.1, becomes critical for parameter estimation of peaks with relatively small amplitudes. Accurate quantitation of these small peaks is crucial in some studies, e.g., in the study of metabolites where the error in the estimation of peaks can lead to the failure in identification of biomarkers characterising the signal [237].

\subsubsection{Impact of FID truncation}

Number of points in the ${ }^{1} \mathrm{H}$ NMR spectra is frequently increased by padding the time-domain FID data with zeros. This, however, serves to add an abrupt discontinuity to the original data if FID has not decayed, and causes the appearance of truncation artifacts in the FID spec- 
Table 3.3: Impact of water peak (MRS).

\begin{tabular}{llllll}
\hline & & \multicolumn{2}{c}{ With water peak } & \multicolumn{2}{c}{ Without water peak } \\
\hline & Peaks & $(3.65)$ & $(3.66)$ & $(3.65)$ & $(3.66)$ \\
Triplet & 1 & 31.5 & 6.5 & 43.8 & 7.7 \\
(e) & 2 & 212.9 & 20.1 & 267.5 & 22.5 \\
& 3 & 34.6 & 7.2 & 39.3 & 7.7 \\
\hline
\end{tabular}

Table 3.4: Impact of carbon satellites (MRS).

\begin{tabular}{llllll}
\hline & & \multicolumn{2}{c}{ With carbon satellites } & \multicolumn{2}{c}{ Without carbon satellites } \\
\hline & Peaks & $(3.65)$ & $(3.66)$ & $(3.65)$ & $(3.66)$ \\
Triplet & 1 & 61.7 & 15.8 & 25.1 & 5.2 \\
(b) & 2 & 368.8 & 27.9 & 82.7 & 11.4 \\
& 3 & 52.7 & 7.7 & 18.6 & 4.9 \\
\hline
\end{tabular}

trum [191]. These artefacts appear as undesired oscillation at the base of spectral peaks, and are viewed by the quantitation method as legitimate peaks to be characterised. This causes an increase the magnitude of interaction terms associated with every peak in the spectrum and the subsequent uncertainty associated with the estimation of their respective parameters. This effect will be pronounced for small peaks occurring in the proximity of larger peaks - the frequency proximity being directly translated to an approach towards singularity in (3.65) and (3.60) - and will serve to impair the estimation of their parameters.

\subsection{Conclusion}

In this chapter, analytical expressions are reported for the Cramer-Rao Lower Bound on the unbiased parameter estimates of all free-model parameters of the MRS model, the MRR model and the MRS model with 
previous knowledge. In addition, analytical expressions for the determinant and condition number of the FIMs of these models are also reported. Some auxiliary results, i.e., regarding the multiplicity of eigenvalues, the magnitude of the largest and smallest eigenvalues, and the factorisation of the characteristic polynomial associated with the respective FIMs of these models, were also presented. The derived expressions, when applied to real-time MRS data, were shown to successfully capture and account for various physical and chemical phenomena observed in spin-coupled MRS systems. 


\section{Chapter 4}

\section{Quantitation methods for 1D-NMR signals}

In this chapter, the state-of-the-art concerning the methods available for the quantitation of 1D-NMR FID data will be reviewed. Broadly, such methods can be categorised into:

- Time-domain methods, and

- Frequency-domain methods

\subsection{Time-domain methods}

Time-domain methods can be further sub-divided into [221]:

- Interactive methods, and

- Black-box methods

\subsubsection{Interactive methods}

These methods, requiring human interaction and input for their operation, have been termed as interactive methods. They try to obtain the maximum 
likelihood estimates of the FID parameters by first forming a cost function of residues and then minimising it. As the FID model is made up of complex damped exponentials (cisoids), the cost function is non-linear. As a result, the minimisation becomes a Non-Linear Least Squares (NLLS) problem [201]. This problem is particularly challenging to solve due to the difficulties that arise due to the inherent non-linearity of the cisoid model, e.g., ill-conditioning, local minima, etc [198]. In order to minimise this cost function effectively, the following four requirements need to be met [23]:

1. A prior estimate of the number of the cisoids, or model order,

2. A good initial guess of the parameters,

3. Convergence to an acceptable solution and in a reasonable time, and

4. Not getting stuck in local minima.

One way to meet the first requirement is to employ sub-space methods [50] or the Least Squares (LS)-based Prony methods [122]. However, due to the variable performance of these methods, it cannot be guaranteed that the initial guess generated by these methods will always be reasonable. Furthermore, these methods require manual input for the information about the model order in advance, which if not estimated correctly, introduces a systemic error in their results [141]. Nevertheless, keeping the requirements in view, the NLLS minimisation can be solved by using:

- Local optimisation search methods,

- Global optimisation search methods, and

- Specific methods

\section{Local optimisation methods}

Many of the search methods, stemming from Least Squares (LS) search problem, have been applied to the NLLS problem in order to search for 
the cost function minimum in the multidimensional parameter space. But these methods have the tendency to get stuck in local minima rather then finding the global minimum. This is their major drawback and prevents their application to the NLLS problem [79].

\section{Global optimisation methods}

The problem of getting caught in local minima has spurred the interest towards the development of more advanced search methods, termed as global optimisation search methods. Popular methods in this regard are simulated annealing, probabilistic simulated annealing, methods based on genetic algorithms, etc. These methods, in order to circumvent the local minimum problem, incur exceptionally high computational overhead and are sometime very slow [81, 149, 152,234,235].

\section{Specific methods}

Several methods, in their own regard, have become widely popular in the MRS community. The first of these widely used interactive methods was the Levenberg-Marquardt (LM) method which is a famous NLLS search method [146]. It is based on the Gauss-Newton (GN) algorithm and the Steepest Descent (SD) algorithm [92]. Though slower than the GN algorithm, it is much more robust regarding the first requirement for the search methods, i.e., it can converge to an acceptable solution even if the initial guess is not very accurate [162]. Another method to be applied to MRS is the Variable Projection for Non-linear Least Squares (VARPRO) method. It is a modified version of the LM method [106], and uses LM to minimise the variable projection VARPRO functional which, in turn, reduces the dimensionality of the search space [82]. Like the LM method, VARPRO is quite robust to the initial conditions as compared to other NLLS search methods. The drawback of VARPRO is that the computation of certain gradients, which it requires for its operation, can be very demanding computation- 
ally. However, this problem has been reported to be solved [163]. Next in line is the Advanced Method for Accurate, Robust, and Efficient Spectral Fitting (AMARES) [222]. In contrast to VARPRO, which minimises the variable projection VARPRO functional derived from the cost function, AMARES minimises the cost function itself. In doing so, AMARES offers much better performance in terms of robustness and accuracy. It is also much more flexible than VARPRO as it extends the possibilities of the incorporation of the prior information in the method. The possibility of choosing Gaussian peaks instead of Lorentzian peaks [222] has also been accommodated. Further in line is the Expectation Maximisation (EM) method [155]. The basic idea behind this method is to reduce the complexity of the high-dimensional search space by breaking it into many one-dimensional search spaces. Initially, this method did not allow the flexibility of incorporation of prior knowledge to facilitate the search process [137]. Later, this flexibility was also added [21].

\section{Advantages and disadvantages of interactive methods}

Overall, the interactive methods offer following advantages:

1. Flexibility in terms of peak fitting and incorporation of prior knowledge, and

2. If the assumption underlying the model and the noise are met, then the estimates obtained are the ML estimates.

Their drawbacks are that they

1. Are computationally inefficient,

2. Lack of automation as they require user interaction for their operation, e.g., for model order input. They also require user input for entering the starting values of the parameter estimates. This is because the local optimisation methods rely on a visual procedure, known as 
the peak-picking procedure, in order to obtain better starting values for the iterative methods [221],

3. No guarantee of finding the global minimum, and

4. No reasonable estimate of the time required to find the global minimum.

\subsubsection{Black-box methods}

These methods are termed Black-box methods because they are said to require minimal user input for their operation [221]. These methods can be sub-divided into four categories:

- Linear prediction based methods,

- State-space based method,

- Matrix pencil based methods, and

- Iterative Quadrature Maximum Likelihood Method

These methods are reviewed as follows.

\section{Linear prediction based methods}

These methods rely on the fact that FID model equation satisfies the equation for the Linear Prediction (LP) process. The LP equation, in turn, represents an Auto Regressive Moving Average (ARMA) model in terms of its Autoregressive (AR) and Moving Average (MA) parts . It is well-known that an ARMA model can be reasonably approximated by a high order AR model due to which the MA part of the LP equation is sometimes omitted [197]. As a result, the FID parameter estimation problem becomes an AR model parameter estimation problem. Two approaches have been traditionally employed to solve the latter; the first employs the Autocorrelation Function (ACF) of the received signal to construct the data matrix 
whereas the second employs the samples of the received signal directly. In MRS literature, the second approach is much popular than the first one. For example, the well-known LP Singular Value Decomposition (LPSVD) method [14] first performs the SVD of the data matrix containing the samples of the received signal, and then obtains a reduced-rank approximation to the data matrix by dropping the singular vectors corresponding to the singular values that lie below a certain threshold. After replacing the original data matrix by the reduced-rank one, a LS solution of the system is computed. By reducing the dimensionality of the data in this way, (i.e., removing data that does not contribute significantly to the reconstruction of the FID signal) the LPSVD method attempts to remove the noise from the FID signal. To further suppress the noise and hence improve the data fit, the Cadzow Enhancement Procedure (CEP) [56], which aims to restore the Hankel structure of the data matrix, can also be employed after the LPSVD, resulting in EPLPSVD method [55]. Furthermore, prior to the application of CEP, a minimum variance procedure was proposed [220] as a criteria for retaining the singular values in the rank-reduced matrix. Another possibility to remove the noise from data is to smooth it by performing Tikhonov regularisation (TR) which is applied in the LS stage instead of the rank-reduction process carried out by SVD. This forms the basis of the LPSVD Continuous Regularisation (LPSVDCR) method [114]. However, the LS step, i.e., the step performed on the reduced-rank matrix, assumes that the noise only exits in the received signal and not in the data matrix. This obviously is not true as the data matrix is also made up of the received signal with noise in it. The Total Least Squares (TLS) method [83] takes this point into account and, when applied after the rank reduction stage, has been shown to provide superior noise reduction as compared to the LS solution. TLS forms the basis for the next method, the LPTLS method [224]. 


\section{State-space based methods}

State-space based methods avoid the polynomial rooting process in LP based methods. They try to estimate the signal poles as the eigenvalues of the data matrix instead [172]. This is accomplished by creating the rankreduced matrix out of the Hankel data matrix using the SVD, deleting the extra rows at the bottom of the left singular vector matrix, and then computing the LS solution of the resulting system to obtain parameter estimates. The method that follows this process is known as Hankel SVD (HSVD) [224]. Another method, Hankel Lanczos SVD (HLSVD) is a computationally efficient variant of HVSD. It does not compute the full SVD of the data matrix but only part of it, using the Lanczos algorithm [153]. Another variant of the HVSD, known as the HTLS method [39], computes the TLS solution in HVSD instead of the LS solution in order to achieve better noise reduction and hence, more accurate estimates. Likewise in the LPSVD, the CEP has also been employed in HVSD and HTLS to improve noise reduction and result in better model fitting [40].

\section{Matrix pencil based methods}

The underlying principal of matrix pencil methods is very similar to that of state-space based methods. Both try to avoid the polynomial rooting process in LP based methods [228]. For the noiseless case, the estimates obtained by both are identical. For the noisy case, no significant difference has been reported between the estimates obtained by both methods [171]. Recently, a new localised matrix pencil method, termed as LocMaP [3], has been proposed for the parametric processing of high resolution NMR data.

\section{Iterative quadrature maximum likelihood method}

The Iterative Quadrature Maximum Likelihood Method (IQML) is an approximation of the ML method. It was proposed for obtaining "exact" 
maximum likelihood estimates of the parameters of superimposed exponential signals buried in noise [30]. However, it has been shown that frequency estimates obtained by IQML are "almost always inconsistent" [199].

\section{Advantages and disadvantages of black-box methods}

In general, the advantages of black-box methods are:

1. Require less user input compared with the interactive methods, i.e., being non-iterative methods they do not require a manual input for the starting values. However, they still require user interaction for model order input,

2. Are non-iterative. All estimates are computed in one go without the need for starting values,

3. Can incorporate prior knowledge,

4. Provide a good fit to data, and

5. Can represent non-Lorentzian peak shapes by a superposition of Lorentzian, Gaussian or Voigt shapes [15,98].

Their drawbacks are summarised as follows:

1. High computational complexity,

2. Limited flexibility for the incorporation of prior information,

3. Polynomial rooting can be time-consuming for large model orders, and

4. Estimates may be statistically suboptimal as the MA part of the ARMA model is not taken into account. 


\subsection{Frequency-domain methods}

In frequency-domain methods, the FID signal is first converted from the time-domain into the frequency-domain using the Discrete Fourier Transform (DFT) and then processed for the extraction of FID parameters. The methods then employed can be further sub-divided into two categories [151]:

- Peak-area integration methods, and

- Model fitting methods

\subsubsection{Peak-area integration methods}

These methods work by integrating the DFT spectrum of the FID signal. The signal, which is a sum of complex decaying exponentials (cisoids), has complex Lorentzian peaks associated with each cisoid in the frequencydomain. When phase aligned, the real part of a complex Lorentzian peak is always positive and has much narrower tails than its imaginary counterpart [109]. These methods rely on this observation and try to extract the information about the amplitude of the cisoid by calculating the area under the real peak. Integration is quite a straightforward approach and this is the main advantage of these methods.

\section{Advantages and disadvantages of peak-area integration methods}

Their advantages are listed as follows:

1. Simple and straightforward to implement,

2. Ideal for visual analysis, and

3. Computationally less demanding.

Below are their disadvantages [109]: 
1. Due to magnetic inhomogeneities, i.e., dipole-dipole interaction, Jcoupling, etc., the peaks may not be strictly of Lorentzian shape. Consequently, the estimates turn out to be biased,

2. Also since Lorentzian peaks are heavily tailed, the integration has to be truncated at some point which also introduces an error in the estimates [150],

3. In case of overlapping peaks, the integration approach is of little practical use,

4. These methods have limited resolution,

5. Baseline-distortion severely affects their performance. This distortion arises due to the unknown background in the FID spectrum and causes the baseline to be anything other than a flat line at zero intensity,

6. There is no systematic way to directly incorporate prior information,

7. The height of, and area under, the absorption component of a particular Lorentzian peak depends on the amplitude and the damping factor of the associated cisoid in time-domain. As a result, all frequency-domain methods focus on the extraction of peak amplitude information and leave out the damping factor which contains the information about $T_{2}$ [109],

8. These methods are inflexible in admission of peak shapes that arise due to magnetic inhomogeneities, i.e., Gaussian, Voigt, etc.,

9. They perform poorly at low SNR, and

10. Require perfect phasing. Ideally, at the start of the FID signal, magnitude of the absorption component is maximum and that of the dispersion component is minimum, i.e., zero. This, however, is not the 
case in general, and both components observe a certain phase shift. Consequently, neither the absorption nor the dispersion component has a perfect absorption mode line shape. This, in turn, does not yield a correct amplitude value if peak-area integration is performed for either of the components. Aprior phasing of the components is thus required before the integration can be correctly performed.

\subsubsection{Model-fitting methods}

Due to the many drawbacks associated with the peak-area integration methods, which prevent their direct application to the FID spectrum, more sophisticated methods, known as model fitting methods have evolved. There were particularly two reasons that motivated the development of these methods:

- Problem of overlapping peaks, and

- The problem of incorporation of the prior information

Basic idea behind these methods is this: Instead of integrating the area under each Lorentzian peak, try to fit a model - made up of complex Lorentzians - to the FID spectrum in the frequency domain. This process is similar to model fitting in the time-domain and hence follows the name, model-fitting methods. Afterwards, a NLLS algorithm, e.g., LM algorithm, is applied to extract the information about each cisoid, i.e., complex amplitude, frequency, and damping factor.

An early and popular method in this regard is the FITPLA method [6]. It uses the LM algorithm to find the maximum likelihood model parameter estimates by minimising the chi-square function between the frequency domain data points and the frequency domain model function. FITPLA is able to process both real and imaginary parts of the FID spectrum, can exploit prior knowledge during minimisation, and can also handle large FID data sets, which at that time, the time-domain methods like VARPRO 
could not handle due to the computationally demanding SVD [219]. The performance of this method is largely affected by the baseline distortion and hence, it requires baseline correction prior to its application. Another method based on the LM method is the QUALITY method [49]. This method can remove the effects of magnetic inhomogeneities on spectral peak shapes. Also, it can incorporate prior information during the process. But in quantitation of the spectra with overlapping peaks, this method requires additional frequency domain LS fitting in order to incorporate the prior information. Without the prior information, the method has been shown to lead to unreliable results even at high SNR [151]. Another model fitting method, that is specifically targeted for the spectral quantitation at low SNR, is termed as PIQABLE [158]. After the identification of peaks, this method performs peak smoothing prior to the calculation of peakareas areas using integration. But when applied to the overlapping peaks, this method has been pointed out to have obvious limitations. Yet another popular method in this domain is the Total Line Shape (TLS) method [193]. TLS has replaced the FITPLA method and provides more options in terms of incorporation of prior information, choice of initial guesses for the parameter estimates, and peak shape selection for model fitting. However, a crucial point on which the success of both FITPLA and TLS depends is that the spectral peaks should either have Lorentzian shapes or at least, a linear combination of Lorentzian shapes [124].

\section{Advantages and disadvantages of model-fitting methods}

Overall, model fitting methods offer the following advantages:

1. More flexibility in terms of prior information incorporation,

2. More flexibility in terms of peak shapes as they also allow the inclusion of Gaussian and Voigt peaks shapes, or a linear combination of these, in the specified model, 
3. Their estimates can be easily exploited as initial values for the NLLS methods, e.g., the LM method.

Their main drawbacks are enumerated as below:

1. Numerous corrections, in the form of pre-processing, have to applied to the FID spectrum before these methods can be applied. For example:

(a) Baseline-distortion needs to be corrected as it significantly degrades their performance,

(b) The deviation of the peak shape from the Lorentzian one, which primarily arises due to the magnetic inhomogeneities, should be accounted for, e.g., Gaussian and Voigt shapes have been used for that purpose [151], and

(c) Spectrum phasing is also an issue and crucial to the success of these methods [41].

\subsection{Time-domain vs frequency-domain methods}

Apart from many other drawbacks, one issue that severely limits the performance of the frequency-domain methods is the baseline distortion. In the absence of baseline distortion - which unfortunately cannot always be assumed - and given that the prior knowledge is incorporated, frequencydomain methods perform equally with the time-domain methods [218]. In some specific problems where the baseline distortion does not play a very significant role, frequency-domain methods can even be a better choice due to two reasons: their frequency selectivity and their computationally less demanding nature. However, these conclusions apply both to time and frequency-domain methods operating at high SNR. At low SNR, it has been ascertained that both perform equally poorly [26]. 


\subsection{Combined methods}

\subsubsection{Time-domain frequency-domain (TDFD) fitting}

One of the major drawbacks of the frequency-domain methods is that they suffer from the inflexibility for admitting various peak shapes that may arise due to magnetic inhomogeneities and other molecular phenomena. A method, known as the time-domain frequency-domain (TDFD) fitting [189] method, circumvents this problem by specifying the model in the time-domain. This allows the flexibility for admitting numerous peaks shapes. The fitting itself is performed in the frequency-domain using an iterative cost-function minimisation procedure. However, due to the timedomain to frequency-domain conversion required at each iterative step, the method turns out to be relatively slow.

\subsubsection{SVD-based frequency-selective methods}

SVD-based algorithms generally fall under the category of time-domain methods. Due to the large computational overhead incurred by the SVD, these methods are generally slow. This can be offset by combining them with the frequency-domain methods in the following way: once a frequency interval is picked by the frequency-domain method, only that particular region is then considered for parameter extraction in time-domain by the SVD-based method. As a result, much faster methods can be obtained in time-domain. A plethora of such methods exits in this domain as well, e.g., the SVD-based method usable in a SELected Frequency band (SELF-SVD), Method Of Direction Estimation with SELected Frequency band (SELF-MODE), the SVD-based High-Order Yule-Walker method in Sub-Bands (SB-HOYWSVD), etc [182]. However, the estimation results of these methods are largely dependent on the selection of appropriate frequency interval for processing, the location of spectral bands and their width. If not selected properly, the estimates in the individual spectral 
bands suffer from systematic bias and large variance [214].

\subsubsection{Method based on maximum likelihood and Bayesian analysis}

These methods try to find the maximum likelihood estimates of the FID parameters based on the Maximum Likelihood Method (MLM) and the Bayesian Analysis Method (BAM) [32]. The criteria used by both groups, the MLM and the BAM, is slightly different. The former tries to maximise the likelihood that the FID signal was generated by the given time-domain model. The latter maximises the posterior probability that the model is correct by incorporating a prior. In general, these methods work as follows. The FID signal is first augmented by zeros and then transformed to the frequency-domain. The largest amplitude spectral peak is selected and its parameters are extracted. By using the estimated parameters, the FID signal is reconstructed in time-domain using the damped cisoid model. This signal is then subtracted from the acquired FID signal in order to generate the residue. The residue is again transformed to frequency-domain and the subsequent largest peak is selected for further processing. This process is repeated until the signal is indistinguishable from noise. A method based on this approach is the Complete Reduction to Amplitude Frequency Table (CRAFT) [118]. In this method, a region-of-interest of the FID spectrum is first specified, which is filtered and downsampled to generate a sub-FID. BAM is then applied to convert the sub-FID into a table of amplitudes and frequencies. The performance results of this method, other than those presented by the authors in the original manuscript, have not been reported.

\subsubsection{Filter diagonalization method}

This is another high-resolution method for processing FIDs. The idea behind the Filter Diagonalization Method (FDM) is cast in its name. The 
filtering stage divides the spectrum into smaller subbands to reduce the computational overhead, and the diagonlization stage diagonalizes the matrices corresponding to these subbands for the extraction of individual subband parameters [142]. As this method endeavours to estimate the parameters of components lying in a pre-specified frequency band, this requires that the number of bases of FDM for a particular subband are properly chosen. In practice, this often necessitates a systematic analysis of the whole spectral band. This, without apriori information, further gives rise to the problem of selecting the spectral subbands, their width and their location, and the choices, if not carefully made, can strongly condition the resulting estimates [214].

These, and others issues highlighted throughout the chapter, shall be duly addressed in the subsequent chapter which reports the development of a high-speed, high-resolution and apriori-information-free algorithm for the quantitation of 1D-NMR signals. 


\section{Chapter 5}

\section{Quantitation algorithm for 1D-NMR signals}

In this chapter, we report the development of a high-speed, highresolution and apriori-information-free algorithm for the quantitation of 1D-NMR signals. The chapter commences with a formal introduction to the problem and gradually builds toward the proposed solution by highlighting the issues encountered. It culminates by presenting the results obtained by the algorithm on laboratory data which, when compared with the state-of-the-art in this area, demonstrate the superior performance of the proposed algorithm on all three fronts, i.e., speed, resolution and operation that is free of apriori information.

\subsection{Introduction}

Quantitation of a 1D-NMR FID signal, in general, requires the estimation of the number as well as the parameters of the cisoids constituting the FID signal, i.e., the complex amplitudes, damping factors, and frequencies, in a high-speed, high-resolution and aprior-information-free manner.

Many methods are available in the literature that attempt to address this task, e.g., methods based on maximum entropy, linear prediction, and 
state-space formulation $[78,126,224]$. Broadly, these methods can be subsumed under two categories: non-parametric and parametric.

Most popular of the non-parametric methods is the Fourier Transform (FT). This has been the most obvious approach for the estimation of FID parameters for a long time due to its relative ease and straightforward application. But the drawbacks of this approach are equally well-established, including limited resolution and large estimate variance [204].

Parametric methods (discussed in detail in Chapter 4) surpass their non-parametric counterparts in terms of resolution and sensitivity [23]. However, these methods suffer from systematic bias in their estimates due to the requirement that the number of cisoids in the FID signal model be known apriori. Furthermore, due to the use of the SVD, these methods can be computationally expensive for large data lengths.

The performance issues experienced by the FID quantitation methods can be attributed to the following [166]:

1. The number of peaks in the FID signal model is generally unknown, which, if adjusted by trial-and-error, generates a systematic bias in the estimates produced,

2. This lack of apriori information, when coupled with the possibility of a large number of peaks present in experimental FID signals, aggravates the influence of model order by increasing the possibility of false detections,

3. Low magnetic field strength reduces the SNR of the FID signal, which can adversely affect the performance of a quantitation method,

4. Low magnetic field strength also causes the spectra of the cisoids to overlap strongly, thus creating the issue of their accurate resolution,

5. The Nyquist sampling rate requirement for accurate resolution of peaks in FID signal can generate a large number of FID signal sam- 
ples, which not only increases the computational costs associated with the quantitation method (i.e., taxing its speed of operation), but also affects its quantitation performance for laboratory data sets containing a large number of dense, overlapping peaks,

6. The spectral dynamic range, i.e., the ratio of the largest to the smallest peak amplitude in the FID spectrum, can be high in experimental FID data. This results in occurrence of small peaks often embedded in the tails of relatively large peaks, making accurate resolution of the former challenging for the quantitation method, and

7. Magnetic field inhomogeneities and variations in the chemical environment increase the overall complexity of the FID signal

All these problems make the accurate extraction of parameters of FID signal much more complicated [31], and suggest the need for an alternative method that can cope with these issues in a robust manner. In order to rectify these issues, we suggest:

- The Steiglitz-McBride method (SM) for the quantitation of the FID signal: This method has been shown to exhibit superior performance over other methods when applied to the extraction of $T_{2}$ relaxation parameters for myelin water imaging of the brain - a problem that also employs an exponential signal model [23]. Also, it has been shown to be completely user-input free.

- A preprocessing stage of subband decomposition before the application of the SM method to the FID signal: This proposition solves the issues (1)-(6), because the subband decomposition results in [212]:

- An increase in the subband SNR,

- An increase in spectral resolution, and 
- A decrease in the length of the subband signals, making the execution of the SM method in individual subbands faster and serving to offset the additional computational overhead incurred by the subband decomposition process

- An adaptive subband decomposition process based on the Bayesian Information Criterion (BIC): This proposition addresses issues (1) and (2) by employing the BIC for model order selection. The BIC is selected due to its superior performance over comparable alternatives [202]. The BIC is also employed to make the subband decomposition process adaptive [58] and, accordingly, the proposed algorithm completely independent of user-input.

\subsection{Problem formulation}

Formal development of algorithm will be addressed in this section and henceforth.

\subsubsection{Signal model}

The FID signal in (2.3.1) is modelled as a sum of $N$ damped complex sinuosids (cisoids), being observed as $M$ samples, regularly spaced in time by $\Delta$ :

$$
r[m]=\sum_{n=1}^{N} \zeta_{n} e^{\left(-\alpha_{n}+j \omega_{n}\right) m \Delta}+v[m]
$$

where $m=0, \ldots, M-1, j=\sqrt{-1}$ and $\zeta_{n}=x_{n}+j y_{n}$ is the complex amplitude of the $n$-th component. $\alpha_{n}>0$ and $\omega_{n}$ are its decay rate and frequency, respectively. The noise process $v[m]$ is assumed white and complex normal, having zero mean and variance $\sigma^{2}$. We assume that sampling rate $1 / \Delta$ is sufficient for all frequency components to be adequately represented in the sampled signal. 


\subsubsection{Least square formulation}

The maximum likelihood estimate of $\left\{\zeta_{n}, \alpha_{n}, \omega_{n}, N\right\}$ is the Least-Square (LS) fit [201]:

$$
\min _{\left\{\zeta_{n}, p_{n}\right\}_{n=1}^{N}} \sum_{m=0}^{M-1}\left|r[m]-\sum_{n=1}^{N} \zeta_{n} p_{n}^{m}\right|^{2}
$$

with $p_{n}=e^{\left(-\alpha_{n}+j \omega_{n}\right) \Delta}$. However, the minimisation problem presented by (5.2) is non-linear and highly ill-conditioned. Moreover, $N$ is generally unknown [201].

\subsubsection{ARMA representation}

Under the assumption that $v[m]$ is white, $r[m]$ in (5.1) can be modelled as an Auto Regressive Moving Average (ARMA) process of the form [213]:

$$
r[m]=-\sum_{i=1}^{N} a[i] r[m-i]+\sum_{i=0}^{N-1} b[i] v[m-i]
$$

(5.3) is known as the minimal ARMA representation of $r[m]$. Information about the noiseless part of $r[m]$ is retained in the AR part of (5.2).

\subsection{Steiglitz-McBride algorithm}

The Steiglitz-McBride Algorithm (SMA), also called the Iterative Prefiltering (IPF) algorithm, was originally proposed for the computation of linear system LS parameter estimates. SMA achieves this by polynomial reparameterisation of (5.2) using (5.3) [203]:

$$
\min _{\{a[i]\}_{i=1}^{N},\{b[i]\}_{i=0}^{N-1}} \frac{1}{2 \pi} \int_{-\pi}^{\pi}\left|R(z)-\frac{B(z))}{A(z))}\right|^{2} d \omega
$$

with $B(z)$ and $A(z)$ being:

$$
B(z)=b[0]+b[1] z^{-1}+\cdots+b[N] z^{-N+1}
$$




$$
A(z)=1+a[1] z^{-1}+\cdots+a[N] z^{-N}
$$

with:

$$
\frac{B(z)}{A(z)}=\sum_{n=1}^{N} \frac{\zeta_{n}}{1-p_{n} z^{-1}}
$$

SMA computes the estimate of (5.6) by recursively solving the following equation:

$$
\min _{\{a[i]\}_{i=1}^{N},\{b[i]\}_{i=0}^{N-1}} \sum_{m=0}^{M-1}\left(\frac{R(z) A(z)-B(z)}{\hat{A}(z)}\right)^{2}
$$

where $\hat{A}(z)$ is the initial estimate, generally initialised to 1 , and $A(z)$ is the updated estimate of (5.6). The process is repeated until further improvement in the residue is not observed. Then $\hat{p}_{n}$ is computed from the roots of $\hat{A}(z)$. Finally, $\hat{\zeta}_{n}$ is obtained from:

$$
\hat{\zeta}_{n}=\left[\left(1-\hat{p}_{n} z^{-1}\right) \frac{B(z)}{\hat{A}(z)}\right]_{z=\hat{p}_{n}}
$$

\subsection{Model order selection}

Model order input is required in (5.8), and is generally unknown. The Bayesian Information Criteria (BIC) is considered for this purpose. It exhibits superior performance compared with alternatives available in this regard, i.e., Generalised Information Criterion (GIC), Akaike Information Criterion (AIC), etc [202]. The BIC, for optimal model order selection, adds an extra term to (5.2).

$$
\min _{\left\{\zeta_{n}, p_{n}\right\}_{n=1}^{N}} M \log \left[\sum_{m=0}^{M-1}\left|r[m]-\sum_{n=1}^{N} \zeta_{n} p_{n}^{m}\right|^{2}\right]+2 N \log (M)
$$

The extra term, serving as a penalty term, discards models with higher order if they do not achieve a significant reduction in the residue. This helps to prevent spurious estimates which do not contribute to reconstruction of $r[m]$. Also, this term does not incur significant computational cost. 


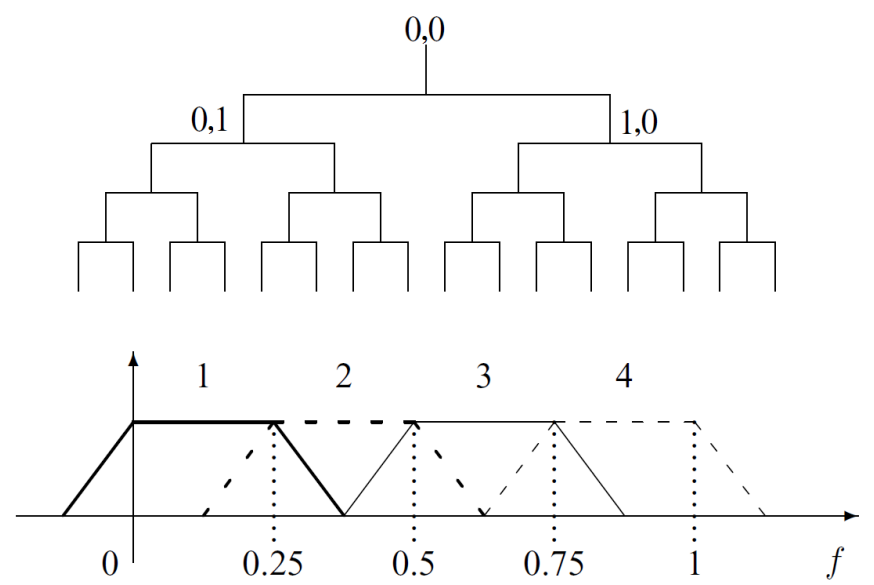

Figure 5.1: Illustration of the employed filterbank structure and the decomposition stages [58].

\subsection{Adaptive subband decomposition}

Subband decomposition converts (5.10) to numerous subband problems of lower model order. Advantages gained in individual subbands are: increased SNR, increased frequency resolution, reduced signal length, and reduced computational complexity [212]. Also achieved are advantages in speed and accuracy of the estimating algorithm when compared to the fullband problem. For this purpose, a uniform, multi-step subband decomposition is employed which successively filters and decimates the signal by a factor of 2 at every stage. Figure 5.1 illustrates this process along with the filterbank structure employed. Coiflets - due their ripple-free pass-band, sharp cut-off and relatively small group-delay - are used as decimation filters [226]. Decomposition yields the following decimated signal in individual subbands [60]:

$$
r^{q, s}(m)=\sum_{n=1}^{N^{\prime}} \zeta_{n}^{\prime}{ }_{n}^{\prime m}+v^{q, s}(m)
$$


for $m=0,1, \ldots, \hat{M}-1 . M^{\prime}$ is the number of signal samples in individual subbands, $N^{\prime} \leq N$ is the number of cisoids in the subband $(q, s)$, and $\zeta_{n}^{\prime}$ and $p_{n}^{\prime m}$ are parameter estimates obtained in the $(q, s)$-th subband. Once ${\hat{p^{\prime}}}_{n}^{m}$ are computed, their full-band versions can be obtained using:

$$
\begin{gathered}
\hat{p}_{n}^{m}=\left(\hat{p}_{n}^{\prime m}\right)^{1 / 2 q} e^{j \pi \frac{2 s+1}{2^{q}+1}} \quad \text { for } \omega \in[0, \pi] \\
\hat{p}_{n}^{m}=\left(\hat{p}_{n}^{m}\right)^{1 / 2 q} e^{j \pi\left(\frac{2 s+1}{2^{q}+1}-1\right)} \text { for } \omega \in[-\pi, 0]
\end{gathered}
$$

$\hat{\zeta}_{n}^{\prime}$ are computed from $\hat{\zeta}_{n}^{\prime}$ using (5.9). Since the responses of the decimation filters are not ideal, as depicted in Figure 5.1, their transition bands overlap. Consequently, a frequency component may be observed in several bands. The correct subband, i.e., the one to which the component actually belongs, can be identified using the following criteria [212]:

$$
\max \left|S_{q, s}\left(e^{j \hat{\omega}_{i}^{\prime}}\right)\right|
$$

where $\hat{\omega}_{i}^{\prime}$ is selected in the $(q, s)$-th subband with the highest amplitude in the corresponding band spectrum $S_{m}\left(e^{j \hat{\omega}_{i}^{\prime}}\right)$. In the final step, a BIC based stop criteria based on the frequency domain residual of the fullband signal is adopted to make the subband decomposition adaptive [58].

\subsection{Algorithm}

The complete process, in the form of an algorithm, is summarized as follows:

1. The signal is checked for poles using (5.10).

2. If poles are absent, algorithm is stopped.

3. Otherwise, pole parameters are extracted using SMA, and the residual is is subjected to subband decomposition.

4. Steps from (1) to (3) are repeated for individual subbands until a global halt is achieved. 
5. Subband estimates are converted to fullband using (5.12) and (5.13).

6. (5.9) is employed to compute $\hat{\zeta}_{n}$.

\subsection{Experimental results}

Experimental results obtained by the application of the proposed algorithm to real-time FID data are presented in this section.

\subsubsection{Acquisition/processing parameters}

The FID signals used in the demonstration of results were recorded on a 60 MHz spectrometer (Spinsolve Benchtop NMR; Magritek, Wellington, New Zealand). The length of the recorded signals was 32,768 samples. The signals were used "as-is" for algorithmic processing, i.e., without subjecting them to any phasing, filtering, correction or noise removal, etc. The proposed algorithm, as well as all other algorithms presented henceforth for comparison, were executed on an Intel $3.40 \mathrm{GHz}$ CPU.

\subsubsection{Comparison with SMA}

Figure 5.2 compares the spectrum of ethanol with the spectra generated from the parameter estimates obtained by the proposed algorithm and the SMA. As evident from the figure, the latter misses 10 out of 14 spectral peaks of ethanol whereas the proposed algorithm successfully captures all of them. Proposed algorithm, at the decomposition depth of 9, was able to achieve this at a comparable speed of 2.8 seconds when compared to 1.9 seconds taken by the SMA. 


\subsubsection{Comparison with LCModel}

For further elucidation, quantitation results obtained by the LCModel a software for automatic quantitation of ${ }^{1} \mathrm{H}$ NMR data and freely available ${ }^{1}-$ are also presented. These results along with those achieved by the proposed algorithm/SMA are displayed in Table 5.1. For each chemical sample, the FID signal was processed, and its spectrum was generated for comparison. Results presented include execution time, MSE between the true and reconstructed spectra, number of peaks missed/estimated in excess if any, and the decomposition depth utilised by the proposed algorithm. The results corroborate the earlier observation that the ex-

\footnotetext{
${ }^{1}$ http://s-provench-er.com
}

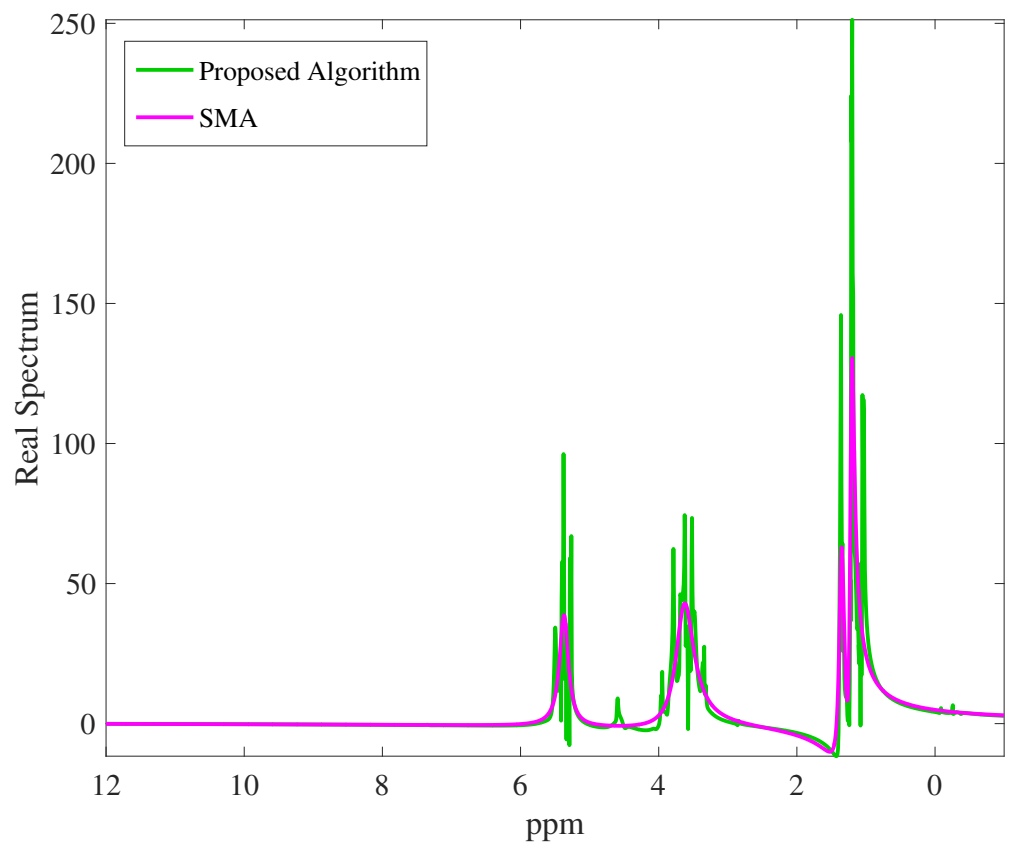

Figure 5.2: Comparison of regenerated FID spectra of ethanol to the original one. At this scale, no difference is visible between the true ethanol spectrum and that reconstructed using the proposed algorithm. 
ecution time incurred by the proposed algorithm is comparable to the SMA/LCModel.

Also observed is the number of spectral peaks missed by the LCModel /SMA becoming significant with increase in the complexity of chemical spectra. This is highlighted in the lactose spectrum, a challenging spectrum for a quantitation algorithm due to a large number of resonances (149), a high dynamic range $(90 \mathrm{~dB})$, and a noise floor with many resonances lying well below it. The ${ }^{1} \mathrm{H}$ spectrum for lactose, as well as the one estimated by the proposed algorithm, is displayed in Figure 5.3. The proposed al-

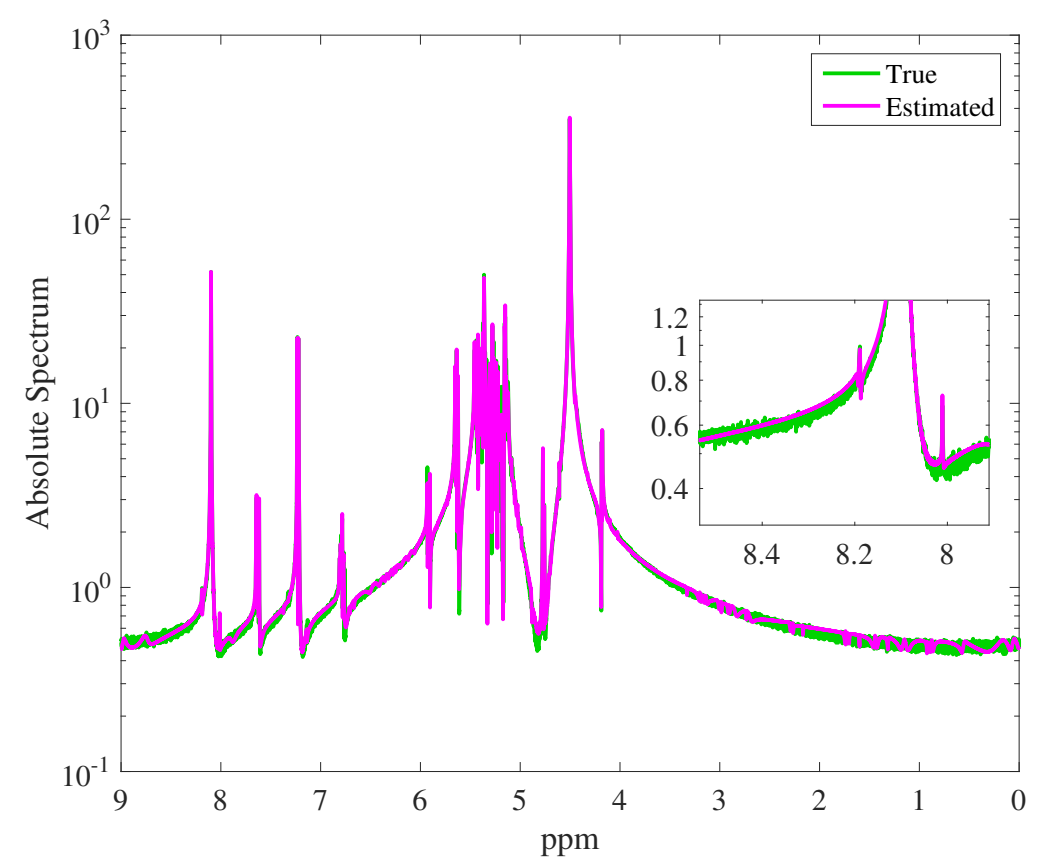

Figure 5.3: Comparison of the original and estimated ${ }^{1} \mathrm{H}$ spectrum of the lactose signal. Inset displays the zoomed view of the smallest peak captured by the proposed approach. This peak is as almost 35,000 times smaller than the largest peak in the signal. The proposed algorithm took just 5.7 seconds to capture all 149 peaks in the signal. The mean squared error achieved was $2.5 \times 10^{-5}$. 

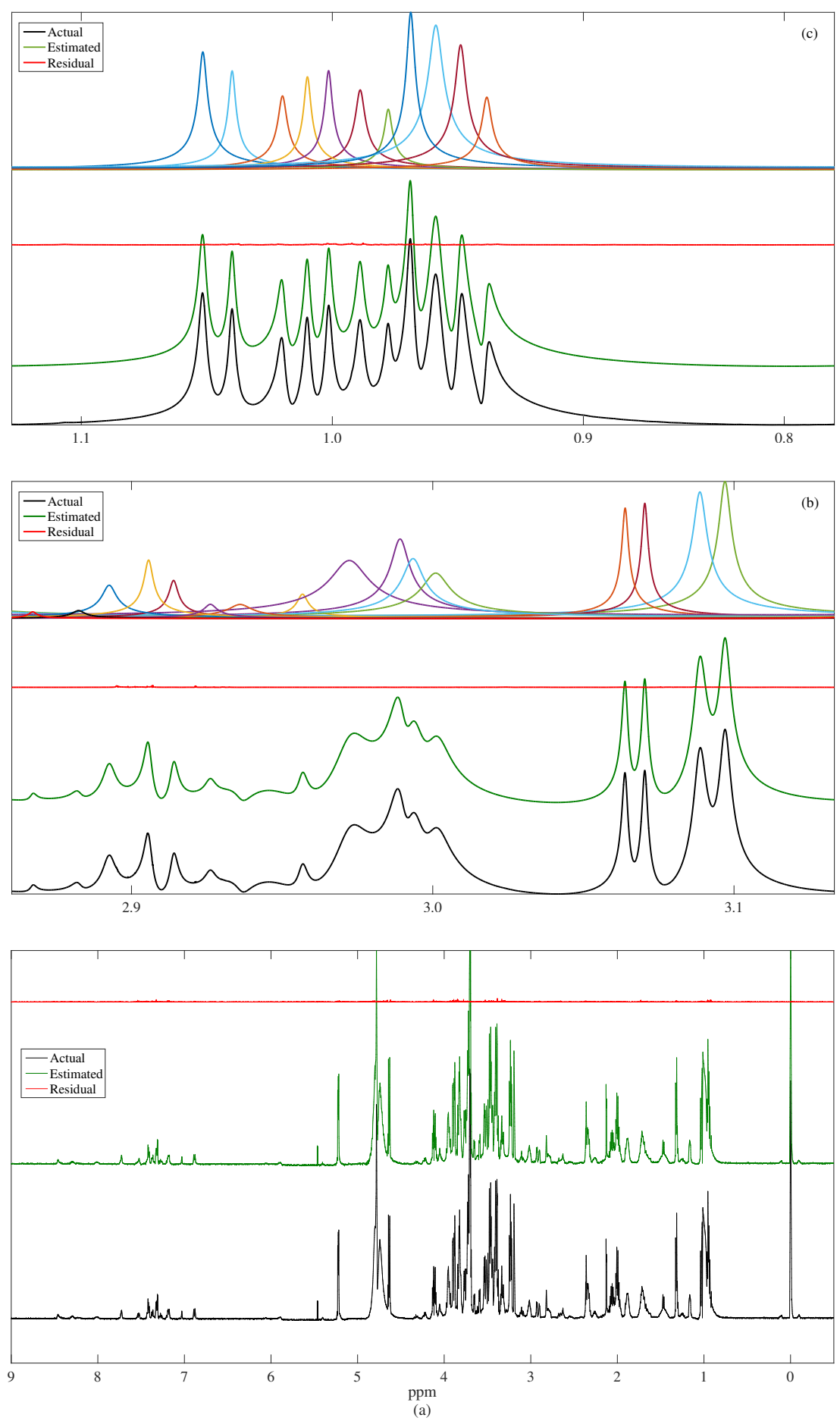

Figure 5.4: (a) Quantitation results achieved by the proposed algorithm for a representative fermentation broth in $90 \% \mathrm{H}_{2} \mathrm{O}$. The proposed algorithm took 12.4 seconds compared to 3.5 minutes taken by CRAFT [118], and does not require Regions of Interest (ROIs) to be specified. (b-c) Expansions of (a), displaying overlaid component spectra (from bottom to top, experimental, estimated, residual and component spectra). 


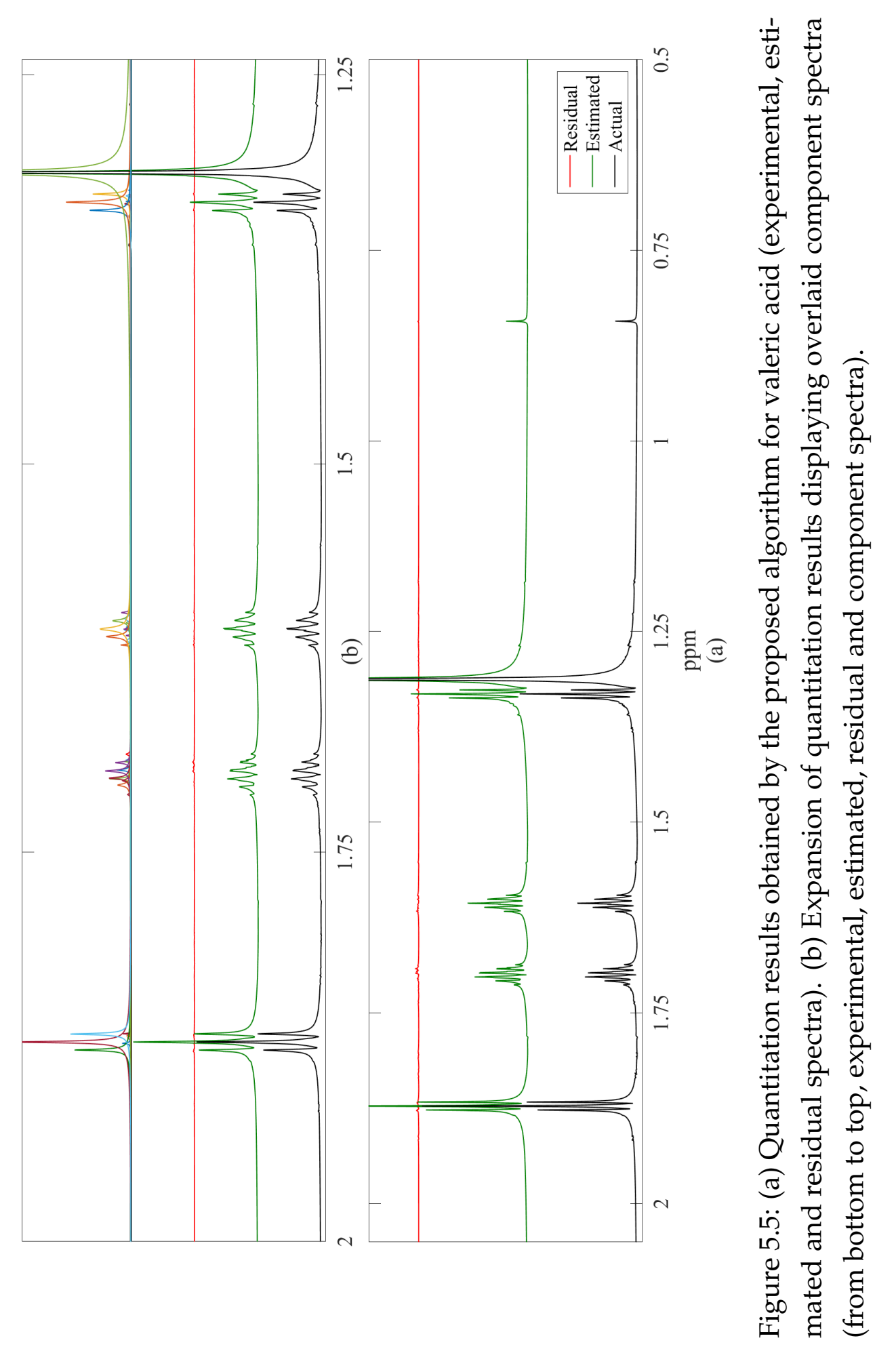


gorithm successfully estimated all spectral peaks - including the smallest peak which, also depicted in Figure 5.3, is almost 35, 000 times smaller than the largest peak. The algorithm took 5.7 seconds and achieved MSE of $2.5 \times 10^{-5}$. On the other hand, the SMA and the LCModel captured only 2 and 6 peaks out of 149 peaks, respectively.

\subsubsection{Comparison with CRAFT}

Finally, a performance comparison with Complete Reduction to Amplitude Frequency Table (CRAFT) - a Bayesian algorithm for quantitative NMR mixture analysis [118] - is presented. The CRAFT algorithm took approximately 3.5 minutes to analyze 28 Regions of Interest (ROIs) of ${ }^{1} \mathrm{H}$ NMR spectrum of representative fermentation broth in $90 \% \mathrm{H}_{2} \mathrm{O}$. The proposed algorithm took only 12.4 seconds to process the entire spectrum and, contrary to CRAFT, did not require specification of the ROIs; the true and estimated spectra are shown in Figure 5.4 which depict a close agreement. A similar close agreement is observed for the quantitation results obtained for the valeric acid (depicted in Figure 5.5).

\subsection{Conclusion}

In this chapter, a subband Steiglitz-McBride algorithm - based on the Steiglitz McBride algorithm, subband decomposition and the Bayesian Information Criterion - was presented for high-speed, high-resolution and apriori-information-free quantitation of 1D-NMR data. Proposed algorithm outperformed, on all three fronts, the previously published techniques in this area when applied to real-time 1D-NMR data of high complexity, i.e., with large data size, large number of overlapping resonances and a high-dynamic range. 


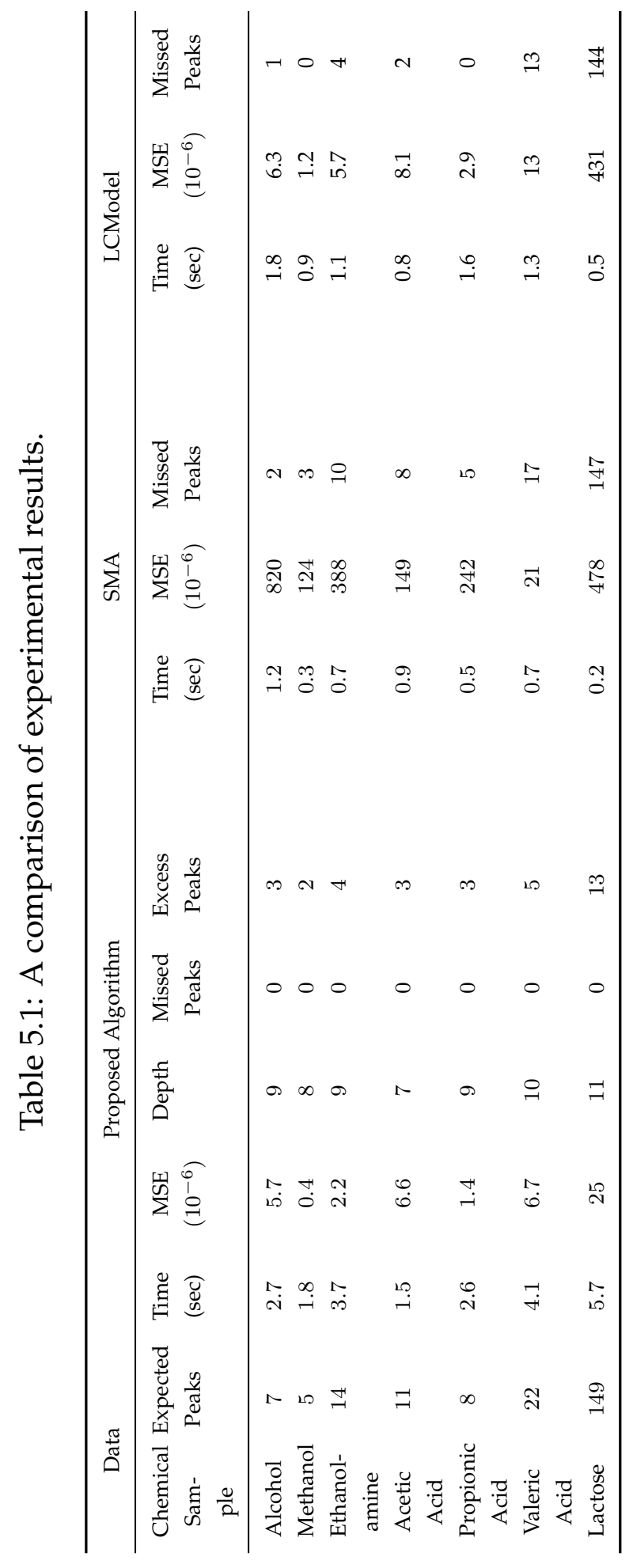


122CHAPTER 5. QUANTITATION ALGORITHM FOR 1D-NMR SIGNALS 


\section{Chapter 6}

\section{Quantitation methods for 2D-NMR signals}

In the discussions so far, a single FID signal has been the only source of information for the extraction of FID parameters, processed either in time or frequency-domain. However, a chemical sample may contain large number of peaks, many of which may overlap closely. Also, the ratio between the amplitude of the largest and the smallest peak in the sample can be high. As a result, small peaks often appear embedded in the tails of relatively large ones. This creates a marked difficulty in the analysis and extraction of chemical information from such spectra. In order to tackle this problem, two-dimensional (2D) NMR was proposed [139].

\subsection{Basic 2D NMR experiment}

The general scheme of a 2D-NMR experiment is depicted in Figure 6.1. The first stage is known as the preparation time in which the sample is excited by one or more pulses. The resulting magnetisation is allowed to evolve in the evolution stage; this time period forms the first time dimension $t_{1}$ know as the evolution time. Then the next stage, called the mixing stage, constitutes a further series of pulses allowing various molecu- 


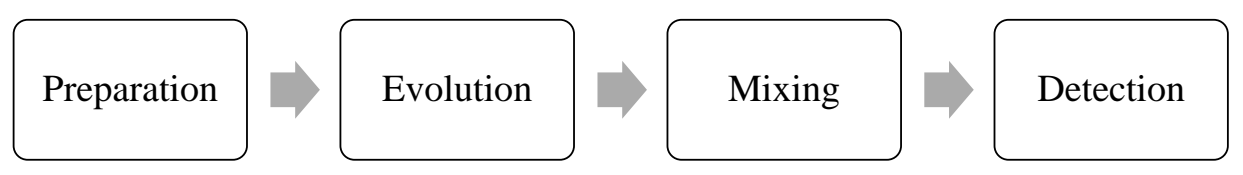

Figure 6.1: The general scheme of a 2D-NMR experiment.

lar couplings to interact. Finally, in the detection stage, the FID signal is recorded as a function of second time variable $t_{2}$ called the acquisition time. The resulting data is traditionally Fourier transformed with respect to the two variables $t_{1}$ and $t_{2}$ to obtain a $2 \mathrm{D}$ frequency spectrum which allows better resolution of closely overlapping peaks. The nature and amount of information yielded by the 2D spectrum depends on the exact nature of the pulse sequences [74].

\subsection{Types of 2D-NMR experiments}

In general, 2D-NMR experiments can be grouped into two categories [74]:

- Homonuclear 2D-NMR experiments,

- Heteronuclear 2D-NMR experiments, and

- Spatial 2D-NMR experiments

These categories, and the famous 2D-NMR experiments constituting them, are summarised below.

\subsubsection{Homonuclear 2D-NMR experiments}

In homonuclear experiments, the transfer of magnetisation occurs between nuclei of similar type during the evolution time. Popular 2D-NMR experiments in this category are discussed below. 


\section{COSY}

Correlation Spectroscopy (COSY) is probably the most popular 2D-NMR experiment, employed to resolve the coupling of closely-bound nuclei. In the COSY experiment, a mixing pulse is applied for the transfer of polarisation between spins that are directly J-coupled. The 2D frequency spectrum obtained from COSY experiment is known as the correlation map, and contains two types of peaks: diagonal and off-diagonal. Diagonal peaks, i.e., with identical value on both frequency axes, represent the contributions from nuclei for which the magnetisation did not change during the evolution process, and are the peaks to be expected in the 1D-NMR experiment. Off-diagonal peaks, on the other hand, indicate contribution from the nuclei that exchanged magnetisation during the evolution time and hence, depict interaction between the nuclei. Correlation is only observed if spin-spin coupling between the nuclei exists; otherwise, no coupling appears on the map.

\section{ECOSY}

Exclusive Correlation Spectroscopy (ECOSY), also known as long-range COSY, is tailored to resolve small-scale couplings. It accomplishes this by incorporating an additional delay of 100-400 milliseconds to the COSY pulse sequence [242]. This causes more relaxation to occur which in turn helps to ascertain if a certain coupling information existed which did not appear in the regular COSY spectrum.

\section{TOCSY}

Total Correlation Spectroscopy (TOCSY) is advantageous for elucidating coupling information from large, densely-coupled molecules. This is because TOCSY, in addition to resolving the coupling information between directly coupled nuclei, can also resolve the coupling information that ex- 
ists between nuclei in the same spin system. This is accomplished by inserting a series of repetitive pulses to allow for isotropic mixing during the transfer of magnetisation across a network of coupled atoms. Increased isotropic mixing times enable the magnetisation to span an increasing number of bonds.

\subsubsection{Heteronuclear 2D-NMR experiments}

In heteronuclear 2D-NMR experiments, the transfer of magnetisation occurs between nuclei of different types. Famous heteronuclear 2D-NMR experiments are outlined below.

\section{HSQC}

Heteronuclear Single Quantum Correlation Spectroscopy (HSQC) can detect coupling between nuclei separated by a single bond length. HSQC accomplishes this by employing pulse sequences with a particular delay that allows the detection of couplings for a range of given coupling constants. HSQC is particularly advantageous in enhancing the resolution of the $2 \mathrm{D}$ spectrum as it is able to suppress the multiplets that arise due to the ${ }^{1} \mathrm{H}$ couplings.

\section{HMBC}

Heteronuclear Multiple Bond Correlation Spectroscopy (HMBC) can detect coupling over multiple bond lengths. It does so by excluding delays from the HSQC pulse sequences which enables HMBC to cover the broad range of couplings resulting from multiple bonds.

\subsubsection{Spatial 2D-NMR experiments}

These experiments considers the magnetization effects that arise due to nuclei being in close spatial proximity to each other, regardless of the exis- 
tence of a bond between them. Famous 2D-NMR experiments are outlined below [80].

\section{NOESY}

Nuclear Overhauser Effect Spectroscopy (NOESY) is the most popular among the spatial 2D-NMR experiments. In order to neutralise magnetisation effects arising due to the spatial proximity of nuclei, NOESY employs a different pulse sequence to that employed in homo/heteronuclear experiments. After the application of the first pulse and then waiting for the evolution time, a second pulse consisting of two closely-spaced pulses in time is applied. The first pulse, similar to that in COSY, eliminates the effects of bond coupling whereas the second pulse neutralizes the effect of spatial coupling. Afterwards, the FID is collected over acquisition time $t_{2}$. NOESY experiments are helpful in stereochemistry, i.e., identification of the three-dimensional arrangement of atoms and molecules.

\section{ROESY}

The Nuclear Overhauser Effect (NOE) accounts for the change in the magnitude of the peaks of a particular nucleus when a nucleus in its proximity is stimulated by an RF pulse. In NOESY, NOEs are positive when accumulated for small molecules, and negative for large molecules. Rotatingframe Nuclear Overhauser Effect Spectroscopy (ROESY) peaks, on the other hand, are always positive. This is accomplished by spin-locking the equilibrium magnetisation so that it cannot precess. ROESY, hence, can obtains NOE information about a molecule without previous knowledge of its spectral assignment or structure. 


\subsection{Applications of 2D NMR}

2D NMR spectroscopy is particularly important in structural biology where it is used to determine the structures of proteins [236]. This is primarily due to the ability of 2D NMR to resolve overlapping peaks. Also, it is rapidly finding new applications in metabolomics [17], e.g., for the detection of metabolites, and pharmaceuticals [99] for chemical characterisations.

\subsection{Practical issues of 2D-NMR}

As observed from the preceding discussion, adding an extra dimension to the NMR experiment can help to resolve overlapping peaks. Also, it can facilitate the extraction of complex molecular information. However, this raises two issues of practical concern: the issue of acquisition time and the limitations imposed by the FT.

\subsubsection{Limitations imposed by the acquisition time}

Acquisition time for an NMR experiment rises exponentially with every added dimension [47]. This sets a practical limit to the number of dimensions in which the NMR data can be collected. Table 6.1 shows a comparison of the acquisition time required for multidimensional NMR experiments [47]. As observed from the Table 6.1, the acquisition time for a 2D-NMR experiment is around 64 minutes as compared to 30 seconds required for 1D-NMR experiment, representing a 128 fold increase over the former. To further highlight this limitation, also shown in Table 6.1 is the acquisition time for a 3D-NMR experiment which can be as large as 136 hours. This is almost equivalent to 6 days, and is impractical for lab data acquisition purposes. Consequently, the acquisition time sets a limit to the number of data points available in a 2D-NMR experiment. 
Table 6.1: Comparison of acquisition time for multidimensional NMR experiments [47].

\begin{tabular}{cc}
\hline Dimensions of NMR experiment & Data acquisition time \\
\hline $1 \mathrm{D}(1$ FID $)$ & 30 seconds \\
$2 \mathrm{D}(128$ FIDs $)$ & 64 minutes \\
$3 \mathrm{D}(128 \times 128$ FIDs $)$ & 136 hours \\
\hline
\end{tabular}

\subsubsection{Limitations imposed by FFT}

The time-domain data acquired from a 2D-NMR experiment is traditionally fast Fourier transformed to the frequency-domain for spectral processing. The FFT is applied in both $t_{1}$ and $t_{2}$ dimensions. Two issues arise immediately:

- The use of the FFT on NMR data creates an unavoidable resolution/sensitivity discrepancy inherent to FFT, and

- This is compounded by relatively fewer data points in the coupling dimension, a direct consequence of the limitation imposed by the acquisition time on multidimensional NMR experiments

Number of the points in the FFT spectrum can be increased by adding zeros to the end of the time-domain FID data. This, however, serves to add an abrupt discontinuity to the original data and causes the appearance of artefacts in the spectrum, known as truncation artefacts [191].

These artefacts can be circumvented by gradually reducing the amplitude of the FID signal at the point of truncation, a process known as "apodization". This process, though it improves the resolution without the appearance of additional artefacts, ends up in broadening the spectral peaks, thereby reducing the sensitivity of the signal. 
Another way to improve the length of the FID spectrum is to sample the FID for longer time periods. This generates larger data sets and hence, better resolution. However, this is impractical for multidimensional NMR experiments because, as indicated in Table 6.1, the acquisition of additional data points tremendously increases the acquisition time of the experiment [154].

\subsection{Addressing the limitations}

Approaches taken to overcome the aforementioned issues can be grouped in two categories:

- Pre-acquisition methods, and

- Post-acquisition methods

\subsubsection{Pre-acquisition methods}

These methods, known as pre-acquisition methods, employ techniques other than the evolution time to extract correlation maps in multiple dimensions. They either constitute modification of pulse sequences prior to the acquisition of the FID signal and/or any other hardware changes [154]. These, being hardware-based methods, lie beyond the scope of this thesis.

\subsubsection{Post-acquisition methods}

Post-acquisition methods modify the signal after its acquisition. They employ high-resolution signal processing methods that focus on extraction of information from short or incomplete data records without requiring them to be sampled over longer time periods. They are generally softwarebased methods, and currently form the centre of research activity to overcome the restrictions imposed by the FFT. They are also in line with the 
research carried out in this thesis and will be the focus of attention henceforth. These methods can be split into two categories according to the sampling schemes they employ:

- Non-uniform sampling methods, and

- Uniform sampling methods

\subsection{Non-uniform sampling methods}

These methods employ non-uniform sampling techniques in the timedomain and can be further split into [107]:

- Deterministic sampling methods, and

- Irregular sampling methods

\subsubsection{Deterministic sampling methods}

Deterministic sampling methods use deterministic sample distributions in time-domain to sample outside the grid defined by the Nyquist theorem. A powerhouse of such schemes is one that employs radially sampled data [25]. It generates radial projections of data based on the so called Projection-Slice theorem [28]. The theorem states that the FFT of the data recorded along a radial axis in the time-domain is equivalent to the projection of the $2 \mathrm{D}$ spectrum on the same axis in frequency-domain. Once collected, these projections are analysed individually by numerous postprocessing methods discussed in Chapter 4 to locate the position of peaks in the spectrum. This approach effectively reduces the dimension of the $2 \mathrm{D}$ experiment by one wherein the individual projections can be processed to obtain the results of the full $2 \mathrm{D}$ experiment. An issue with this approach is that the data in different dimensions turns out to be dependent; peaks in different dimensions are linked, giving rise to multiplets, in which the 
number of peaks observed in a lower dimension depend on the number of dimensions that are linked. As a result, deconvolution procedures need to be applied prior to the extraction of any useful information. The utility of this approach for practical NMR applications, e.g., for extraction of information about the relaxation rates, is still under investigation [154].

\subsubsection{Irregular sampling methods}

These methods employ irregular sampling schemes, also known as NonUniform Sampling (NUS) schemes, to minimise the number of data points recorded in a particular dimension [107]. A general way of doing this is to employ a random distribution - Gaussian, Poisson, etc. - to locate the time scale for sample collection. The data thence recorded lies outside the Nyquist grid. Consequently, these methods impose another requirement: the data processing method should be able to generate the spectrum of the signal from its irregularly spaced samples. Direct application of FFT to such data is equivalent to leaving certain basis functions out of the FFT formula. As a result, its orthonormal basis set is no longer complete and the remaining basis functions interfere with each other. This can also be viewed as the convolution of the spectrum of the uniformly sampled signal and that of the sampling function. The latter is responsible for generating associated sampling artefacts [185]. Removal of these artefacts from the spectrum is an issue of significant contemporary interest and a motivation behind the development of non-Fourier based methods.

\subsection{Uniform sampling methods}

These methods endeavour to overcome the limitations posed by acquisition time/FFT by exploiting alternative approaches directly applicable to the uniformly sampled data. Often they are the extensions of 1D-NMR quantitation methods to 2D, e.g., 2D MUSIC [132], 2D IQML [44], 2D ES- 
PRIT [179], TLS-Prony [180], 2D Matrix Pencil [103], etc.

Another major family of techniques employs Linear Prediction (LP) for Autoregressive (AR) modelling of 2D-NMR data: Linear Predictive Singular Value Decomposition (LPSVD) [243], Linear Predictive QR Decomposition (LPQRD) [210], Linear Prediction Maximum Entropy Method (LPMEM) [197], etc.

Yet another category, known as SVD-based high resolution methods, includes: Hankel SVD (HSVD) [140], Matrix Enhancement Matrix Pencil (MEMP) [96], Multi-Dimensional Folding (MDF) [136], Improved MultiDimensional Folding (IMDF) [134], and Principal singular vector Utilization for Modal Analysis (PUMA) [192].

These subspace/linear prediction/SVD-based approaches have been shown $[57,181,240]$ to suffer from performance issues as well as high computational costs - incurred by extensive computations in polynomial rooting/matrix decomposition procedures - when applied to high complexity real-time 2D-NMR signals, i.e., with a large number of data samples and/or cisoids. 
134 CHAPTER 6. QUANTITATION METHODS FOR 2D-NMR SIGNALS 


\section{Chapter 7}

\section{Quantitation algorithm for 2D-NMR signals}

In Chapter 4, we demonstrated that the Steiglitz-McBride algorithm, when used in conjunction with the subband decomposition process and the BIC, yields excellent quantitation results on 1D-NMR data in terms of speed, accuracy and apriori-information-free operation. In this chapter, we demonstrate that the 2D Steiglitz-McBride algorithm, along with 2D subband decomposition and the 2D-BIC, permits fast, accurate and automated extraction of quantitation information from 2D-NMR data. The proposed algorithm is able to process the time-domain 2D-NMR data directly, and does not require linear prediction, baseline and phase correction, zero-filling, or apodization for preprocessing. This makes the algorithm favourable for direct application to time-domain data from 2D-NMR experiments, which is verified by the application of the proposed algorithm to a variety of both homo- and heteronuclear experimental data. The results demonstrate that the proposed algorithm clearly outperforms the previously published methods in this area in terms of speed, accuracy and operation free of apriori input. 


\subsection{Introduction}

Comprehensive molecular analysis using 2D-NMR spin interactions requires lengthy experimental measurements for the acquisition of the necessary spectral information. The measurement time for a multidimensional NMR experiment rises exponentially with every added dimension, and sets a practical limit to the number of dimensions in which the NMR data can be collected [47]. Consequently, 2D-NMR data is almost invariably truncated, especially in the indirect dimension, i.e., the extra dimension added by the 2D-NMR experiment. This hampers the use of 2D-NMR in recent high-throughput applications [90].

The necessity for prolonged measurement times for a 2D-NMR experiment has been attributed to, and eventually characterised into two regimes: sensitivity-limited and sampling-limited [131]. The former, due to inherently low sensitivity of 2D-NMR experiments, relies on increased measurement times to obtain higher Signal-to-Noise (SNR) ratio. The latter, in order to achieve a specific spectral resolution, employs a certain measurement time dictated by the Nyquist sampling rate. Efforts have been directed towards retaining a high spectral resolution while keeping the measurement time minimal.

Fourier transformation (FT) has been traditionally employed for postprocessing of the truncated 2D-NMR data due to its relative ease and straightforward application. Use of the FT for truncated data-sets suffers from unavoidable resolution/sensitivity trade-off inherent to the FT. To overcome this issue, Linear Prediction (LP) has been widely used prior to FT processing. Methods employing LP for 2D-NMR data processing include: Linear Predictive Singular Value Decomposition (LPSVD) [243], Linear Predictive QR Decomposition (LPQRD) [210], and the Linear Prediction Maximum Entropy Method (LPMEM) [197]. The use of LP for processing of 2D-NMR data necessitates a 2D deconvolution operation before the quantitation of 2D frequency-domain data can be performed by FT. 
Quantitation by FT, in turn, requires peak integration/fitting which relies on rigorous apriori phase/baseline correction. A detailed discussion of the limitations of LP and FT for 2D-NMR data processing has been carried out in [175] and [140] respectively.

Lately, mixed time-frequency methods, such as the Complete Reduction to Amplitude Frequency Table (CRAFT) [119], have been proposed as an alternative to FT for processing 2D-NMR data. CRAFT, inherently, is a 1D method [118]. It is applied to 2D data in two steps. FT is performed along the direct dimension after zero-filling, followed by phase-correction. In the second step, corresponding to each frequency in the direct dimension, time-domain data in the indirect dimension is extracted, apodized, and processed using CRAFT. Consequently, peak-width is dominated by the apodization function. A method using a similar approach is Absolute Minimal Sampling (AMS) [90] which, in turn, is a generalisation of Singlepoint Evaluation of the Evolution Dimension (SPEED) [123]. AMS and SPEED use a non-linear least-squares procedure for fitting time-domain data in the indirect dimension.

Numerous other methods have been proposed to improve the spectral resolution of truncated data sets. Many employ LP for Autoregressive (AR) modelling of 2D-NMR data: Linear Predictive Singular Value Decomposition (LPSVD) [243], Linear Predictive QR Decomposition (LPQRD) [210], Linear Prediction Maximum Entropy Method (LPMEM) [197], 2D Filter Diagonalization Method (FDM) [142], etc. Yet another category, known as SVD-based high resolution methods, includes: Hankel SVD (HSVD) [140], Matrix Enhancement Matrix Pencil (MEMP) [96], Multi-Dimensional Folding (MDF) [136], Improved Multi-Dimensional Folding (IMDF) [134], and Principal singular vector Utilisation for Modal Analysis (PUMA) [192].

Though these extension/SVD based methods offer better resolution and detection properties than FT, they suffer from several drawbacks. Firstly, they have been shown to experience performance issues when ap- 
plied to real-time 2D-NMR signals with high complexity, i.e., with relatively large data size, large number of dense, overlapping modes, and possibly, low SNR [57]. Secondly, computational costs associated with these methods can be prohibitive due to the extensive computations required in their polynomial rooting and matrix decomposition procedures [240]. Thirdly, these methods generally require apriori information about the number of modes in data which, if adjusted by trial-and-error, not only generates a systemic bias in their output, but also prevents operation that is independent of user input [59].

Application of subband decomposition prior to the quantitation of 2DNMR data can help in resolution of these and other highlighted issues as follows [212]:

- It improves SNR in individual subbands, which serves to offset the inherent low sensitivity of 2D-NMR experiments,

- It increases spectral resolution, which enables improved spectral quantitation of truncated data sets arising from limited measurement time, and

- It reduces the data size in individual subbands, which helps to alleviate the computational load on the quantitation method involved.

Lastly, in order to enable a user-input independent operation, the Bayesian Information Criterion (BIC), due to its superior performance when compared to the alternative methods available [202], can be employed for the estimation of number of modes.

\subsection{Problem formulation}

This section presents the formal introduction to the problem and establishes the framework for its solution. 


\subsubsection{The 2D signal model}

A two-dimensional Free Induction Decay (FID) signal $r\left(m_{1}, m_{2}\right)$ can be modelled as a sum of $N$ two-dimensional damped complex sinusoids (cisoids), being observed as $M_{1} \times M_{2}$ samples, regularly spaced in time by $\Delta_{1}$ and $\Delta_{2}$ respectively: [132]:

$$
r\left(m_{1}, m_{2}\right)=\sum_{n=1}^{N} \zeta_{n} e^{\left(-\alpha_{n}^{(1)}+j \omega_{n}^{(1)}\right) \Delta_{1} m_{1}+\left(-\alpha_{n}^{(2)}+j \omega_{n}^{(2)}\right) \Delta_{2} m_{2}}+v\left(m_{1}, m_{2}\right)
$$

where $\zeta_{n}=\beta_{n} e^{j \theta_{n}}$ is the complex amplitude of the $n$-th 2D cisoid, and $\omega_{n}^{(1)}, \omega_{n}^{(2)}$ and $\alpha_{n}^{(1)}, \alpha_{n}^{(2)}>0$ are its frequencies and damping factors corresponding to two sampling dimensions $m_{1}$ and $m_{2}$ respectively, with $m_{1}=0, \ldots, M_{1}-1, m_{2}=0, \ldots, M_{2}-1$ and $j=\sqrt{-1} . v\left(m_{1}, m_{2}\right)$ is complex two-dimensional Gaussian white noise. Sampling rates $1 / \Delta_{1}, 1 / \Delta_{2}$ are assumed sufficient for all frequency components to be adequately represented in the sampled signal. It is not assumed that $M_{1}$, nor $M_{2}$, is sufficiently high for the signal to have faded by the $M_{1}$-th and $M_{2}$-th sample, i.e., $\left\{M_{l} \Delta_{l} \alpha_{n_{l}} \ngtr 1 \forall n\right\}_{l=1}^{2}$. The noise free version of $r\left(m_{1}, m_{2}\right)$ is denoted by $\hat{r}\left(m_{1}, m_{2}\right)$ :

$$
\hat{r}\left(m_{1}, m_{2}\right)=\sum_{n=1}^{N} \zeta_{n} p_{n}^{m_{1}} q_{n}^{m_{2}}
$$

with $p_{n}=e^{\left(-\alpha_{n}^{(1)}+j \omega_{n}^{(1)}\right) \Delta_{1}}$ and $q_{n}=e^{\left(-\alpha_{n}^{(2)}+j \omega_{n}^{(2)}\right) \Delta_{2}}$.

\subsubsection{Least square formulation}

The problem is to estimate the number $N$ of the $2 \mathrm{D}$ cisoids as well as their associated parameters $\left\{\zeta_{n}, p_{n}, q_{n}\right\}_{n=1}^{N}$. This estimation problem can be modelled as the least square (LS) fitting procedure:

$$
\min _{\left\{\zeta_{n}, p_{n}, q_{n}\right\}_{n=1}^{N}} \sum_{m_{1}=0}^{M_{1}-1} \sum_{m_{2}=0}^{M_{2}-1}\left|r\left(m_{1}, m_{2}\right)-\hat{r}\left(m_{1}, m_{2}\right)\right|^{2}
$$


This minimisation problem is nonlinear and ill-conditioned. Moreover, $N$ is generally unknown.

\subsubsection{ARMA modelling}

Taking the 2D $z$-transform of (7.2) yields:

$$
\hat{R}\left(z_{1}, z_{2}\right)=\sum_{n=1}^{N} \frac{\zeta_{n}}{\left(1-p_{n} z_{1}^{-1}\right)\left(1-q_{n} z_{2}^{-1}\right)}
$$

Expanding the partial fractions in (7.4) leads to the following Auto Regressive Moving Average (ARMA) representation of $\hat{r}\left(m_{1}, m_{2}\right)$ :

$$
\hat{R}\left(z_{1}, z_{2}\right)=\frac{B\left(z_{1}, z_{2}\right)}{A\left(z_{1}, z_{2}\right)}
$$

with $B\left(z_{1}, z_{2}\right)$ and $A\left(z_{1}, z_{2}\right)$ given by:

$$
\begin{array}{r}
B\left(z_{1}, z_{2}\right)=b(0,0)+b(1,0) z_{1}^{-1}+b(0,1) z_{2}^{-1}+\cdots+b(N-1, N-1) z_{1}^{-N+1} z_{2}^{-N+1} \\
A\left(z_{1}, z_{2}\right)=1+a(1,0) z_{1}^{-1}+a(0,1) z_{2}^{-1}+\cdots+a(N, N) z_{1}^{-N} z_{2}^{-N}
\end{array}
$$

The AR part of (7.5) is sufficient for estimation of the damping factors and frequencies.

\subsection{D Steiglitz-McBride algorithm}

The 2D Steiglitz-McBride Algorithm (2D-SMA), also called the 2D Iterative Prefiltering algorithm, uses the ARMA representation in (7.5) to reparameterise (7.3) [133]:

$$
\min _{\left\{a\left(k_{1}, k_{2}\right)\right\}_{k_{1}, k_{2}=1}^{N},\left\{b\left(k_{1}, k_{2}\right)\right\}_{k_{1}, k_{2}=0}^{N-1}} \frac{1}{(2 \pi)^{2}} \int_{-\pi}^{\pi} \int_{-\pi}^{\pi}\left|E\left(z_{1}, z_{2}\right)\right|^{2} d \omega_{1} d \omega_{2}
$$

with

$$
E\left(z_{1}, z_{2}\right)=R\left(z_{1}, z_{2}\right)-\frac{B\left(z_{1}, z_{2}\right)}{A\left(z_{1}, z_{2}\right)}
$$


The 2D-SMA estimates $A\left(z_{1}, z_{2}\right)$ by iteratively minimising the residual $E\left(z_{1}, z_{2}\right)$ in $(7.8):$

$$
E^{(i+1)}\left(z_{1}, z_{2}\right)=\frac{R\left(z_{1}, z_{2}\right) A^{(i+1)}\left(z_{1}, z_{2}\right)-B^{(i+1)}\left(z_{1}, z_{2}\right)}{A^{(i)}\left(z_{1}, z_{2}\right)}
$$

where $A^{(i+1)}\left(z_{1}, z_{2}\right)$ and $B^{(i+1)}\left(z_{1}, z_{2}\right)$ are the updated estimates of $A^{(i)}\left(z_{1}, z_{2}\right)$ and $B^{(i)}\left(z_{1}, z_{2}\right)$ respectively. The method can be formulated by defining:

$$
H^{(i)}\left(z_{1}, z_{2}\right)=\frac{1}{A^{(i)}\left(z_{1}, z_{2}\right)}
$$

which lends the signal domain representation of (7.10) to be:

$e^{(i+1)}\left[m_{1}, m_{2}\right]=r^{(i)}\left[m_{1}, m_{2}\right] * * a^{(i+1)}\left[m_{1}, m_{2}\right]-b^{(i+1)}\left[m_{1}, m_{2}\right] * * h^{(i)}\left[m_{1}, m_{2}\right]$

with $r^{(i)}\left[m_{1}, m_{2}\right]$ defined by:

$$
r^{(i)}\left[m_{1}, m_{2}\right]=r\left[m_{1}, m_{2}\right] * * h^{(i)}\left[m_{1}, m_{2}\right]
$$

with $(* *)$ representing the $2 \mathrm{D}$ convolution operation. For the $(i+1)$-th iteration, $a^{(i+1)}\left[m_{1}, m_{2}\right]$ and $b^{(i+1)}\left[m_{1}, m_{2}\right]$ in (7.12) are chosen to minimise $\left\|e^{(i+1)}\left[m_{1}, m_{2}\right]\right\|_{2}$ for given $r^{(i)}\left[m_{1}, m_{2}\right]$ and $h^{(i)}\left[m_{1}, m_{2}\right]$. Consequently, following LS formulation of (7.12) is proposed:

$$
\mathbf{e}^{(i+1)}=\left[\begin{array}{ll}
\mathbf{R}^{(i)} & \mathbf{H}^{(i)}
\end{array}\right]\left[\begin{array}{c}
\mathbf{a}^{(i+1)} \\
-\mathbf{b}^{(i+1)}
\end{array}\right]
$$

with

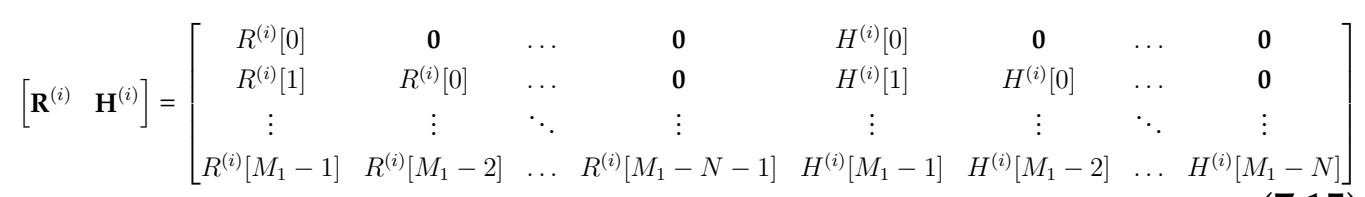

such that

$$
R^{(i)}[k]=\left[\begin{array}{cccc}
r^{(i)}[k, 0] & 0 & \ldots & 0 \\
r^{(i)}[k, 1] & r^{(i)}[k, 0] & \cdots & 0 \\
\vdots & \vdots & \ddots & \vdots \\
r^{(i)}\left[k, M_{2}-1\right] & r^{k,(i)}\left[M_{2}-2\right] & \cdots & r^{(i)}\left[k, M_{2}-N-1\right]
\end{array}\right] ; H^{(i)}[k]=\left[\begin{array}{cccc}
h^{(i)}[k, 0] & 0 & \ldots & 0 \\
h^{(i)}(k, 1] & h^{(i)}[k, 0] & \ldots & 0 \\
\vdots & \vdots & \vdots & \vdots \\
h^{(i)}\left[k, M_{2}-1\right] & h^{k(i)}\left[M_{2}-2\right] & \cdots & h^{(i)}\left[k, M_{2}-N\right]
\end{array}\right]
$$


and

$$
\mathbf{e}^{(i+1)}=\left[\begin{array}{c}
\boldsymbol{e}^{(i+1)}[0] \\
\boldsymbol{e}^{(i+1)}[1] \\
\vdots \\
\boldsymbol{e}^{(i+1)}\left[M_{1}-1\right]
\end{array}\right] ; \mathbf{a}^{(i+1)}=\left[\begin{array}{c}
\boldsymbol{a}^{(i+1)}[0] \\
\boldsymbol{a}^{(i+1)}[1] \\
\vdots \\
\boldsymbol{a}^{(i+1)}[N]
\end{array}\right] ; \mathbf{b}^{(i+1)}=\left[\begin{array}{c}
\boldsymbol{b}^{(i+1)}[0] \\
\boldsymbol{b}^{(i+1)}[1] \\
\vdots \\
\boldsymbol{b}^{(i+1)}[N-1]
\end{array}\right]
$$

such that

$$
\boldsymbol{e}^{(i+1)}[k]=\left[\begin{array}{c}
e^{(i+1)}[k, 0] \\
e^{(i+1)}[k, 1] \\
\vdots \\
e^{(i+1)}\left[k, M_{2}-1\right]
\end{array}\right] ; \boldsymbol{a}^{(i+1)}[k]=\left[\begin{array}{c}
a^{(i+1)}[k, 0] \\
a^{(i+1)}[k, 1] \\
\vdots \\
a^{(i+1)}[k, N]
\end{array}\right] ; \boldsymbol{b}^{(i+1)}[k]=\left[\begin{array}{c}
b^{(i+1)}[k, 0] \\
b^{(i+1)}[k, 1] \\
\vdots \\
b^{(i+1)}[k, N-1]
\end{array}\right]
$$

with $a^{(i)}[0,0]=1$. (7.14) leads to block LS Yule-Walker type equation [42] which can be solved for $\mathbf{a}^{(i+1)}$ and $\mathbf{b}^{(i+1)}$. The process in (7.12) is repeated until no further improvement in $e^{(i+1)}\left[m_{1}, m_{2}\right]$ is observed.

\subsection{Model order selection}

2D-SMA requires model order input $N$ in (7.8). The 2D Bayesian Information Criterion (BIC) is considered for this purpose [238]. The BIC is considered due to its superior performance when compared with alternatives, e.g., Akaike Information Criterion (AIC), Generalised Information Criterion (GIC), etc [202]. BIC adds an extra term to the LS cost function in (7.3).

$$
2 M_{1} M_{2} \log \left[\sum_{m_{1}=0}^{M_{1}-1} \sum_{m_{2}=0}^{M_{2}-1}\left|r\left(m_{1}, m_{2}\right)-\sum_{n=1}^{N} \zeta_{n} p_{n}^{m_{1}} q_{n}^{m_{2}}\right|^{2}\right]+8 N \log \left(M_{1} M_{2}\right)
$$

The extra term penalises higher order models if they do not yield a significant improvement in the residue. This prevents spurious estimates which do not actually contribute to the reconstruction of $r\left(m_{1}, m_{2}\right)$. Furthermore, this extra term does not incur a significant computational cost. 


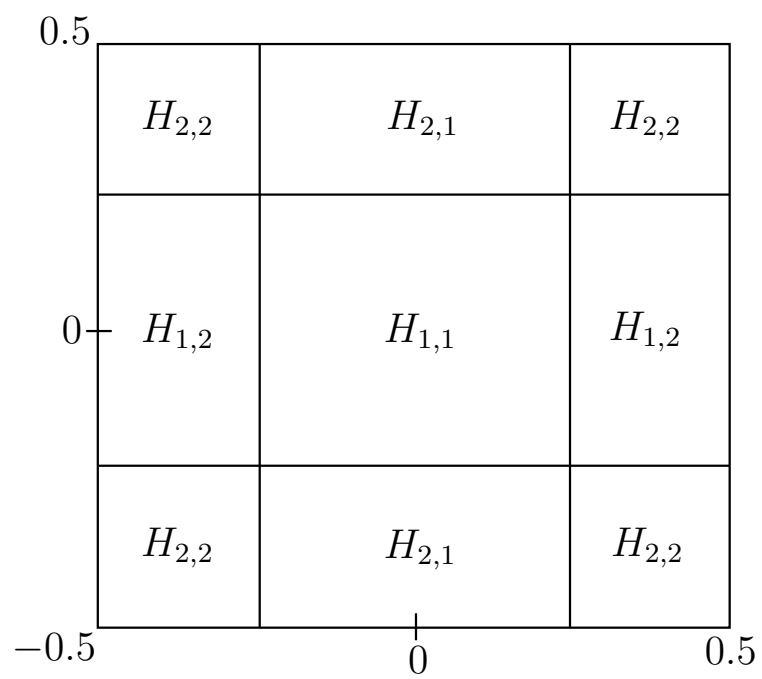

Figure 7.1: Schematic diagram of the 2D subband decomposition process with ideal frequency responses $\left\{H_{i, j}\left(f_{2}, f_{2}\right)\right\}_{i, j=1}^{2}$ [57].

\subsection{Adaptive subband decomposition}

Subband decomposition converts a fullband problem to numerous subband problems with the following immediate advantages in individual subbands: increase in Signal-to-Noise (SNR) ratio, increase in frequency resolution, decrease in signal length, and a decrease in computational complexity [212]. The above advantages help to improve the speed and accuracy of the quantitation algorithm in the individual subbands. For subband decomposition, we adopt a uniform, multi-step approach constituting successive filtering and decimation stages as depicted in Figure 7.1. Decomposition is obtained as follows [60]:

$$
r^{s, u}\left(m_{1}, m_{2}\right)=\sum_{n=1}^{N^{\prime}} \zeta_{n}^{\prime} p_{n}^{\prime m 1} q_{n}^{\prime m 2}+v^{s, u}\left(m_{1}, m_{2}\right)
$$

for $m_{1}=0,1, \ldots, M_{1}^{\prime}-1$ and $m_{2}=0,1, \ldots, M_{2}^{\prime}-1 . M_{1}^{\prime}$ and $M_{2}^{\prime}$ are the number of samples in the $(s, u)$-th subband with corresponding subband modes $N^{\prime} \leq N \cdot \zeta_{n^{\prime}}^{\prime} p_{n}^{\prime}$ and $q_{n}^{\prime}$ are subband counterparts of the fullband 
parameters $\zeta_{n}, p_{n}$ and $q_{n}$ respectively. Once the subband estimates $\hat{p}_{n}^{\prime}$ and $\hat{q}_{n}^{\prime}$ are obtained, they can be converted to fullband using:

$$
\begin{array}{ll}
\hat{p}_{n}=\left(\hat{p}_{n}^{\prime}\right)^{1 / 2 s} e^{j \pi \frac{2 u+1}{2^{3}+1}} & \hat{\omega}_{n}^{(1)} \in[0, \pi] \\
\hat{p}_{n}=\left(\hat{p}_{n}^{\prime}\right)^{1 / 2 s} e^{j \pi\left(\frac{2 u+1}{2^{s}+1}-1\right)} & \hat{\omega}_{n}^{(1)} \in[-\pi, 0]
\end{array}
$$

and

$$
\begin{array}{ll}
\hat{q}_{n}=\left(\hat{q}_{n}^{\prime}\right)^{1 / 2 s} e^{j \pi \frac{2 u+1}{2^{s}+1}} & \hat{\omega}_{n}^{(2)} \in[0, \pi] \\
\hat{q}_{n}=\left(\hat{q}_{n}^{\prime}\right)^{1 / 2 s} e^{j \pi\left(\frac{2 u+1}{2^{s}+1}-1\right)} & \hat{\omega}_{n}^{(2)} \in[-\pi, 0]
\end{array}
$$

Fullband estimates of $\hat{\zeta}_{n}^{\prime}$ are computed using (7.4). As the filters are not ideal, their responses will overlap as depicted in Figure 1. Consequently, a component may appear in more than one subband. The following criteria can be applied to uniquely determine the subband to which a mode belongs [212]:

$$
\max \left|R^{s, u}\left(e^{j \hat{\omega}_{n}^{\prime(1)}}, e^{j \hat{\omega}_{n}^{\prime(2)}}\right)\right|
$$

$\hat{\omega}_{n}^{\prime(1)}$ and $\hat{\omega}_{n}^{\prime(2)}$ are the frequencies chosen in the subband with the highest amplitude. Finally, the subband decomposition is made adaptive by employing the BIC-based order criterion in individual subbands [58].

\subsection{Algorithm}

The algorithm can be summarised as:

1. Data is checked for poles according to the 2D-BIC in (7.19).

2. Algorithm is halted if no poles are present.

3. Otherwise, poles are extracted using 2D-SMA, and the residual is subjected to 2D subband decomposition.

4. Steps from (1) to (3) are repeated until a global halt is reached. 
5. Estimated $\hat{p}_{n}^{\prime}$ and $\hat{q}_{n}^{\prime}$ are mapped to fullband values using (7.21) and (7.22).

6. $\zeta_{n}$ is computed using (7.4).

\subsection{Experimental results}

In this section, quantitation results obtained by the application of the proposed algorithm to experimental 2D-NMR data are presented. The The data was acquired from Biological Magnetic Resonance Data Bank, University of Wisconsin ${ }^{1}$. The recorded data was used as-is for algorithmic processing, i.e., without subjecting it to LP, apodization, zero-filling, phase and baseline correction or noise removal, etc. Fifth order coiflet filters were employed as 2D decimation filters due to their flat magnitude response, sharp cut-off and relatively small group delay [226]. The proposed algorithm was executed on an Intel $3.40 \mathrm{GHz}$ CPU. The algorithm converts the time-domain 2D-NMR data into a table of estimated 2D amplitudes, phases, frequencies and damping factors - magnitude of the components can be read directly from the table without requiring numerical integration of the peaks. These tables can be used for chemical fingerprinting, analysis of chemical concentrations in metabolomics, or monitoring of reactions in quality assurance and control [118]. But to visualise the results, we have converted the tables into conventional spectra.

Figure 7.2 presents the quantitation results obtained for the ${ }^{1} \mathrm{H}-{ }^{13} \mathrm{C}$ HSQC data of Nicotinamide Adenine Dinucleotide (NAD). The data was obtained by recording 256 complex data points in $t_{1}$ and 1024 complex data points in $t_{2}$ dimension. Stacked magnitude plots of spectra computed using FT and those reconstructed using (7.4) are displayed in Figures 7.2a and $7.2 \mathrm{~b}$ respectively.

Figure 7.2 reveals that much finer resolution is obtained in the spec-

\footnotetext{
${ }^{1}$ http://bmrb.wisc.edu/
} 


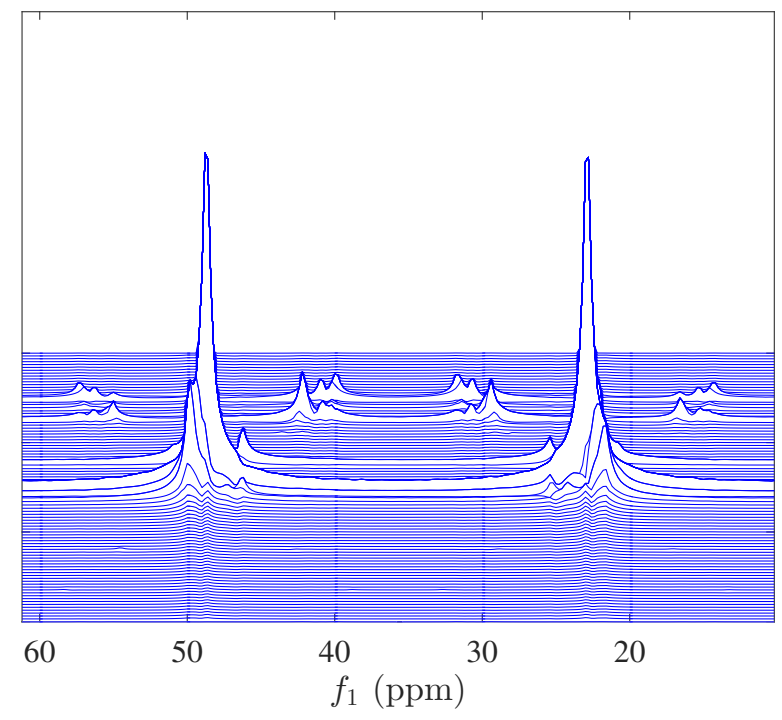

(a)

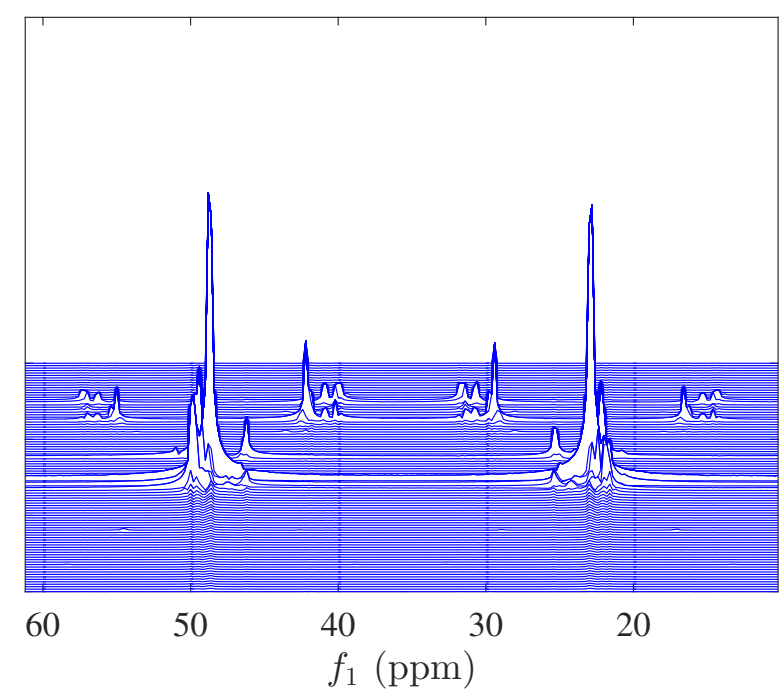

(b)

Figure 7.2: ${ }^{1} \mathrm{H}-{ }^{13} \mathrm{C}$ HSQC spectrum (Bruker DMX - 500Mhz) of nicotinamide adenine dinucleotide: (a)- stacked magnitude plot computed using FT, and (b)- reconstructed using (7.4). Processing time is 98.1 seconds. MSE is $5.8 \times 10^{-6}$. 
trum reconstructed using using (7.4) than the FT spectrum. FT was unable to resolve a number of spectral peaks and, for those resolved, was unable to predict the correct height. Similar observations were made by Belkic et al. [18] of FT when employed for quantitation of truncated 1D-NMR data.

This, once again, can be observed in Figure 7.3 for the ${ }^{1} \mathrm{H}-{ }^{1} \mathrm{H}$ COSY spectrum of HydroxyEthylPiperazine Ethane Sulfonic acid (HEPES). The spectrum reconstructed from the estimates generated by the proposed algorithm (Figure $7.3 b$ ) offers betters resolution than the one computed using FT (Figure 7.3a).

Figures 7.2 and 7.3 highlight the advantage of the proposed algorithm of obtaining high-resolution quantitation information from the truncated 2D-NMR data, keeping in view that it is almost invariably truncated in the indirect dimension [47]. Further, the NAD and HEPES data sets, of size $256 \times 1024$ each, were processed by the proposed algorithm in 98.1 and 81.3 seconds. This indicates that the processing speed of the proposed algorithm is not at all prohibitive, and highlights yet another advantage.

Finally, quantitation results for the data set originally employed in [119] are presented for further comparison. Figures 7.4a and 7.4b display the FT and reconstructed stacked magnitude for the zTOCSY spectrum of gibberellic acid. These show the improved spectral resolution achieved by the proposed algorithm for the given data set, i.e., the eight cross peaks are clearly resolved, which is also indicated by the achieved MSE of $3.1 \times 10^{-7}$. The CRAFT MSE was unspecified. The data set, of size $256 \times 1024$, was processed by the proposed algorithm in 73.7 seconds. The CRAFT processing time was 15 minutes. This once again shows the marked speed advantage of the proposed algorithm.

\subsection{Conclusion}

In this chapter, a 2D subband Steiglitz McBride algorithm - based on the 2D subband decomposition, the 2D Bayesian Information Criterion and 


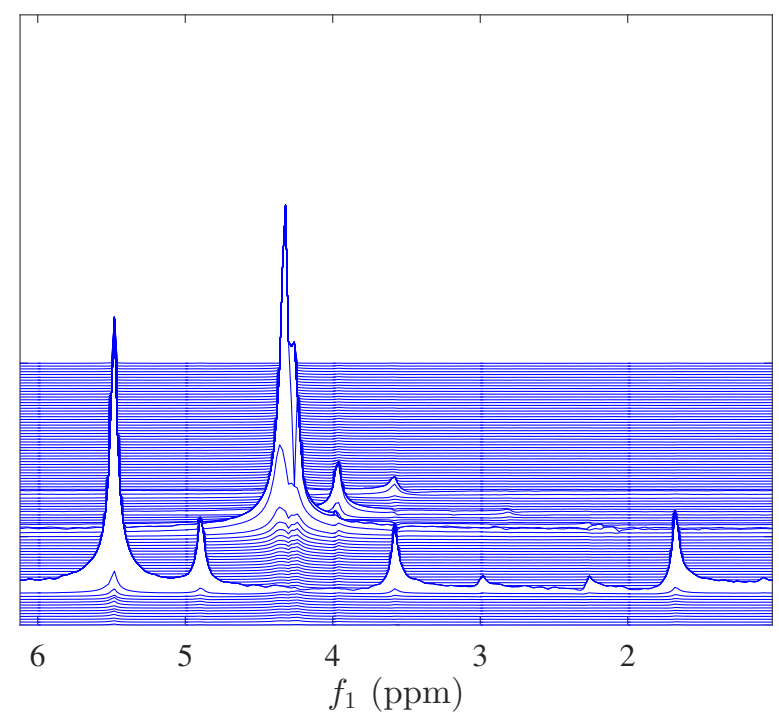

(a)

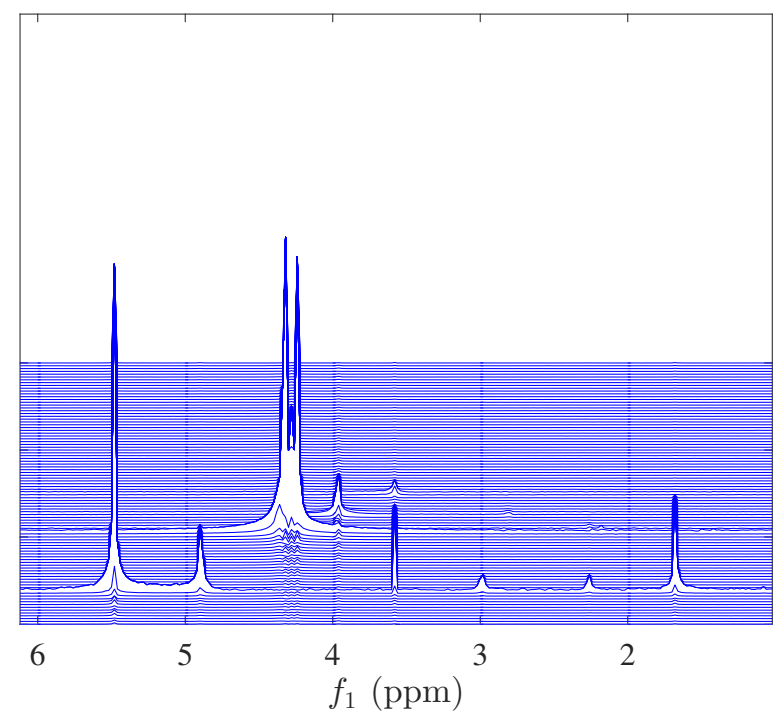

(b)

Figure 7.3: ${ }^{1} \mathrm{H}-{ }^{1} \mathrm{H}$ COSY spectrum (Bruker DMX - 500Mhz) of HEPES: (a)stacked magnitude plot computed using FT, and (b)- reconstructed using (7.4). Processing time is 81.3 seconds. MSE is $1.2 \times 10^{-6}$. 
a new formulation of the 2D Steiglitz McBride algorithm - was reported for rapid, high-resolution and automatic quantitation of 2D-NMR data. The algorithm is able to process the 2D-NMR data without requiring any previous knowledge or preprocessing, i.e., linear prediction, zero-filling or apodization, etc. The efficacy of the proposed algorithm was verified by its application to experimental 2D-NMR data. The algorithm outperformed the state-of-the-art in this area in terms of speed, resolution and aprioriinformation-free operation. 


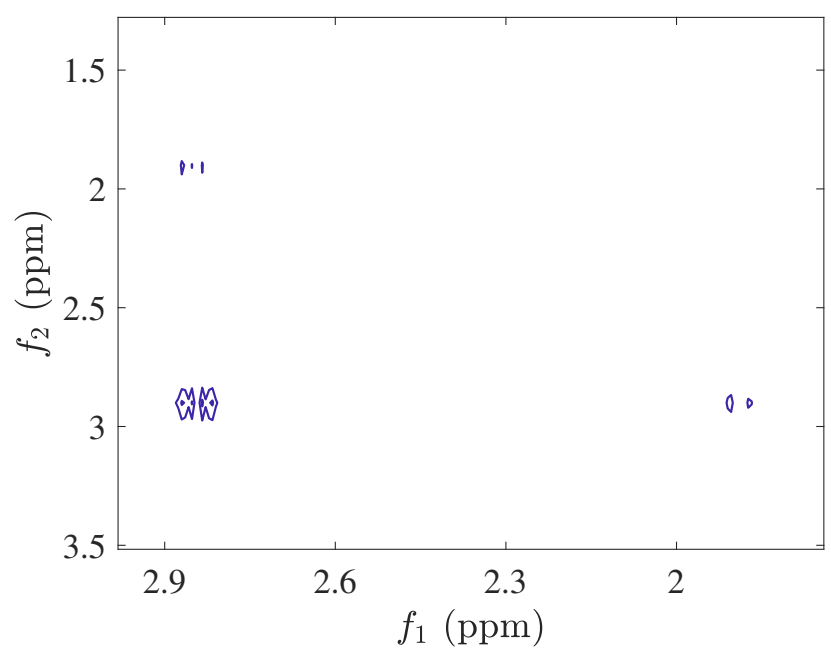

(a)

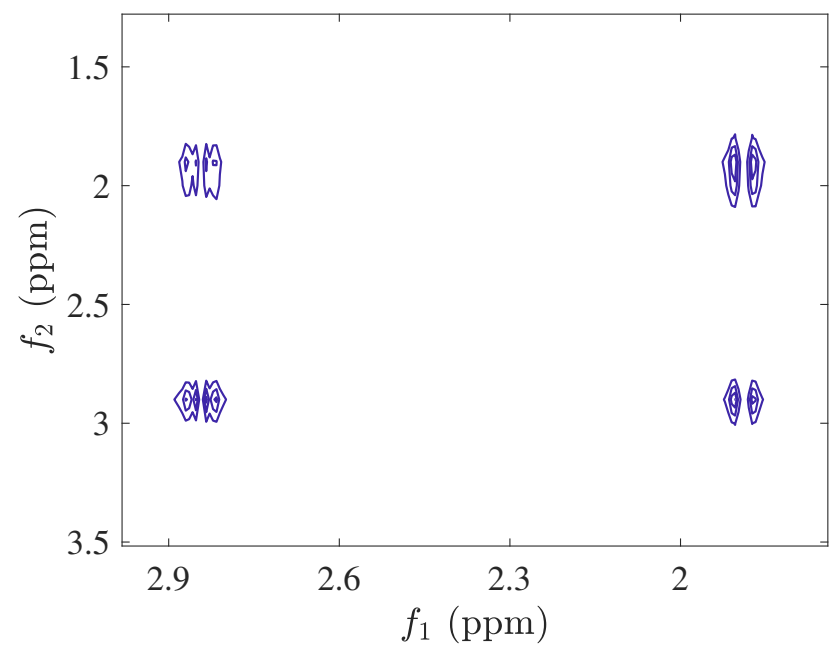

(b)

Figure 7.4: zTOCSY spectrum (Bruker DMX - 500Mhz) of gibberellic acid: (a)-computed using FT, and (b)- reconstructed using (7.4). Processing time is 73.7 seconds. MSE is $3.1 \times 10^{-7}$. 


\section{Chapter 8}

\section{Conclusions and future work}

This thesis reported research conducted to tackle the theoretical as well as practical problems encountered in parametric processing of NMR signals, firstly for 1D NMR signals and the later for 2D NMR signals. The contributions of this thesis can be grouped into following three categories:

- Analytical evaluation of the CRLB on unbiased parameter estimates of the damped cisoid model employed for representing the FID data in 1D-NMR,

- Development of a high-resolution, high-speed and aprioriinformation-free algorithm for parametric quantitation of 1D-NMR data, and

- Development of a similar algorithm, i.e., with similar quantitation properties, for parametric processing of two-dimensional 2D-NMR data.

\subsection{Conclusions}

Detailed conclusions of each category are outlined below. 


\subsubsection{Analytical evaluation of the CRLB}

For the CRLB, Chapter 3 reported analytic expressions on the variance of unbiased parameter estimates of damping factors, frequencies and complex amplitudes of an arbitrary number of damped cisoids embedded in AWGN - model employed in 1D-NMR data to represent the FID signal. In addition to the CRLB, analytic expressions for the determinant and the condition number of the associated Fisher Information Matrix (FIM) were also reported. Further results, in similar order, were reported for two special cases of the damped cisoid model: the Magnetic Resonance Relaxometry model and the amplitude-only model (employed in quantitative NMR - qNMR). Some auxiliary results for the above mentioned models were also presented, i.e., on the multiplicity of the eigenvalues, their separation, and the factorisation of the characteristic polynomial associated with their respective FIMs. The application of reported theoretical results to experimental data successfully accounted for various physical and chemical phenomena observed in MRS and quantified their impact on the accuracy of an unbiased estimator as a function of both model and experimental parameters.

\subsubsection{Quantitation algorithm for 1D-NMR data}

For rapid, high-resolution and apriori-information-free quantitation of 1D-NMR data, a sub-band Steiglitz-McBride algorithm - based on the Steiglitz McBride algorithm, the subband decomposition and the Bayesian Information Criterion - was reported in Chapter 5. The developed algorithm converts the time-domain FID data into a table of estimated amplitudes, phases, frequencies and damping factors. It does so without requiring any previous knowledge or preprocessing, i.e., base-line or phase correction, apodization, noise removal, etc. Application of the developed algorithm on experimental proton 1D-NMR data verified its superior performance in resolving dense, overlapped peaks with high dynamic range in a highly 
time-efficient manner when compared to the state-of-the-art in this area.

\subsubsection{Quantitation algorithm for 2D-NMR data}

For rapid, high-resolution and apriori-information-free quantitation of 2D-NMR data, a 2D sub-band Steiglitz-McBride algorithm - based on the 2D subband decomposition, the 2D Bayesian Information Criterion and a new formulation of the 2D Steiglitz McBride algorithm - was reported in Chapter 7. The algorithm converts the time-domain 2D NMR data into a table of 2D amplitudes, phases, frequencies and damping factors, without requiring any previous knowledge or preprocessing, i.e., linear prediction, zero-filling, apodization, etc., and is directly applicable to time-domain data from a variety of homonuclear, heteronuclear and spatial 2D NMR experiments. This was verified and by its application to data from homoand heteronuclear 2D NMR experiments. The algorithm yielded excellent quantitation results in terms of resolution, speed and automation, and considerably outperformed the state-of-the-art on all three fronts.

\subsection{Future work}

Following potential research directions are suggested by the results achieved in this thesis work:

- The first possibility concerns the work carried out in Chapter 3 regarding the derivation of the CRLB for parameter estimates of an arbitrary number of damped cisoids embedded in AWGN, i.e., the model representing the 1D-NMR data. This work may be extended to derive the CRLB for the 2D damped cisoid model representing the 2D-NMR data. Theoretical insights gained from this extension can be employed to further improve the performance of the developed 2D-NMR quantitation algorithm (Chapter 7) in particular, and any 2D-NMR quantitation algorithm in general, 
- A second possibility would be to extend the 2D quantitation algorithm (Chapter 7) for rapid, high-resolution and apriori-informationfree quantitation of 3D-NMR data and, in general, ND-NMR data,

- A third possibility would be to embed in the developed 1D and 2D algorithms, the ability to incorporate previous knowledge about the chemical shifts - as in certain applications, one or more of the underlying frequencies in NMR signal are known, e.g., in NMR-based metabolomics [113].

- A fourth possibility would be to incorporate in the developed 1D and 2D quantitation algorithms, the ability to process non-uniformly sampled data, both on a deterministic grid or an altogether random grid. Non-uniform sampling is becoming increasingly important in the acquisition and processing of higher dimensional NMR signals [51].

- Alluding to the fourth possibility, an alternative approach to the processing of non-uniform sampling can be adopted by deriving the analytical CRLB results (analogous to those in Chapter 3) for the nonuniform sampling case. Analytical CRLB results may lead to explicit insights into the design of non-uniform sampling schemes which can preserve the spectral content while keeping the number of sampling points minimum.

- A sixth possibility would be to explore, and possibly mitigate, the impact of the magnetic inhomogeneities which give rise to the nonLorentzian peaks [32]. For example, we know from our discussion in Chapter 6 that these magnetic inhomogeneities arise due to the nuclei that are either bonded or are in close spatial proximity to each other. The magnetic effects of these nuclei are neutralised by the application of double and triple pulse sequences in COSY and NOESY respectively. Once these pulse have been applied, the resulting FIDs 
can be acquired [74] and the impact of these pulses on the peak shape can be explored (quantitatively) using the 1D-NMR quantitation algorithm reported in Chapter 5. If the procedure is successful in the restoration of the Lorentzian peak shapes, the acquisition time for the 2D-NMR experiments, which can exceed an hour, can be reduced considerably. It is important to note that conventional 2D-NMR experiments, like COSY and NOESY, acquire the whole set of 2D-FIDs to improve the resolution of overlapping peaks. By contrast, the objective in this scenario will be to acquire and analyse the FIDs generated after the application of initial mitigating pulses, so that the impact on these pulses on peak shapes can be observed explicitly.

- A seventh possibility, connected to the eighth, focuses on the impact of magnetic inhomogeneities on the performance of the quantitaion algorithms only. Since a damped ciosid term in the damped cisoid model represents a Lorentzian peak shape in the frequency domain, a Gaussian or a Viogt peak shape arising due to the magnetic inhomogeneities may be represented by the quantitation method as a superposition of individual Lorentzian peaks [94] - a single peak appearing as a sum of numerous individual peaks. This situation may be compounded after subband decomposition in which one peak, after the decomposition, may appear in more than one subbands. A group of such related peaks, treated as a single unit (singlet), can reduce the number of poles and, in turn, the number of parameters to be estimated in the model. Furthermore, the possibility of multiplets can also be explored which can further reduce the redundant estimates.

- An eighth possibility concerns, in addition to the multiplets, other artefacts present in the FID signal that are ignored by the timedomain model. For example, the time-domain model implies that the phases of the individual peaks can be different, which is not nec- 
essarily true. If the peaks are correlated (i.e., they have the same phase), it will reduce the number of unknowns to be estimated in the model [147], a possibility which can be taken into account. Another possibility that needs to be addressed is the impact of the constant offsets, in damping factors and frequencies, caused by imperfections in the amplifier. These offsets, if modelled, can reduce the number of artefacts in the model. Furthermore, base-line distortion, which severely hampers the performance of many excellent quantitation methods discussed in Chapter 04, could also be modelled in the time-domain model of the FID signal.

- Finally, yet another possibility concerns the reduction of acquisition time for 2D-NMR experiments, and originates from the observation that the indirect dimension in such experiments can be only partially occupied, i.e., chemically meaningful peaks may appear only at few locations, leaving rest of the axis largely unoccupied [170]. Compressed sensing framework, for sparse spectral reconstruction in the indirect dimension, can be integrated into the 2D-NMR quantitation algorithm developed in Chapter 07. 


\section{Bibliography}

[1] Abed-Meraim, K., HuA, Y., And Belouchrani, A. Second-order near-field source localization: algorithm and performance analysis. In Signals, Systems and Computers, 1996. Conference Record of the Thirtieth Asilomar Conference on (1996), vol. 1, IEEE, pp. 723-727.

[2] ABEL, N. H. Mmoire sur les equations algbriques, o l'on dmontre l'impossibilit de la rsolution de l'quation gnrale du cinquime degr, vol. 1 of Cambridge Library Collection - Mathematics. Cambridge University Press, 2012, pp. 28-33.

[3] Aboutanios, E., Thomas, D. S., Hook, J. M., And Cobas, C. LocMAP: A new localization method for the parametric processing of high resolution nmr data. Journal of Magnetic Resonance 282 (2017), $62-70$.

[4] Abramowitz, M., AND Stegun, I. A. Handbook of mathematical functions: with formulas, graphs, and mathematical tables, vol. 55. Courier Corporation, 1964.

[5] Ackermann, S. M., Dolsophon, K., Monakhova, Y. B., Kuballa, T., Reusch, H., ThongPanchang, T., Bunzel, M., AND LACHENMEIER, D. W. Automated multicomponent analysis of soft drinks using ${ }_{1} \mathrm{D}^{1} \mathrm{H}$ and ${ }_{2} \mathrm{D}^{1} \mathrm{H}-{ }^{1} \mathrm{H}$ J-resolved NMR spectroscopy. Food Analytical Methods 10, 3 (2017), 827-836. 
[6] Ala-Korpela, M., Korhonen, A., Keisala, J., HÖrkKÖ, S., KORPI, P., INGMAN, L., JOKISAARI, J., SAVOlAINEN, M., AND KESÄNIEMI, Y. ${ }^{1} \mathrm{H}$ NMR-based absolute quantitation of human lipoproteins and their lipid contents directly from plasma. Journal of lipid research 35, 12 (1994), 2292-2304.

[7] Alexanderian, A., Petra, N., Stadler, G., and Ghattas, O. A-optimal design of experiments for infinite-dimensional Bayesian linear inverse problems with regularized $\ell_{0}$-sparsification. SIAM Journal on Scientific Computing 36, 5 (2014), A2122-A2148.

[8] Andrews, D. L., Belton, P., Creaser, C., Cudby, M., KetTle, S., McCoustra, M., Mellon, F., Powell, D., Sheppard, N., SodeAu, J., ET AL. Perspectives in modern chemical spectroscopy. Springer Science \& Business Media, 2012.

[9] Antoine, J.-P., CORON, A., AND Dereppe, J.-M. Water peak suppression: time-frequency vs. time-scale approach. Journal of Magnetic Resonance 144, 2 (2000), 189-194.

[10] AtKinson, A. C., And Fedorov, V. V. Optimal design: Experiments for discriminating between several models. Biometrika 62, 2 (1975), 289-303.

[11] Aue, W., Bartholdi, E., And ERnst, R. R. Two-dimensional spectroscopy. application to nuclear magnetic resonance. The Journal of Chemical Physics 64, 5 (1976), 2229-2246.

[12] BADAWI, H. M., FÖRner, W., AND Ali, S. A. A study of the experimental and theoretical infrared, raman, ${ }^{1} \mathrm{H}$ and ${ }^{13} \mathrm{C}$ NMR spectra of the biochemicals valeric and valproic acids. Journal of Molecular Structure 1075 (2014), 494-503. 
[13] BADEAU, R., David, B., AND Richard, G. Cramer-Rao bounds for multiple poles and coefficients of quasi-polynomials in colored noise. IEEE Transactions on Signal Processing 56, 8 (2008), 3458-3467.

[14] Barkhuijsen, H., De Beer, R., Bovee, W., Creyghton, J., And VAN ORMONDT, D. Application of linear prediction and singular value decomposition (LPSVD) to determine NMR frequencies and intensities from the FID. Magnetic resonance in medicine 2, 1 (1985), 86-89.

[15] Bartha, R., Drost, D., AND Williamson, P. Factors affecting the quantification of short echo in-vivo ${ }^{1} \mathrm{H}$ MR spectra: prior knowledge, peak elimination, and filtering. NMR in biomedicine 12, 4 (1999), 205-216.

[16] BECKER, E. D. A brief history of nuclear magnetic resonance. Analytical chemistry 65, 6 (1993), 295A-302A.

[17] Beckonert, O., Keun, H. C., Ebbels, T. M., Bundy, J., Holmes, E., Lindon, J. C., AND Nicholson, J. K. Metabolic profiling, metabolomic and metabonomic procedures for NMR spectroscopy of urine, plasma, serum and tissue extracts. Nature protocols 2, 11 (2007), 2692-2703.

[18] BelKić, D., AND BelKić, K. Fast Padé transform in the theory of resonances: exact solution of the harmonic inversion problem. Journal of Physics B: Atomic, Molecular and Optical Physics 44, 12 (2011), 125002.

[19] Bertelli, D., Lolli, M., Papotti, G., Bortolotti, L., Serra, G., AND PLESSI, M. Detection of honey adulteration by sugar syrups using one-dimensional and two-dimensional high-resolution nuclear magnetic resonance. Journal of agricultural and food chemistry 58,15 (2010), 8495-8501. 
[20] BEST, G. Notes on the Graeffe method of root squaring. The American Mathematical Monthly 56, 2 (1949), 91-94.

[21] Bi, Z., Bruner, A. P., Li, J., Scott, K. N., LiU, Z.-S., STOPKA, C. B., KIM, H.-W., AND WILSON, D. C. Spectral fitting of nmr spectra using an alternating optimization method with a priori knowledge. Journal of Magnetic Resonance 140, 1 (1999), 108-119.

[22] BitTL, J. A., AND INGWALL, J. S. Reaction rates of creatine kinase and ATP synthesis in the isolated rat heart. a ${ }^{31} \mathrm{P}$ NMR magnetization transfer study. Journal of Biological Chemistry 260, 6 (1985), 35123517.

[23] BJÖrK, M., Zachariah, D., Kullberg, J., And StoicA, P. A multicomponent $T_{2}$ relaxometry algorithm for myelin water imaging of the brain. Magnetic resonance in medicine 75, 1 (2016), 390-402.

[24] Blanazs, A., Bristow, T. W., COOMbes, S. R., CORRY, T., NunN, M., AND RAY, A. D. Coupling and optimisation of online nuclear magnetic resonance spectroscopy and mass spectrometry for process monitoring to cover the broad range of process concentration. Magnetic Resonance in Chemistry 55, 4 (2017), 274-282.

[25] Bodenhausen, G. Direct determination of rate constants of slow dynamic processes by two-dimensional" accordion" spectroscopy in nuclear magnetic resonance. Journal of the American Chemical Society 104, 5 (1982), 1304-1309.

[26] Boogaart, V., Den, A., Howe, F., Rodrigues, L., Stubbs, M., AND GRIFFITHS, J. In vivo ${ }^{31} \mathrm{P}$ MRS: absolute concentrations, signalto-noise and prior knowledge. NMR in biomedicine 8, 2 (1995), 87-93.

[27] Bouhrara, M., AND Spencer, R. G. Fisher information and cramér-rao lower bound for experimental design in parallel imaging. Magnetic Resonance in Medicine 79, 6 (2018), 3249-3255. 
[28] Bracewell, R. The Fourier transform and its applications. New York 5 (1965).

[29] Breit, G., ANd Rabi, I. Measurement of nuclear spin. Physical Review 38, 11 (1931), 2082.

[30] BResler, Y., AND MAcovsKI, A. Exact maximum likelihood parameter estimation of superimposed exponential signals in noise. IEEE Transactions on Acoustics, Speech, and Signal Processing 34, 5 (1986), 1081-1089.

[31] BRETTHORST, G. L. How accurately can parameters from exponential models be estimated? a Bayesian view. Concepts in Magnetic Resonance Part A 27, 2 (2005), 73-83.

[32] BRETTHORST, G. L. Bayesian spectrum analysis and parameter estimation, vol. 48. Springer Science \& Business Media, 2013.

[33] Brown, G. G., Dian, B. C., Douglass, K. O., Geyer, S. M., Shipman, S. T., AND PATE, B. H. A broadband Fourier transform microwave spectrometer based on chirped pulse excitation. Review of Scientific Instruments 79, 5 (2008), 053103.

[34] Callaghan, P. T. Translational dynamics and magnetic resonance: principles of pulsed gradient spin echo NMR. Oxford University Press, 2011.

[35] Campbell, G. C., Crosby, R. C., and Haw, J. F. ${ }^{13}$ C chemical shifts which obey the curie law in CP/MAS NMR spectra. the first CP/MAS NMR chemical-shift thermometer. Journal of Magnetic Resonance (1969) 69, 1 (1986), 191-195.

[36] Carbajo, R. J., AND Neira, J. L. NMR for Chemists and Biologists. Springer, 2013. 
[37] Cavassila, S., Deval, S., Huegen, C., Van Ormondt, D., And GRAVERON-DEMILly, D. Cramer-Rao bound expressions for parametric estimation of overlapping peaks: influence of prior knowledge. Journal of Magnetic Resonance 143, 2 (2000), 311-320.

[38] CeliK, H. Development of New Signal Processing and Mixture Separation Techniques for Nuclear Magnetic Resonance Spectroscopy Applications. University of California, Irvine, 2011.

[39] Chen, H., VAn Huffel, S., VAn ORmondt, D., AND DE BeER, R. Parameter estimation with prior knowledge of known signal poles for the quantification of NMR spectroscopy data in the time domain. Journal of Magnetic Resonance, Series A 119, 2 (1996), 225-234.

[40] Chen, H., Vanhuffel, S., Decanniere, C., And Vanhecke, P. A signal-enhancement algorithm for the quantification of NMR data in the time domain. Journal of Magnetic Resonance, Series A 109, 1 (1994), 46-55.

[41] Chen, L., Weng, Z., Goh, L., And Garland, M. An efficient algorithm for automatic phase correction of NMR spectra based on entropy minimization. Journal of Magnetic Resonance 158, 1 (2002), 164-168.

[42] ChOI, B., AND Politis, D. N. Modeling 2-D AR processes with various regions of support. IEEE Transactions on Signal Processing 55, 5 (May 2007), 1696-1707.

[43] Chuang, C., AND Moffatt, D. Natural resonances of radar targets via Prony's method and target discrimination. IEEE Transactions on Aerospace and Electronic Systems, 5 (1976), 583-589.

[44] ClARK, M. P., AND SCHARF, L. L. Two-dimensional modal analysis based on maximum likelihood. IEEE Transactions on Signal Processing 42, 6 (1994), 1443-1452. 
[45] Comisarow, M. B., AND Marshall, A. G. Fourier transform ion cyclotron resonance spectroscopy. Chemical Physics Letters 25, 2 (1974), 282-283.

[46] Croux, C., And Haesbroeck, G. Influence function and efficiency of the minimum covariance determinant scatter matrix estimator. Journal of Multivariate Analysis 71, 2 (1999), 161-190.

[47] Dashti, H. T. Computational aspects of protein NMR. PhD thesis, The University of Wisconsin-Madison, 2015.

[48] De GraAf, A., AND Bovee, W. Improved quantification of in vivo ${ }^{1} \mathrm{H}$ NMR spectra by optimization of signal acquisition and processing and by incorporation of prior knowledge into the spectral fitting. Magnetic Resonance in Medicine 15, 2 (1990), 305-319.

[49] De GraAf, A., VAn DijK, J., AND BoÉE, W. Quality: quantification improvement by converting lineshapes to the Lorentzian type. Magnetic resonance in medicine 13, 3 (1990), 343-357.

[50] De Groen, P., And De Moor, B. The fit of a sum of exponentials to noisy data. Journal of Computational and Applied Mathematics 20 (1987), 175-187.

[51] Delaglio, F., Walker, G. S., Farley, K. A., Sharma, R., Hoch, J. C., Arbogast, L. W., Brinson, R. G., AND Marino, J. P. Nonuniform sampling for all: More NMR spectral quality, less measurement time. American pharmaceutical review 20, 4 (2017).

[52] Dellalunga, G., Pogni, R., And Basosi, R. A simple method for baseline correction in EPR spectroscopy. Journal of Magnetic Resonance: Series A 108, 1 (1994), 65-70.

[53] Derrien, O., Badeau, R., And Richard, G. Parametric audio coding with exponentially damped sinusoids. IEEE Transactions on Audio, Speech, and Language Processing 21, 7 (2013), 1489-1501. 
[54] Dimarogonas, A. D., Paipetis, S. A., And Chondros, T. G. Approximate evaluation of eigenfrequencies. In Analytical Methods in Rotor Dynamics. Springer, 2013, pp. 1-23.

[55] Diop, A., Kolbel, W., Michel, D., Briguet, A., AND GRAVERONDEMILLY, D. Full automation of quantitation of in vivo NMR by LPSVD (CR) and EPLPSVD. Journal of Magnetic Resonance, Series B 103, 3 (1994), 217-221.

[56] Diop, A., Zaimwadghiri, Y., Briguet, A., AND GraverondeMILLY, D. Improvements of quantitation by using the Cadzow enhancement procedure prior to any linear-prediction methods. Journal of Magnetic Resonance, Series B 105, 1 (1994), 17-24.

[57] Djermoune, E.-H., BRIE, D., AND TOMCZAK, M. A subband algorithm for estimating the parameters of two-dimensional exponential signals. In EUSIPCO (2009), pp. 1032-1036.

[58] Djermoune, E.-H., AND TOMCZAK, M. An adapted filter bank for frequency estimation. In Signal Processing Conference, 2004 12th European (2004), IEEE, pp. 2171-2174.

[59] DJermoune, E.-H., TOMCZAK, M., AND BRIE, D. NMR data analysis: A time-domain parametric approach using adaptive subband decomposition. Oil \& Gas Science and Technology-Revue dIFP Energies nouvelles 69, 2 (2014), 229-244.

[60] Djermoune, E.-H., Tomczak, M., And Mutzenhardt, P. A new adaptive subband decomposition approach for automatic analysis of NMR data. Journal of Magnetic Resonance 169, 1 (2004), 73-84.

[61] DogAncAY, K. Online optimization of receiver trajectories for scanbased emitter localization. IEEE Transactions on Aerospace and Electronic Systems 43, 3 (2007). 
[62] DogancaY, K. UAV path planning for passive emitter localization. IEEE Transactions on Aerospace and Electronic systems 48, 2 (2012), 1150-1166.

[63] Doolette, A. L., AND SMERnIK, R. J. Quantitative analysis of ${ }^{31} \mathrm{P}$ NMR spectra of soil extracts-dealing with overlap of broad and sharp signals. Magnetic Resonance in Chemistry 53, 9 (2015), 679-685.

[64] Doorsamy, W., Abdallh, A. A.-E., Cronje, W. A., AND Dupré, L. An experimental design for static eccentricity detection in synchronous machines using a Cramer-Rao lower bound technique. IEEE Transactions on Energy Conversion 30, 1 (2015), 254-261.

[65] El Korso, M. N., Boyer, R., Renaux, A., And Marcos, S. Conditional and unconditional Cramer-Rao bounds for near-field source localization. IEEE Transactions on Signal Processing 58, 5 (2010), 29012907.

[66] El Korso, M. N., and Pesavento, M. Performance analysis for near field source localization. In 2012 IEEE 7th Sensor Array and Multichannel Signal Processing Workshop (SAM) (2012), IEEE, pp. 197-200.

[67] Engelsen, S. B., AND VAN DEN BerG, F. W. Quantitative analysis of time domain NMR relaxation data. In Modern Magnetic Resonance. Springer, 2017, pp. 1-19.

[68] ERnSt, R., AND ANDERSON, W. Application of Fourier transform spectroscopy to magnetic resonance. Review of Scientific Instruments 37, 1 (1966), 93-102.

[69] ERnSt, R., AND PRimas, H. High resolution NMRinstrumentation: recent advances and prospects. Discussions of the Faraday Society 34 (1962), 43-51.

[70] Faddeev, D. K., AND Faddeva, V. N. Computational Methods of Linear Algebra. 1963. 
[71] Farmakis, I., And Moskowitz, M. A Graduate Course in Algebra: (In 2 Volumes). World Scientific Publishing Company, 2017.

[72] FenG, Z.-K., NiU, W.-J., ChenG, C.-T., AND LiaO, S.-L. Hydropower system operation optimization by discrete differential dynamic programming based on orthogonal experiment design. Energy 126 (2017), 720-732.

[73] FERENTZ, A. E., AND WAGNer, G. NMR spectroscopy: a multifaceted approach to macromolecular structure. Quarterly reviews of biophysics 33, 1 (2000), 29-65.

[74] Field, L., Magill, A. M., AND LI, H. L. Organic Structures from 2D NMR Spectra. John Wiley \& Sons, 2015.

[75] FRAME, J. A simple recursion formula for inverting a matrix. In Bulletin of the American Mathematical Society (1949), vol. 55, pp. 10451045.

[76] FREEMAN, R., AND WHIFFEN, D. Determination of the relative signs of proton spin coupling constants by double irradiation. Molecular Physics 4, 4 (1961), 321-325.

[77] FuKUSHIMA, E. Nuclear magnetic resonance as a tool to study flow. Annual review of fluid mechanics 31, 1 (1999), 95-123.

[78] Gesmar, H., AND Led, J. J. Spectral estimation of complex timedomain NMR signals by linear prediction. Journal of Magnetic Resonance (1969) 76, 1 (1988), 183-192.

[79] Gill, P. E., AND Murray, W. Algorithms for the solution of the nonlinear least-squares problem. SIAM Journal on Numerical Analysis 15, 5 (1978), 977-992.

[80] GJerde, M. I., Nerdal, W., AND Høiland, H. A NOESY NMR study of the interaction between sodium dodecyl sulfate and poly 
(ethylene oxide). Journal of colloid and interface science 183, 1 (1996), 285-288.

[81] Goffe, W. L., Ferrier, G. D., AND Rogers, J. Global optimization of statistical functions with simulated annealing. Journal of econometrics 60, 1-2 (1994), 65-99.

[82] Golub, G., And Pereyra, V. Separable nonlinear least squares: the variable projection method and its applications. Inverse problems 19, 2 (2003), R1.

[83] Golub, G. H., AND VAN LOAN, C. Total least squares. In Smoothing Techniques for Curve Estimation. Springer, 1979, pp. 69-76.

[84] Grabow, J.-U., Palmer, E. S., McCarthy, M. C., And ThadDEUS, P. Supersonic-jet cryogenic-resonator coaxially oriented beam-resonator arrangement Fourier transform microwave spectrometer. Review of Scientific Instruments 76, 9 (2005), 093106.

[85] GRAHAM, S. J., AND BRONSKILL, M. J. MR measurement of relative water content and multicomponent $T_{2}$ relaxation in human breast. Magnetic Resonance in Medicine 35, 5 (1996), 706-715.

[86] Graham, S. J., Stanchev, P. L., And Bronskill, M. J. Criteria for analysis of multicomponent tissue $T_{2}$ relaxation data. Magnetic Resonance in Medicine 35, 3 (1996), 370-378.

[87] Gudmundson, E., Stoica, P., Li, J., Jakobsson, A., Rowe, M. D., SMith, J. A., AND LiNG, J. Spectral estimation of irregularly sampled exponentially decaying signals with applications to RF spectroscopy. Journal of Magnetic Resonance 203, 1 (2010), 167-176.

[88] GÜNTHER, H. NMR spectroscopy: basic principles, concepts and applications in chemistry. John Wiley \& Sons, 2013. 
[89] Guthausen, G. Analysis of food and emulsions. TrAC Trends in Analytical Chemistry 83 (2016), 103-106.

[90] Hansen, A. L., Li, D., WANG, C., AND BRÜSChweIler, R. Absolute minimal sampling of homonuclear 2D NMR TOCSY spectra for high-throughput applications of complex mixtures. Angewandte Chemie 129, 28 (2017), 8261-8264.

[91] HAnson, J. Organic chemistry; a mechanistic approach. Science Progress 98, 2 (2015), 209-209.

[92] Hartley, H. O. The modified Gauss-Newton method for the fitting of non-linear regression functions by least squares. Technometrics 3, 2 (1961), 269-280.

[93] Herbert, S., Hopgood, J. R., And Mulgrew, B. Optimality criteria for adaptive waveform design in MIMO radar systems. In Sensor Signal Processing for Defence Conference (SSPD), 2017 (2017), IEEE, pp. 1-5.

[94] Higinbotham, J., And Marshall, I. NMR lineshapes and lineshape fitting procedures.

[95] Hill, E., AND LandShOFF, R. The Dirac electron theory. Reviews of Modern Physics 10, 2 (1938), 87.

[96] Hiltunen, Y., KaArtinen, J., Pulkkinen, J., HäKkinen, A.-M., LundBOM, N., AND KAUPPINEN, R. A. Quantification of human brain metabolites from in vivo ${ }^{1} \mathrm{H}$ NMR magnitude spectra using automated artificial neural network analysis. Journal of Magnetic Resonance 154,1 (2002), 1-5.

[97] Hochwald, B., AND NehORAI, A. Concentrated Cramer-Rao bound expressions. IEEE transactions on information theory 40, 2 (1994), 363-371. 
[98] Hofmann, L., Slotboom, J., Boesch, C., And Kreis, R. Model fitting of ${ }^{1} \mathrm{H}$ MR spectra of the human brain: incorporation of short- $T_{1}$ components and evaluation of parameterized vs. nonparameterized models. In Proceedings of the 7th Annual Meeting of ISMRM, Philadelphia (1999), p. 586.

[99] HolzGrabe, U. Quantitative NMR spectroscopy in pharmaceutical applications. Progress in Nuclear Magnetic Resonance Spectroscopy 57, 2 (2010), 229-240.

[100] Holzgrabe, U. NMR spectroscopy in pharmaceutical analysis. Elsevier, 2017.

[101] Holzgrabe, U., WAWER, I., AND DieHL, B. NMR spectroscopy in drug development and analysis. John Wiley \& Sons, 2008.

[102] HU, Z., GAO, S., AND XIANG, B. Time-delayed behaviors of transient four-wave mixing signal intensity in inverted semiconductor with carrier-injection pumping. Optics Communications 359 (2016), 329-335.

[103] HUA, Y. Estimating two-dimensional frequencies by matrix enhancement and matrix pencil. IEEE Transactions on Signal Processing 40, 9 (1992), 2267-2280.

[104] HWANG, T.-L., AND SHAKA, A. Water suppression that works. excitation sculpting using arbitrary waveforms and pulsed field gradients. Journal of Magnetic Resonance: Series A 112 (1995), 275-275.

[105] Istratov, A. A., AND VyvenKo, O. F. Exponential analysis in physical phenomena. Review of Scientific Instruments 70, 2 (1999), 1233-1257.

[106] JaGANNATHAN, N. Quantitative estimate of in-vivo metabolites in breast and prostate tissues by MR spectroscopy. Medical Physics 36 (2009), 2768. 
[107] Jaravine, V., Ibraghimov, I., AND OReKhov, V. Y. Removal of a time barrier for high-resolution multidimensional NMR spectroscopy. Nature methods 3, 8 (2006), 605-607.

[108] JARDETZKY, O., AND JARDETZKY, C. D. An interpretation of the proton magnetic resonance spectrum of ribonuclease. Journal of the American Chemical Society 79, 19 (1957), 5322-5323.

[109] JIRU, F. Introduction to post-processing techniques. European journal of radiology 67,2 (2008), 202-217.

[110] Jones, J., Hodgkinson, P., Barker, A., And Hore, P. Optimal sampling strategies for the measurement of spin-spin relaxation times. Journal of Magnetic Resonance: Series B 113, 1 (1996), 25-34.

[111] KAMMER, D. C. Sensor placement for on-orbit modal identification and correlation of large space structures. Journal of Guidance, Control, and Dynamics 14, 2 (1991), 251-259.

[112] KAY, S. M. Fundamentals of statistical signal processing, volume I: estimation theory. Prentice Hall, 1993.

[113] KeUn, H. C., Ed. NMR-based Metabolomics. New Developments in NMR. The Royal Society of Chemistry, 2018.

[114] KÖLBEL, W., AND SCHÄFER, H. Improvement and automation of the LPSVD algorithm by continuous regularization of the singular values. Journal of Magnetic Resonance (1969) 100, 3 (1992), 598-603.

[115] Kopchenova, N., Kopcenova, N., And Maron, I. Computational mathematics. Mir Publishers Moscow, 1984.

[116] Korostynska, O., Mason, A., And Al-Shamma'A, A. Microwave sensors for the non-invasive monitoring of industrial and medical applications. Sensor Review 34, 2 (2014), 182-191. 
[117] KRANE, K. S., AND HALLIDAY, D. Introductory nuclear physics, vol. 465. Wiley New York, 1988.

[118] KRISHNAMURTHY, K. CRAFT (complete reduction to amplitude frequency table)-robust and time-efficient bayesian approach for quantitative mixture analysis by NMR. Magnetic Resonance in Chemistry 51, 12 (2013), 821-829.

[119] Krishnamurthy, K., Sefler, A. M., And Russell, D. J. Application of CRAFT in two-dimensional NMR data processing. Magnetic Resonance in Chemistry 55, 3 (2017), 224-232.

[120] Kuballa, T., Brunner, T. S., Thongpanchang, T., Walch, S. G., AND LACHENMEIER, D. W. Application of NMR for authentication of honey, beer and spices. Current Opinion in Food Science 19 (2018), 57-62.

[121] KUMAR, A., ERNST, R., AND WÜTHRICH, K. A two-dimensional nuclear overhauser enhancement (2D NOE) experiment for the elucidation of complete proton-proton cross-relaxation networks in biological macromolecules. Biochemical and biophysical research communications 95, 1 (1980), 1-6.

[122] Kumaresan, R., AND TufTS, D. Estimating the parameters of exponentially damped sinusoids and pole-zero modeling in noise. IEEE Transactions on Acoustics, Speech, and Signal Processing 30, 6 (1982), 833-840.

[123] KupČE, E., AND FREEMAN, R. SPEED: single-point evaluation of the evolution dimension. Magnetic Resonance in Chemistry 45, 9 (2007), 711-713.

[124] LAATIKAINEN, R. Automated analysis of NMR spectra. Journal of Magnetic Resonance (1969) 92, 1 (1991), 1-9. 
[125] LANG, D. Deep-level transient spectroscopy: A new method to characterize traps in semiconductors. Journal of Applied Physics 45, 7 (1974), 3023-3032.

[126] Laue, E., Skilling, J., And Staunton, J. Maximum entropy reconstruction of spectra containing antiphase peaks. Journal of Magnetic Resonance (1969) 63, 2 (1985), 418-424.

[127] LE VERRIER, U. Sur les variations sculaires des lments des orbites pour les sept plantes principales. Journal de Mathmatiques Pures et Appliques 5, 1 (1840), 220-254.

[128] LEE, H. B. The Cramer-Rao bound on frequency estimates of signals closely spaced in frequency. IEEE Transactions on Signal Processing 40, 6 (1992), 1507-1517.

[129] LEE, T. C., AND NIEDERER, P. Basic engineering for medics and biologists: An ESEM primer, vol. 152. IOS Press, 2010.

[130] Levesque, I. R., Giacomini, P. S., NARAyanan, S., Ribeiro, L. T., Sled, J. G., Arnold, D. L., And Pike, G. B. Quantitative magnetization transfer and myelin water imaging of the evolution of acute multiple sclerosis lesions. Magnetic Resonance in Medicine 63, 3 (2010), 633-640.

[131] Li, D., HANsen, A., Bruschweiler-Li, L., AND BRUschweiler, R. Non-uniform and absolute minimal sampling for highthroughput multidimensional NMR applications. Chemistry-A European Journal (2018).

[132] Li, Y., RAZAVilaR, J., AND LiU, K. R. A high-resolution technique for multidimensional NMR spectroscopy. IEEE Transactions on Biomedical Engineering 45, 1 (1998), 78-86.

[133] LIM, J. S. Two-dimensional signal and image processing. Englewood Cliffs, NJ, Prentice Hall, 1990, 710 p. (1990). 
[134] LiU, J., AND LiU, X. An eigenvector-based approach for multidimensional frequency estimation with improved identifiability. IEEE Transactions on Signal Processing 54, 12 (2006), 4543-4556.

[135] LiU, M., MaO, X.-A., Ye, C., HuAng, H., Nicholson, J. K., And LINDON, J. C. Improved watergate pulse sequences for solvent suppression in NMR spectroscopy. Journal of Magnetic Resonance 132, 1 (1998), 125-129.

[136] LiU, X., AND Sidiropoulos, N. D. On constant modulus multidimensional harmonic retrieval. In Acoustics, Speech, and Signal Processing (ICASSP), 2002 IEEE International Conference on (2002), vol. 3, IEEE, pp. III-2977.

[137] LiU, Z.-S., Li, J., AND StOICA, P. Relax-based estimation of damped sinusoidal signal parameters. Signal processing 62, 3 (1997), 311-321.

[138] Loudon, M., AND PARISE, J. Organic chemistry, roberts and company publishers, 2009.

[139] LudwiG, C., AND Viant, M. R. Two-dimensional J-resolved NMR spectroscopy: review of a key methodology in the metabolomics toolbox. Phytochemical Analysis 21, 1 (2010), 22-32.

[140] Luthon, F., Blanpain, R., Decorps, M., And Albrand, J.-P. Parametric spectrum analysis of 2D NMR signals. application to in vivo J spectroscopy. Journal of Magnetic Resonance (1969) 81, 3 (1989), 538-551.

[141] Madsen, K., Nielsen, H. B., And TinglefF, O. Methods for nonlinear least squares problems.

[142] Mandelshtam, V. A., Taylor, H. S., and Shaka, A. Application of the filter diagonalization method to one-and two- 
dimensional NMR spectra. Journal of Magnetic Resonance 133, 2 (1998), 304-312.

[143] Maranò, S. Array processing for seismic surface waves. PhD thesis, ETH Zurich, 2013.

[144] Margaret Cheng, H.-L., Stikov, N., Ghugre, N. R., And Wright, G. A. Practical medical applications of quantitative MR relaxometry. Journal of Magnetic Resonance Imaging 36, 4 (2012), 805824.

[145] MARION, D. An introduction to biological NMR spectroscopy. Molecular E Cellular Proteomics 12, 11 (2013), 3006-3025.

[146] Marquardt, D. W. An algorithm for least-squares estimation of nonlinear parameters. Journal of the society for Industrial and Applied Mathematics 11, 2 (1963), 431-441.

[147] Marutyan, K. R., Bretthorst, G. L., Goggans, P. M., And CHAN, C.-Y. The Bayesian analysis software developed at Washington university. In Aip Conference Proceedings (2009), vol. 1193, p. 368.

[148] MCNAmeE, J. M., AND PAN, V. Numerical methods for roots of polynomials, vol. 16. Newnes, 2013.

[149] Metzger, G. J., PAtel, M., And Hu, X. Application of genetic algorithms to spectral quantification. Journal of Magnetic Resonance, Series B 110, 3 (1996), 316-320.

[150] Meyer, R., Fisher, M., Nelson, S., AND Brown, T. Evaluation of manual methods for integration of in vivo phosphorus NMR spectra. NMR in Biomedicine 1, 3 (1988), 131-135.

[151] MierisovÁ, Š., AND AlA-KORPElA, M. Mr spectroscopy quantitation: a review of frequency domain methods. NMR in Biomedicine 14, 4 (2001), 247-259. 
[152] Miszalski, B., Shortridge, K., Saunders, W., Parker, Q. A., AND CROOM, S. M. Multi-object spectroscopy field configuration by simulated annealing. Monthly Notices of the Royal Astronomical Society 371, 4 (2006), 1537-1549.

[153] Mlynárik, V., Cudalbu, C., Xin, L., And Gruetter, R. ${ }^{1} \mathrm{H}$ NMR spectroscopy of rat brain in vivo at 14.1 Tesla: improvements in quantification of the neurochemical profile. Journal of Magnetic Resonance 194, 2 (2008), 163-168.

[154] Mobli, M., AND Hoch, J. C. Nonuniform sampling and non-fourier signal processing methods in multidimensional NMR. Progress in nuclear magnetic resonance spectroscopy 83 (2014), 21-41.

[155] Moon, T. K. The expectation-maximization algorithm. IEEE Signal processing magazine 13, 6 (1996), 47-60.

[156] Moudgil, K., RAO, D., AND NARANG, B. Nuclear magnetic resonance and its applications in medicine. The Indian Journal of Pediatrics 52, 3 (1985), 231-241.

[157] Nelson, F., AND WeaVer, H. Nuclear magnetic resonance spectroscopy in superconducting magnetic fields. Science 146, 3641 (1964), 223-232.

[158] Nelson, S. J., AND BROWN, T. R. The accuracy of quantification from 1D-NMR spectra using the piqable algorithm. Journal of Magnetic Resonance (1969) 84, 1 (1989), 95-109.

[159] Nichols, S., SMith, M., AND SAlami, M. Application of ARMA modeling to multicomponent signals. IFAC Proceedings Volumes 18, 5 (1985), 1473-1478. 7th IFAC/IFORS Symposium on Identification and System Parameter Estimation, York, UK, 3-7 July.

[160] Noggle, J. The nuclear Overhauser effect. Elsevier, 2012. 
[161] Odeblad, E., AND LindSTROM, G. Some preliminary observations on the proton magnetic resonance in biologic samples. Acta Radiologica 43, 6 (1955), 469-476.

[162] Osborne, M. Some aspects of nonlinear least squares calculations. Numerical methods for nonlinear optimization (1972).

[163] Oleary, D. P., AND RUST, B. W. Variable projection for nonlinear least squares problems. Computational Optimization and Applications 54, 3 (2013), 579-593.

[164] PaAns, A., VAalburG, W., AND Woldring, M. A comparison of the sensitivity of PET and NMR for in vivo quantitative metabolic imaging. European journal of nuclear medicine 11, 2-3 (1985), 73-75.

[165] Parrish, R. G., Kurland, R. J., Janese, W. W., and Bakay, L. Proton relaxation rates of water in brain and brain tumors. Science 183, 4123 (1974), 438-439.

[166] Poullet, J.-B., Sima, D. M., And Van Huffel, S. MRS signal quantitation: a review of time-and frequency-domain methods. Journal of Magnetic Resonance 195, 2 (2008), 134-144.

[167] Proctor, W., AND YU, F. The dependence of a nuclear magnetic resonance frequency upon chemical compound. Physical Review 77, 5 (1950), 717.

[168] Pukelsheim, F. Optimal design of experiments, vol. 50. SIAM, 1993.

[169] Qian, K., Rodgers, R. P., Hendrickson, C. L., EMmetT, M. R., AND MARShall, A. G. Reading chemical fine print: Resolution and identification of 3000 nitrogen-containing aromatic compounds from a single electrospray ionization Fourier transform ion cyclotron resonance mass spectrum of heavy petroleum crude oil. Energy $\mathcal{E}$ Fuels 15, 2 (2001), 492-498. 
[170] QU, X., GuO, D., CAO, X., CAI, S., AND Chen, Z. Reconstruction of self-sparse 2D NMR spectra from undersampled data in the indirect dimension. Sensors 11, 9 (2011), 8888-8909.

[171] RAO, B. D. Relationship between matrix pencil and state space based harmonic retrieval methods. IEEE Transactions on Acoustics, Speech, and Signal Processing 38, 1 (1990), 177-179.

[172] RAO, B. D., AND ARUN, K. Model based processing of signals: A state space approach. Proceedings of the IEEE 80, 2 (1992), 283-309.

[173] ReDDY, R. Bloch equations. http://www . mmrrcc.upenn.edu/ mediawiki/images/4/4d/Bloch_equations_Feb_2010_ ol.pdf. Accessed: 24-12-2018.

[174] ReID, L. M., O'DONNELL, C. P., AND DowneY, G. Recent technological advances for the determination of food authenticity. Trends in Food Science \& Technology 17, 7 (2006), 344-353.

[175] Reynolds, W. F., Yu, M., EnRiqueZ, R. G., And LeON, I. Investigation of the advantages and limitations of forward linear prediction for processing 2D data sets. Magnetic resonance in chemistry 35, 8 (1997), 505-519.

[176] Richards, S. A., AND Hollerton, J. C. Essential practical NMR for organic chemistry. John Wiley \& Sons, 2010.

[177] Rife, D. C., AND Boorstyn, R. R. Multiple tone parameter estimation from discrete-time observations. Bell System Technical Journal 55, 9 (1976), 1389-1410.

[178] Rodriguez-Castañeda, F., Haberz, P., Leonov, A., AND GrIESINGER, C. Paramagnetic tagging of diamagnetic proteins for solution NMR. Magnetic Resonance in Chemistry 44, S1 (2006). 
[179] RouquetTe, S., AND NAJim, M. Estimation of frequencies and damping factors by two-dimensional ESPRIT type methods. IEEE Transactions on signal processing 49, 1 (2001), 237-245.

[180] Sacchini, J. J., Steedly, W. M., And Moses, R. L. Twodimensional Prony modeling and parameter estimation. IEEE Transactions on signal processing 41, 11 (1993), 3127-3137.

[181] SAhnoun, S., DJermoune, E.-H., And Brie, D. A comparative study of subspace-based methods for 2-D nuclear magnetic resonance spectroscopy signals.

[182] SAndgren, N., SElÉN, Y., STOICA, P., AND Li, J. Parametric methods for frequency-selective MR spectroscopy-a review. Journal of Magnetic Resonance 168, 2 (2004), 259-272.

[183] SAVA, H., AND MCDONNELL, J. Modified forward-backward overdetermined Prony method and its application in modelling heart sounds. IEE Proceedings-Vision, Image and Signal Processing 142, 6 (1995), 375-380.

[184] ScHMIEDER, P. Multidimensional NMR spectroscopy. In Encyclopedic Reference of Genomics and Proteomics in Molecular Medicine. Springer, 2006, pp. 1204-1208.

[185] Schuyler, A. D., Maciejewski, M. W., Stern, A. S., AND $\mathrm{HoCH}, \mathrm{J}$. C. Formalism for hypercomplex multidimensional NMR employing partial-component subsampling. Journal of Magnetic Resonance 227 (2013), 20-24.

[186] SHEN, Y., AND WIN, M. Z. On the accuracy of localization systems using wideband antenna arrays. IEEE Transactions on Communications 58, 1 (2010). 
[187] Sijbers, J., Den DeKKeR, A. J., Verhoye, M., RAMAN, E. R., AND VAN DYCK, D. Optimal estimation of $T_{2}$ maps from magnitude MR images. In Proc. SPIE, Medical Imaging 1998: Image Processing (1998), vol. 3338, International Society for Optics and Photonics, pp. 384391.

[188] SINGH, S. Application of Prony analysis to characterize pulsed corona reactor measurements. PhD thesis, University of Wyoming, 2003.

[189] Slotboom, J., Boesch, C., AND KReIS, R. Versatile frequency domain fitting using time domain models and prior knowledge. Magnetic resonance in medicine 39, 6 (1998), 899-911.

[190] ŠmejKAlOvÁ, D., AND Piccolo, A. Aggregation and disaggregation of humic supramolecular assemblies by NMR diffusion ordered spectroscopy (DOSY-NMR). Environmental Science \& Technology 42, 3 (2007), 699-706.

[191] Smith, M., Nichols, S., HenKelman, R., And Wood, M. Application of autoregressive modelling in magnetic resonance imaging to remove noise and truncation artifacts. Magnetic resonance imaging 4, 3 (1986), 257-261.

[192] So, H.-C., Chan, F. K., Lau, W. H., and Chan, C.-F. An efficient approach for two-dimensional parameter estimation of a single-tone. IEEE Transactions on Signal Processing 58, 4 (2010), 19992009.

[193] Soininen, P., HaArala, J., Vepsëläinen, J., Niemitz, M., AND LAATIKAINEN, R. Strategies for organic impurity quantification by ${ }^{1} \mathrm{H}$ NMR spectroscopy: Constrained total-line-shape fitting. Analytica Chimica Acta 542, 2 (2005), 178-185.

[194] SOURIAU, J.-M. Une méthode pour la dcomposition spectrale et l'inversion des matrices. Comptes Rend. (1948), 1010-1011. 
[195] SPYROS, A., AND DAIS, P. NMR spectroscopy in food analysis. Royal Society of Chemistry, 2012.

[196] Stefan, D., Di Cesare, F., Andrasescu, A., Popa, E., LAZARIEV, A., Vescovo, E., STRbaK, O., Williams, S., STARCUK, Z., CABANAS, M., ET AL. Quantitation of magnetic resonance spectroscopy signals: the jMRUI software package. Measurement Science and Technology 20, 10 (2009), 104035.

[197] STEPHENSON, D. S. Linear prediction and maximum entropy methods in NMR spectroscopy. Progress in Nuclear Magnetic Resonance Spectroscopy 20, 6 (1988), 515-626.

[198] StoicA, P., AND BABU, P. Parameter estimation of exponential signals: A system identification approach. Digital Signal Processing 23, 5 (2013), 1565-1577.

[199] STOICA, P., LI, J., AND SÖDERSTRÖM, T. On the inconsistency of IQML. Signal Processing 56, 2 (1997), 185-190.

[200] StoicA, P., And Marzetta, T. L. Parameter estimation problems with singular information matrices. IEEE Transactions on Signal Processing 49, 1 (2001), 87-90.

[201] StoicA, P., AND Moses, R. L. Spectral analysis of signals, vol. 452. Pearson Prentice Hall Upper Saddle River, NJ, 2005.

[202] STOICA, P., AND SELEN, Y. Model-order selection: a review of information criterion rules. IEEE Signal Processing Magazine 21, 4 (2004), $36-47$.

[203] StoicA, P., And Soderstrom, T. The Steiglitz-McBride identification algorithm revisited-convergence analysis and accuracy aspects. IEEE Transactions on Automatic Control 26, 3 (1981), 712-717. 
[204] Stoica, P., AND Sundin, T. Nonparametric NMR spectroscopy. Journal of Magnetic Resonance 152, 1 (2001), 57-69.

[205] StRANG, G. Introduction to linear algebra. Wellesley-Cambridge Press, 1993.

[206] Strang, G. Computational science and engineering. WellesleyCambridge Press, 2007.

[207] SWINGLER, D. N. Frequency estimation for closely spaced sinsoids: Simple approximations to the Cramer-Rao lower bound. IEEE transactions on signal processing 41, 1 (1993), 489.

[208] SWINGLER, D. N. Approximations to the Cramer-Rao lower bound for a single damped exponential signal. Signal processing 75, 2 (1999), 197-200.

[209] THERRIEn, C. W. Discrete random signals and statistical signal processing. Prentice Hall PTR, 1992.

[210] TiRendi, C. F., AND Martin, J. F. Fast linear prediction processing in two-dimensional NMR spectroscopy. Journal of Magnetic Resonance (1969) 81, 3 (1989), 577-585.

[211] Titterington, D. Optimal design: Some geometrical aspects of D-optimality. Biometrika 62, 2 (1975), 313-320.

[212] TKaCenKo, A., AND VAidyanathan, P. Sinusoidal frequency estimation using filter banks. In Acoustics, Speech, and Signal Processing, 2001. Proceedings.(ICASSP'01). 2001 IEEE International Conference on (2001), vol. 5, IEEE, pp. 3089-3092.

[213] TOMCZAK, M., AND DJERmoune, E.-H. A subband ARMA modeling approach to high-resolution NMR spectroscopy. Journal of Magnetic Resonance 158, 1 (2002), 86-98. 
[214] TomczaK, M., Djermoune, E.-H., And Mutzenhardt, P. HighResolution MR Spectroscopy Via Adaptive Sub-Band Decomposition. In New Research on Magnetic Resonance, B. C. Castleman, Ed. Novascience Publishers, 2007, pp. 241-289.

[215] Trudnowski, D. J., Johnson, J., And Hauer, J. F. Making Prony analysis more accurate using multiple signals. IEEE Transactions on Power Systems 14, 1 (1999), 226-231.

[216] UMESH, S., AND TUFTS, D. W. Estimation of parameters of exponentially damped sinusoids using fast maximum likelihood estimation with application to NMR spectroscopy data. IEEE Transactions on Signal Processing 44, 9 (1996), 2245-2259.

[217] VAlLiSNERI, M. Use and abuse of the Fisher information matrix in the assessment of gravitational-wave parameter-estimation prospects. Phys. Rev. D 77 (Feb 2008), 042001.

[218] Van den BoogaArt, A., Ala-Korpela, M., Howe, F. A., RoDrigues, L. M., StubBs, M., AND GRIfFiths, J. R. Magnetic resonance spectroscopy data analysis: time or frequency domain? Magnetic Resonance Materials in Physics, Biology and Medicine 2, 3 (1994), $479-482$.

[219] Van den BoogaArt, A., Ala-Korpela, M., JokisAari, J., AND GRIFFITHS, J. Time and frequency domain analysis of NMR data compared: an application to 1D 1H spectra of lipoproteins. Magnetic resonance in medicine 31, 4 (1994), 347-358.

[220] VAN HufFeL, S. Enhanced resolution based on minimum variance estimation and exponential data modeling. Signal processing 33, 3 (1993), 333-355. 
[221] Vanhamme, L., Sundin, T., Hecke, P. V., And Huffel, S. V. MR spectroscopy quantitation: a review of time-domain methods. NMR in Biomedicine 14, 4 (2001), 233-246.

[222] Vanhamme, L., Van Den Boogaart, A., AND Van Huffel, S. Improved method for accurate and efficient quantification of MRS data with use of prior knowledge. Journal of Magnetic Resonance 129, 1 (1997), 35-43.

[223] Vanhamme, L., Van Huffel, S., Van Hecke, P., And Van ORMONDT, D. Time-domain quantification of series of biomedical magnetic resonance spectroscopy signals. Journal of Magnetic Resonance 140, 1 (1999), 120-130.

[224] Vanhuffel, S., Chen, H., Decanniere, C., And Vanhecke, P. Algorithm for time-domain NMR data fitting based on total least squares. Journal of Magnetic Resonance, Series A 110, 2 (1994), 228237.

[225] WAgNeR, G. An account of nmr in structural biology. Nature Structural Biology 4 (1997), 841-844.

[226] WALKER, J. S. A primer on wavelets and their scientific applications. CRC press, 2002.

[227] Wang, X., Aboutanios, E., And Amin, M. G. Adaptive array thinning for enhanced DOA estimation. IEEE Signal Processing Letters 22, 7 (2015), 799-803.

[228] WANG, Y.-M., LeE, H., AND APTE, D. V. Quantitative NMR spectroscopy by matrix pencil methods. International Journal of Imaging Systems and Technology 4, 3 (1992), 201-206.

[229] Weljie, A. M., Newton, J., Mercier, P., Carlson, E., AND SLUPSKY, C. M. Targeted profiling: quantitative analysis of ${ }^{1} \mathrm{H}$ NMR metabolomics data. Analytical Chemistry 78, 13 (2006), 4430-4442. 
[230] Westrhal, F., Junge, T., Girreser, U., Greibl, W., AND DoerING, C. Mass, NMR and IR spectroscopic characterization of pentedrone and pentylone and identification of their isocathinone byproducts. Forensic science international 217, 1 (2012), 157-167.

[231] Whittall, K. P., MacKay, A. L., And Li, D. K. Are monoexponential fits to a few echoes sufficient to determine $T_{2}$ relaxation for in vivo human brain? Magnetic Resonance in Medicine 41, 6 (1999), 1255-1257.

[232] Wigren, T., AND NeHORAi, A. Asymptotic Cramer-Rao bounds for estimation of the parameters of damped sine waves in noise. IEEE Transactions on signal processing 39, 4 (1991), 1017-1020.

[233] Williamson, M. P., Havel, T. F., And WÜthrich, K. Solution conformation of proteinase inhibitor IIA from bull seminal plasma by ${ }^{1} \mathrm{H}$ nuclear magnetic resonance and distance geometry. Journal of molecular biology 182, 2 (1985), 295-315.

[234] Wilson, A. G., Wu, Y., Holland, D. J., Nowozin, S., ManTLE, M. D., GLADDEN, L. F., AND BlAKE, A. Bayesian inference for $\mathrm{nmr}$ spectroscopy with applications to chemical quantification. arXiv preprint arXiv:1402.3580 (2014).

[235] Wu, Y., Holland, D. J., Mantle, M. D., Wilson, A. G., NowozIN, S., BlAKE, A., AND GladDen, L. F. A Bayesian method to quantifying chemical composition using NMR: Application to porous media systems. In 2014 22nd European Signal Processing Conference (EUSIPCO) (2014), IEEE, pp. 2515-2519.

[236] WÜTHRICH, K. Protein structure determination in solution by NMR spectroscopy. Journal of Biological Chemistry 265, 36 (1990), 2205922062. 
[237] XI, Y., AND ROCKE, D. M. Baseline correction for NMR spectroscopic metabolomics data analysis. BMC bioinformatics 9, 1 (2008), 324.

[238] XIONG, J., LIU, K., DA Costa, J. P. C., AND WANG, W.-Q. Bayesian information criterion for multidimensional sinusoidal order selection. In Acoustics, Speech and Signal Processing (ICASSP), 2017 IEEE International Conference on (2017), IEEE, pp. 3106-3110.

[239] YAO, Y.-X., AND PANDIT, S. M. Cramer-Rao lower bounds for a damped sinusoidal process. IEEE Transactions on Signal Processing 43, 4 (1995), 878-885.

[240] YE, ShangLin, E. E. . T. F. O. E. U. Signal detection and parameter estimation for nuclear magnetic resonance spectroscopy, 2015.

[241] Ye, S., AND AbOUtANiOS, E. Efficient peak extraction of proton NMR spectroscopy using lineshape adaptation. In 2014 IEEE International Conference on Acoustics, Speech and Signal Processing (ICASSP) (2014), IEEE, pp. 5661-5665.

[242] ZAIBAQ, N., AND BARRON, A. Two-dimensional NMR experiments. https://cnx.org/exports/ 62103fd7-1b04-45f4-8b3f-b0a821029322@2.pdf/ two-dimensional-nmr-experiments-2.pdf.

Accessed: 28-12-2018.

[243] Zeng, Y., TANG, J., Bush, C. A., AND Norris, J. R. Enhanced spectral resolution in 2D NMR signal analysis using linear prediction extrapolation and apodization. Journal of Magnetic Resonance (1969) 83, 3 (1989), 473-483. 